\title{
Stability of a discrete-time general delayed viral model with antibody and cell-mediated immune responses
}

\author{
A.M. Elaiw ${ }^{1 *}$ (D) and M.A. Alshaikh ${ }^{1,2}$
}

\section{"Correspondence:}

a_m_elaiw@yahoo.com

'Department of Mathematics,

Faculty of Science, King Abdulaziz

University, Jeddah, Saudi Arabia

Full list of author information is

available at the end of the article

\section{Springer}

\begin{abstract}
We propose a discrete-time viral model with antibody and cell-mediated immune responses. Two types of infected cells are incorporated into the model, namely latently infected and actively infected. The incidence rate of infection as well as the production and removal rates of all compartments are modeled by general nonlinear functions. The model contains three types of intracellular time delays. We utilize nonstandard finite difference (NSFD) method to discretize the continuous-time model. We prove that NSFD preserves the positivity and boundedness of the solutions of the model. Based on four threshold parameters, the existence of the five equilibria of the model is established. We perform global stability of all equilibria of the model by using Lyapunov approach. Numerical simulations are carried out to illustrate our theoretical results. The impact of time delay on the viral dynamics is established.
\end{abstract}

Keywords: Viral dynamics; Latency; Time delay; Global stability; Antibody; Cell-mediated response; Discrete-time model; Lyapunov function

\section{Introduction}

Mathematical modeling and analysis of within host human viral infections have provided useful insights into the understanding of interplay between three main compartments: viruses, target cells, and infected cells. Nowak and Bangham [1] have proposed the basic virus infection model in the form

$$
\left\{\begin{array}{l}
\dot{F}(t)=\delta-\beta F(t)-\kappa F(t) H(t) \\
\dot{S}(t)=\kappa F(t) H(t)-a S(t) \\
\dot{H}(t)=\theta S(t)-c H(t)
\end{array}\right.
$$

where $F, S$, and $H$ are the healthy (uninfected) target cells, actively infected cells, and free virus particles. $\delta$ and $\beta F$ are the production and natural death rates of the healthy cells, respectively. The incidence rate is modeled by $\kappa F H$. The death rate of actively infected cells is given by $a S$. The free viruses are generated at rate $\theta S$ and cleared at rate $c H$. The model has been developed to describe within host dynamics of different viruses such as

(c) The Author(s) 2020. This article is licensed under a Creative Commons Attribution 4.0 International License, which permits use, sharing, adaptation, distribution and reproduction in any medium or format, as long as you give appropriate credit to the original author(s) and the source, provide a link to the Creative Commons licence, and indicate if changes were made. The images or other third party material in this article are included in the article's Creative Commons licence, unless indicated otherwise in a credit line to the material. If material is not included in the article's Creative Commons licence and your intended use is not permitted by statutory regulation or exceeds the permitted use, you will need to obtain permission directly from the copyright holder. To view a copy of this licence, visit http://creativecommons.org/licenses/by/4.0/. 
human immunodeficiency virus (HIV) [2, 3], hepatitis C virus (HCV) $[4,5]$, hepatitis B virus (HBV) [6, 7], chikungunya virus (CHIKV) [8], and dengue virus [9].

The immune system works to defend the body against attacks by foreign invaders. $B$ cells and CTL cells play a central role in the specific immune response. In general, B cells produce antibodies that neutralize the viruses, namely antibody immune response and CTL cells attack and kill virus-infected cells, namely cell-mediated immune response. The impact of cell-mediated immune response on the virus dynamics has been modeled [1] as follows:

$$
\left\{\begin{array}{l}
\dot{F}(t)=\delta-\beta F(t)-\kappa F(t) H(t), \\
\dot{S}(t)=\kappa F(t) H(t)-a S(t)-\lambda S(t) Z(t), \\
\dot{H}(t)=\theta S(t)-c H(t), \\
\dot{Z}(t)=g S(t) Z(t)-\xi Z(t),
\end{array}\right.
$$

where $Z$ is the concentration of the CTL cells. The CTL cells are proliferated at rate $g S Z$, die at rate $\xi Z$, and kill infected cells at rate $\lambda S Z$. Model (2) has been extended in several works (see, e.g., [10-13]). In other words, the basic model (1) has been modified to take into account the effect of antibody immune response in [14] as follows:

$$
\left\{\begin{array}{l}
\dot{F}(t)=\delta-\beta F(t)-\kappa F(t) H(t), \\
\dot{S}(t)=\kappa F(t) H(t)-a S(t), \\
\dot{H}(t)=\theta S(t)-c H(t)-d H(t) Y(t), \\
\dot{Y}(t)=q H(t) Y(t)-\eta Y(t),
\end{array}\right.
$$

where $Y$ is the concentration of antibodies. The free viruses are removed by the antibodies at rate $d H Y$. The antibodies are proliferated at rate $q H Y$ and die at rate $\eta Y$. In the literature, the effect of antibody immune response has been incorporated into mathematical models of various virus infections (see, e.g., [15-17]).

In models (1)-(3) it has been assumed that once a healthy cell is contacted by a virus it becomes productive instantaneously. In fact, a number of intracellular processes is needed to produce new viruses. In case of HIV infection the intracellular processes take approximately 0.9 days [18]. Therefore, the intracellular time delay has a significant effect on the virus dynamics. Delayed viral infection models have been constructed and analyzed in several works (see, e.g., [19-32]). To incorporate both cell-mediated and antibody immune responses as well as the time delay into the virus dynamics, Wodarz [33] has proposed the following model:

$$
\left\{\begin{array}{l}
\dot{F}(t)=\delta-\beta F(t)-\kappa F(t) H(t), \\
\dot{S}(t)=\kappa e^{-\mu \tau} F(t-\tau) H(t-\tau)-a S(t)-\lambda S(t) Z(t), \\
\dot{H}(t)=\theta S(t)-c H(t)-d H(t) Y(t), \\
\dot{Y}(t)=q H(t) Y(t)-\eta Y(t), \\
\dot{Z}(t)=g S(t) Z(t)-\xi Z(t),
\end{array}\right.
$$

where, $e^{-\mu \tau}$ represents the survival rate of infected cells after the interval $\tau$, and $\mu$ is a positive constant. Model (4) has been extended in [34-36]. It has been reported in [37, 38] 
that in case of HIV infection, latent HIV reservoirs serve as a major obstruction in treating HIV infection. Latently infected cells have been incorporated into the virus dynamics model with both cell-mediated and antibody in [25] and [39]. The bilinear incidence rate associated with the mass action principle can be insufficient to describe infection process in detail. The most obvious reason for a nonlinear incidence rate is that the number of free pathogen particles can vary in a very wide range, from a few particles up to hundreds of millions of them in the case of virus. The bilinear interaction term, which may be considered as an approximation, is hardly able to adequately describe the process that runs over such a range of variables. For instance, if the number of free pathogen particles is very high, so that exposure of a susceptible host is virtually certain, then the incidence rate may respond more slowly than linear to the further increase in the number of the pathogen particles [40].

In all the above mentioned works the viral infection is modeled by a system of ordinary or delay differential equations. These models are nonlinear, and calculating the exact analytical solution is difficult or impossible. Therefore, only approximate discrete-time models can be obtained by using suitable numerical approximation methods. One of the discretization methods which has been widely used to discretize viral infection models is called nonstandard finite difference (NSFD) [41]. It has been established that NSFD has the advantage of preserving the essential qualitative features of these models such as equilibria, positivity, boundedness, and global behaviors of solutions independently of the chosen step-size [42-46]. The impact of cell-mediated immune response has been incorporated into the discrete-time virus dynamics models in [47, 48]. In very recent works, Elaiw and Alshaikh [49-51] have proposed and investigated a class of discrete-time virus infection models with antibody immune response. However, the impact of both antibody and cell-mediated immune responses on the discrete-time virus infection model has not been investigated before.

The aim of the present paper is to formulate and analyze a discrete-time viral infection model with both antibody and cell-mediated immune responses. The model considers both latently infected cells and actively infected cells. The incidence rate of infection as well as the production and removal rates of all compartments are modeled by general nonlinear functions. We discretize the continuous-time model by using NSFD method. We first show that the solutions of the discrete-time model are positive and bounded, then we prove the global stability of the equilibria by constructing Lyapunov functions. Moreover, we perform numerical simulations to support the global stability results.

The achievements in this present paper look ahead to research perspectives focused on pattern formation induced by the action of the external environments, for instance, by Keller Segel dynamics [52]. The present literature is focused simply on the original SIR model [52], while it appears interesting extending the qualitative and computational analysis to more advanced models such as the one treated in our paper.

\section{The model}

We introduce the following general viral infection model with three types of time delays and both antibody and cell-mediated immune responses:

$$
\begin{aligned}
& \dot{F}(t)=\Theta(F(t))-\Lambda(F(t), H(t)), \\
& \dot{K}(t)=(1-\varepsilon) e^{-\mu_{1} \tau_{1}} \Lambda\left(F\left(t-\tau_{1}\right), H\left(t-\tau_{1}\right)\right)-(\alpha+m) \digamma_{1}(K(t)),
\end{aligned}
$$




$$
\begin{aligned}
\dot{S}(t)= & \varepsilon e^{-\mu_{2} \tau_{2}} \Lambda\left(F\left(t-\tau_{2}\right), H\left(t-\tau_{2}\right)\right)+m \digamma_{1}(K(t))-a \digamma_{2}(S(t)) \\
& -\lambda \digamma_{2}(S(t)) \digamma_{5}(Z(t)), \\
\dot{H}(t)= & \theta e^{-\mu_{3} \tau_{3}} \digamma_{2}\left(S\left(t-\tau_{3}\right)\right)-c \digamma_{3}(H(t))-d \digamma_{3}(H(t)) \digamma_{4}(Y(t)), \\
\dot{Y}(t)= & q \digamma_{3}(H(t)) \digamma_{4}(Y(t))-\eta \digamma_{4}(Y(t)), \\
\dot{Z}(t)= & g \digamma_{2}(S(t)) \digamma_{5}(Z(t))-\xi \digamma_{5}(Z(t)),
\end{aligned}
$$

where $K$ is the concentration of latently infected cells. The parameter $\varepsilon$, with $0<\varepsilon<1$, is a fraction of the healthy cells that become latently infected. The terms $\alpha \digamma_{1}(K)$ and $m \digamma_{1}(K)$ represent the death and activation rates of the latently infected cells. We suppose that the viruses contact the healthy cells at times $t-\tau_{1}$ and $t-\tau_{2}$, respectively, the cells become latently infected and actively infected at time $t$, where $\tau_{1}$ and $\tau_{2}$ are positive constants. The immature viruses at time $t-\tau_{3}$ are assumed to be mature at time $t$, where $\tau_{3}$ is a positive constant. $e^{-\mu_{j} \tau_{j}}, j=1,2,3$, is the probability of the cells and viruses survival during the delay periods, where $\mu_{1}, \mu_{2}$, and, $\mu_{3}$ are positive constants. Here $\Theta, \Lambda$, and $\digamma_{i}, i=1, \ldots, 5$, are general nonlinear functions satisfy the following conditions:

C1 (i) There exists $F^{0}>0$ such that $\Theta\left(F^{0}\right)=0, \Theta(F)>0$ for $F \in\left[0, F^{0}\right)$;

(ii) $\Theta^{\prime}(F)<0$ for all $F>0$;

(iii) $\exists b, \bar{b}>0$ such that $\Theta(F) \leq b-\bar{b} F$ for all $F \geq 0$.

Here, $F^{0}$ is the equilibrium susceptible cell concentration in the absence of viral infection. Condition $\mathrm{C} 1$ implies that $F(t) \rightarrow F^{0}$ as $t \rightarrow \infty$ in the absence of the infection.

$\mathrm{C} 2 \quad$ (i) $\Lambda(F, H)>0$, and $\Lambda(0, H)=\Lambda(F, 0)=0$ for all $F>0, H>0$;

(ii) $\frac{\partial \Lambda(F, H)}{\partial F}>0, \frac{\partial \Lambda(F, H)}{\partial H}>0, \frac{\partial \Lambda(F, 0)}{\partial H}>0$ for all $F>0, H>0$;

(iii) $\frac{d}{d F}\left(\frac{\partial \Lambda(F, 0)}{\partial H}\right)>0$ for all $F>0$.

Furthermore, $\mathrm{C} 2(\mathrm{i})$ means there are no incidences if there are no susceptible cells or free virus particles. For $\mathrm{C} 2$ (ii), the number of new cases monotonically grows with growth in the numbers of susceptible cells and free pathogen particles. Moreover, condition C2(iii) accounts that the infection rate starts growing even if the number of pathogens is very small.

C3 (i) $\digamma_{j}(\rho)>0$ for $\rho>0, \digamma_{j}(0)=0, j=1, \ldots, 5$;

(ii) $\digamma_{j}^{\prime}(\rho)>0$ for $\rho>0, j=1,2,4,5$ and $\digamma_{3}^{\prime}(\rho)>0$ for $\rho \geq 0$;

(iii) There are $v_{j}>0, j=1, \ldots, 5$, such that $\digamma_{j}(\rho) \geq v_{j} \rho$ for $\rho \geq 0$.

Condition $\mathrm{C} 3$ indicates that the natural mortality rates of the infected cells, pathogens, CTL, and antibodies monotonically grow with growth in their populations.

C4 $\frac{\partial}{\partial H}\left(\frac{\Lambda(F, H)}{\digamma_{3}(H)}\right) \leq 0$ for all $H>0$.

The quantity $\frac{\Lambda(F, H)}{\digamma_{3}(H)}$ may be interpreted as the efficiency of the pathogen, that is, the ratio of its infectivity to its removal. Condition C4 mentions that the efficiency of the pathogen is nonincreasing with respect to the population of the pathogens [39].

Remark 1 There are several forms of the general functions which can satisfy $\mathrm{C} 1-\mathrm{C} 4$ such as:

(i) Intrinsic growth rate function $\Theta(F)$ : linear form $\Theta(F)=\delta-\beta F$ [1] and logistic growth form $\Theta(F)=\delta-\beta F+r F\left(1-\frac{F}{F_{\max }}\right)$, where $r<\beta[53,54]$.

(ii) Incidence rate function $\Lambda(F, H)$ : bilinear incidence $\kappa F H$ [55], saturated incidence $\frac{\kappa F H}{1+u H}$ [56], (iii) Holling-type II incidence $\frac{\kappa F H}{1+w F}$ [57], Beddington-DeAngelis 
incidence $\frac{\kappa F H}{1+u H+w F}$ [58], Crowley-Martin incidence $\frac{\kappa F H}{(1+u H)(1+w F)}$ [59], Hill-type incidence $\frac{\kappa F^{\ell} H}{\zeta^{\ell}+F^{\ell}}$ [60], where $\kappa, u, w, \zeta$, and $\ell$ are positive constants.

(iii) Function $\digamma_{i}(\rho)$ : linear $\digamma_{i}(\rho)=v_{i} \rho$ [1] and quadratic $\digamma_{i}(\rho)=v_{i} \rho+\bar{v}_{i} \rho^{2}$ [15], where $v_{i}$ and $\bar{v}_{i}$ are positive constants.

We use the NSFD method [41] to discretize model (5)-(10). Let $t_{n}=n h$, where $h>0$ is the time step size and $n \in \mathbb{N}=\{0,1,2, \ldots\}$. Let $\left(F_{n}, K_{n}, S_{n}, H_{n}, Y_{n}, Z_{n}\right)$ be the approximations of the solution $\left(F\left(t_{n}\right), K\left(t_{n}\right), S\left(t_{n}\right), H\left(t_{n}\right), Y\left(t_{n}\right), Z\left(t_{n}\right)\right)$ of system (5)-(10) at the discrete time points $t_{n}$. Assume that there exist integers $m_{i} \in \mathbb{N}, i=1,2,3$, with $\tau_{i}=h m_{i}$.

$$
\begin{aligned}
\frac{F_{n+1}-F_{n}}{\phi(h)}= & \Theta\left(F_{n+1}\right)-\Lambda\left(F_{n+1}, H_{n}\right), \\
\frac{K_{n+1}-K_{n}}{\phi(h)}= & (1-\varepsilon) e^{-\mu_{1} \tau_{1}} \Lambda\left(F_{n-m_{1}+1}, H_{n-m_{1}}\right)-(\alpha+m) \digamma_{1}\left(K_{n+1}\right), \\
\frac{S_{n+1}-S_{n}}{\phi(h)}= & \varepsilon e^{-\mu_{2} \tau_{2}} \Lambda\left(\digamma_{n-m_{2}+1}, H_{n-m_{2}}\right)+m \digamma_{1}\left(K_{n+1}\right)-a \digamma_{2}\left(S_{n+1}\right) \\
& -\lambda \digamma_{2}\left(S_{n+1}\right) \digamma_{5}\left(Z_{n+1}\right), \\
\frac{H_{n+1}-H_{n}}{\phi(h)}= & \theta e^{-\mu_{3} \tau_{3}} \digamma_{2}\left(S_{n-m_{3}+1}\right)-c \digamma_{3}\left(H_{n+1}\right)-d \digamma_{3}\left(H_{n+1}\right) \digamma_{4}\left(Y_{n+1}\right), \\
\frac{Y_{n+1}-Y_{n}}{\phi(h)}= & q \digamma_{3}\left(H_{n+1}\right) \digamma_{4}\left(Y_{n+1}\right)-\eta \digamma_{4}\left(Y_{n+1}\right), \\
\frac{Z_{n+1}-Z_{n}}{\phi(h)} & =g \digamma_{2}\left(S_{n+1}\right) \digamma_{5}\left(Z_{n+1}\right)-\xi \digamma_{5}\left(Z_{n+1}\right) .
\end{aligned}
$$

The function $\phi(h)$ is a denominator function $[61,62]$ where $\phi(h)=h+O\left(h^{2}\right)$.

The initial conditions of system (11)-(16) are

$$
\begin{aligned}
& F_{\omega}=\psi_{\omega}^{1} \geq 0, \quad K_{\omega}=\psi_{\omega}^{2} \geq 0, \quad S_{\omega}=\psi_{\omega}^{3} \geq 0, \\
& H_{\omega}=\psi_{\omega}^{4} \geq 0, \quad Y_{\omega}=\psi_{\omega}^{5} \geq 0, \quad Z_{\omega}=\psi_{\omega}^{6} \geq 0
\end{aligned}
$$

for all $\omega=-\bar{m},-\bar{m}+1, \ldots, 0$,

$$
\psi_{0}^{i}>0, \quad i=1, \ldots, 6,
$$

where $\bar{m}=\max \left\{m_{1}, m_{2}, m_{3}\right\}$.

\subsection{Preliminaries}

We define a compact set

$$
\Gamma=\left\{(F, K, S, H, Y, Z): 0<F, K, S<\vartheta_{1}, 0<H<\vartheta_{3}, 0<Y<\vartheta_{4}, 0<Z<\vartheta_{2}\right\},
$$

where $\vartheta_{1}=\frac{b}{\sigma_{1}}, \vartheta_{2}=\frac{g \vartheta_{1}}{\lambda}, \vartheta_{3}=\frac{\theta \vartheta_{1}}{\sigma_{2}}, \vartheta_{4}=\frac{q \theta \vartheta_{1}}{d \sigma_{2}}, \sigma_{1}=\min \left\{\bar{b}, \alpha v_{1}, a, \xi\right\}$, and $\sigma_{2}=\min \{c, \eta\}$.

Lemma 1 Suppose that Conditions C1-C3 are satisfied and $\digamma_{j}(\rho)=\rho, j=2,3,4,5$, then any solution $\left(F_{n}, K_{n}, S_{n}, H_{n}, Y_{n}, Z_{n}\right)$ of model (11)-(16) with initial conditions (17) is positive and ultimately bounded. 
Lemma 2 For system (11)-(16), let Conditions C1-C4 hold true, then there exist four threshold parameters $\mathcal{R}_{0}>0, \mathcal{R}_{1}^{Y}>0, \mathcal{R}_{1}^{Z}>0$, and $\mathcal{R}_{2}^{Z}>0$ such that

(i) if $\mathcal{R}_{0} \leq 1$, then the system has only one equilibrium $Q^{0}$.

(ii) if $\mathcal{R}_{1}^{Y} \leq 1<\mathcal{R}_{0}$ and $\mathcal{R}_{1}^{Z} \leq 1<\mathcal{R}_{0}$, then the system has two equilibria $Q^{0}$ and $Q^{*}$.

(iii) if $\mathcal{R}_{1}^{Y}>1$ and $\mathcal{R}_{2}^{Z} \leq 1$, then the system has three equilibria $Q^{0}, Q^{*}$, and $\bar{Q}$.

(iv) if $\mathcal{R}_{1}^{Z}>1$ and $\mathcal{R}_{1}^{Y} / \mathcal{R}_{2}^{Z} \leq 1$, then the system has three equilibria $Q^{0}, Q^{*}$, and $\widehat{Q}$.

(v) if $\mathcal{R}_{1}^{Y}>\mathcal{R}_{2}^{Z}>1$, then the system has five equilibria $Q^{0}, Q^{*}, \bar{Q}, \widehat{Q}$, and $\widetilde{Q}$.

The proofs of Lemmas 1-2 are given in Appendix.

\subsection{Global stability}

We define the function

$$
G(\rho)=\rho-\ln \rho-1 .
$$

Clearly, $G(\rho) \geq 0$ and $G(1)=0$ for all $\rho>0$, therefore

$$
\ln \rho \leq \rho-1
$$

Theorem 1 Suppose that Conditions C1-C4 are satisfied and $\mathcal{R}_{0} \leq 1$, then $Q^{0}$ of model (11)-(16) is G.A.S.

Remark 2 Conditions C2-C4 imply that

$$
\left(\Lambda(F, H)-\Lambda\left(F, H^{*}\right)\right)\left(\frac{\Lambda(F, H)}{\digamma_{3}(H)}-\frac{\Lambda\left(F, H^{*}\right)}{\digamma_{3}\left(H^{*}\right)}\right) \leq 0,
$$

which yields

$$
\left(1-\frac{\Lambda\left(F, H^{*}\right)}{\Lambda(F, H)}\right)\left(\frac{\Lambda(F, H)}{\Lambda\left(F, H^{*}\right)}-\frac{\digamma_{3}(H)}{\digamma_{3}\left(H^{*}\right)}\right) \leq 0 .
$$

Lemma 3 Suppose that Conditions C1-C4 are satisfied and $\mathcal{R}_{0}>1$, then

(i) $\operatorname{sgn}\left(\bar{F}-F^{*}\right)=\operatorname{sgn}\left(H^{*}-\bar{H}\right)=\operatorname{sgn}\left(\mathcal{R}_{1}^{Y}-1\right)$,

(ii) $\operatorname{sgn}\left(\widehat{F}-F^{*}\right)=\operatorname{sgn}\left(H^{*}-\widehat{H}\right)=\operatorname{sgn}\left(S^{*}-\widehat{S}\right)=\operatorname{sgn}\left(\mathcal{R}_{1}^{Z}-1\right)$.

Theorem 2 Suppose that Conditions C1-C4 are satisfied, $\mathcal{R}_{1}^{Y} \leq 1<\mathcal{R}_{0}$ and $\mathcal{R}_{1}^{Z} \leq 1<\mathcal{R}_{0}$, then $Q^{*}$ of system (11)-(16) is G.A.S.

Theorem 3 If Conditions C1-C4 hold, $\mathcal{R}_{1}^{Y}>1$ and $\mathcal{R}_{2}^{Z} \leq 1$, then $\bar{Q}$ of system (11)-(16) is G.A.S.

Theorem 4 If Conditions $\mathrm{C} 1-\mathrm{C} 4$ are satisfied, $\mathcal{R}_{1}^{Z}>1$ and $\mathcal{R}_{1}^{Y} / \mathcal{R}_{2}^{Z} \leq 1$, then $\widehat{Q}$ of system (11)-(16) is G.A.S.

Theorem 5 If Conditions C1-C4 are satisfied and $\mathcal{R}_{1}^{Y}>\mathcal{R}_{2}^{Z}>1$, then $\widetilde{Q}$ of system (11)(16) is G.A.S.

The proofs of Theorems $1-5$ and Lemma 3 are given in Appendix. 


\section{Numerical simulations}

We perform our simulation by choosing

$$
\begin{aligned}
& \Theta(F)=\delta-\beta F+r F\left(1-\frac{F}{F_{\max }}\right), \quad \Lambda(F, H)=\frac{\kappa F H}{1+c_{1} H} \\
& \digamma_{i}(\rho)=\rho, \quad i=1, \ldots, 5
\end{aligned}
$$

where $r>0$ is the maximum proliferation rate of the healthy cells and $F_{\max }>0$ is the maximum level of healthy cells concentration in the body. If $F$ reaches $F_{\max }$, it should decrease. Therefore, system (11)-(16) becomes

$$
\begin{aligned}
& \frac{F_{n+1}-F_{n}}{\phi(h)}=\delta-\beta F_{n+1}+r F_{n+1}\left(1-\frac{F_{n+1}}{F_{\max }}\right)-\frac{\kappa F_{n+1} H_{n}}{1+c_{1} H_{n}}, \\
& \frac{K_{n+1}-K_{n}}{\phi(h)}=\frac{(1-\varepsilon) e^{-\mu_{1} \tau_{1}} \kappa F_{n-m_{1}+1} H_{n-m_{1}}}{1+c_{1} H_{n-m_{1}}}-(\alpha+m) K_{n+1}, \\
& \frac{S_{n+1}-S_{n}}{\phi(h)}=\frac{\varepsilon e^{-\mu_{2} \tau_{2}} \kappa F_{n-m_{2}+1} H_{n-m_{2}}}{1+c_{1} H_{n-m_{2}}}+m K_{n+1}-a S_{n+1}-\lambda S_{n+1} Z_{n+1}, \\
& \frac{H_{n+1}-H_{n}}{\phi(h)}=\theta e^{-\mu_{3} \tau_{3}} S_{n-m_{3}+1}-c H_{n+1}-d H_{n+1} Y_{n+1}, \\
& \frac{Y_{n+1}-Y_{n}}{\phi(h)}=q H_{n+1} Y_{n+1}-\eta Y_{n+1}, \\
& \frac{Z_{n+1}-Z_{n}}{\phi(h)}=g S_{n+1} Z_{n+1}-\xi Z_{n+1} .
\end{aligned}
$$

The denominator function $\phi(h)$ can take the form $[61,62]$

$$
\phi(h)=\frac{1-e^{-\beta h}}{\beta} .
$$

We assume that $r<\beta$ [54]. Now we check the validity of Conditions $\mathrm{C} 1-\mathrm{C} 4$ for the functions given by (20). We have $\Theta(0)=\delta>0, \Theta\left(F^{0}\right)=0$, where

$$
F^{0}=\frac{F_{\max }}{2 r}\left(r-\beta+\sqrt{(r-\beta)^{2}+\frac{4 r \delta}{F_{\max }}}\right) .
$$

Since $r<\beta$, then

$$
\Theta^{\prime}(F)=-\beta+r-\frac{2 r F}{F_{\max }}<0 .
$$

It follows that $\Theta(F)>0$ for all $F \in\left[0, F^{0}\right)$ and

$$
\Theta(F)=\delta-(\beta-r) F-r \frac{F^{2}}{F_{\max }}<\delta-(\beta-r) F .
$$


Let $b=\delta>0$ and $\bar{b}=\beta-r>0$. Thus, C1 is satisfied. We have also

$$
\begin{aligned}
& \Lambda(F, H)=\frac{\kappa F H}{1+c_{1} H}>0 \text { and } \Lambda(0, H)=\Lambda(F, 0)=0 \text { for all } F>0, H>0, \\
& \frac{\partial \Lambda(F, H)}{\partial F}=\frac{\kappa H}{1+c_{1} H}>0 \text { for all } F>0 \text { and } H>0, \\
& \frac{\partial \Lambda(F, H)}{\partial H}=\frac{\kappa F}{\left(1+c_{1} H\right)^{2}}>0 \text { for all } F>0 \text { and } H>0, \\
& \frac{\partial \Lambda(F, 0)}{\partial H}=\kappa F>0 \quad \text { for all } F>0, \\
& \frac{d}{d F}\left(\frac{\partial \Lambda(F, 0)}{\partial H}\right)=\kappa>0 \text { for all } F>0 .
\end{aligned}
$$

Therefore, Condition C2 is satisfied. We have $\digamma_{j}(\rho)=\rho>0$ for all $\rho>0$ and $\digamma_{j}(0)=0$, $j=1, \ldots, 5$, and $\digamma_{j}^{\prime}(\rho)=1>0, j=1, \ldots, 5$, for all $\rho \geq 0$. Then Condition C3 is satisfied, where $v_{j}=1, j=1, \ldots, 5$. Finally, we have

$$
\frac{\partial}{\partial H}\left(\frac{\Lambda(F, H)}{\digamma_{3}(H)}\right)=\frac{-\kappa F c_{1}}{\left(1+c_{1} H\right)^{2}}<0 \quad \text { for all } F>0 \text { and } H>0 \text {. }
$$

Therefore, Condition C4 holds true and hence Theorems 1-5 are applicable.

For this system, the threshold parameters are given by

$$
\begin{aligned}
& \mathcal{R}_{0}=\frac{\theta \gamma \kappa F^{0}}{a c}, \quad \mathcal{R}_{1}^{Y}=\frac{\theta \gamma \kappa \bar{F}}{a c\left(1+c_{1} \bar{H}\right)}, \\
& \mathcal{R}_{1}^{Z}=\frac{\theta \gamma \kappa \widehat{F}}{a c\left(1+c_{1} \widehat{H}\right)}, \quad \mathcal{R}_{2}^{Z}=\frac{e^{\mu_{3} \tau_{3}} \gamma \kappa \widetilde{F} \widetilde{H}}{a\left(1+c_{1} \widetilde{H}\right) \widetilde{S}} \\
& \mathcal{R}_{1}^{Y} \backslash \mathcal{R}_{2}^{Z}=\frac{\theta e^{-\mu_{3} \tau_{3}} \xi g}{c g \eta},
\end{aligned}
$$

where

$$
\begin{aligned}
& \bar{F}=\frac{-\bar{B}+\sqrt{\bar{B}^{2}-4 \overline{A \bar{C}}}}{2 \bar{A}}=\widetilde{F}, \\
& \bar{A}=r+c_{1} r \bar{H}, \\
& \bar{B}=-F_{\max }\left(r-\beta-\kappa \bar{H}+c_{1} \bar{H}(r-\beta)\right), \\
& \bar{C}=-F_{\max } \delta\left(1+c_{1} \bar{H}\right), \\
& \bar{H}=\frac{\eta}{q}=\widetilde{H},
\end{aligned}
$$

and

$$
\begin{aligned}
& \widehat{F}=\frac{-\widehat{B}+\sqrt{\widehat{B}^{2}-4 \widehat{A} \widehat{C}}}{2 \widehat{A}}, \\
& \widehat{A}=r+c_{1} r \widehat{H}, \\
& \widehat{B}=-F_{\max }\left(r-\beta-\kappa \widehat{H}+c_{1} \widehat{H}(r-\beta)\right),
\end{aligned}
$$




$$
\begin{aligned}
& \widehat{C}=-F_{\max } \delta\left(1+c_{1} \widehat{H}\right), \\
& \widehat{H}=\frac{\theta e^{-\mu_{3} \tau_{3}} \xi}{c g}, \quad \widetilde{S}=\frac{\xi}{g} .
\end{aligned}
$$

Numerical simulations for system (21)-(26) are performed using the following values: $\delta=$ $10, \beta=0.01, r=0.009, F_{\max }=1200, \varepsilon=0.3, \alpha=0.4, m=0.1, a=0.6, \lambda=0.1, \theta=1.2, c=4$, $\eta=0.1, \xi=0.03, d=0.8, h=0.1$, and $\mu_{i}=0.1(i=1,2,3)$. The other parameters will be chosen in what follows.

Let us consider the initial values:

IV1: $\psi_{\omega}^{1}=800, \psi_{\omega}^{2}=8, \psi_{\omega}^{3}=1, \psi_{\omega}^{4}=0.5, \psi_{\omega}^{5}=1, \psi_{\omega}^{6}=0.5$,

IV2: $\psi_{\omega}^{1}=600, \psi_{\omega}^{2}=10, \psi_{\omega}^{3}=3, \psi_{\omega}^{4}=1, \psi_{\omega}^{5}=3, \psi_{\omega}^{6}=1$, and

IV3: $\psi_{\omega}^{1}=400, \psi_{\omega}^{2}=12, \psi_{\omega}^{3}=5, \psi_{\omega}^{4}=2, \psi_{\omega}^{5}=5, \psi_{\omega}^{6}=2, \omega=-\bar{m},-\bar{m}+1, \ldots, 0$.

- Stability of equilibria

We choose $\tau_{1}=0.1, \tau_{2}=0.5, \tau_{3}=0.9$ and choose $\kappa, \eta$, and $\xi$ are varied as follows.

Case (1) $\kappa=0.003, q=0.05$, and $g=0.002$. This yields $\mathcal{R}_{0}=0.634<1$. Figure 1 shows that the concentration of healthy cells increases and tends to the value $F^{0}=1089.96$. Moreover, the concentrations of infected cells, free viruses, antibodies, and CTL cells decay and reach zero for IV1-IV3. Consequently, there exists only one equilibrium that is $Q^{0}$ and it is G.A.S. This result supports the result of Theorem 1.

Case (2) $\kappa=0.01, q=0.05$, and $g=0.002$. With these values we obtain $\mathcal{R}_{1}^{Y}=0.799<$ $1<\mathcal{R}_{0}=2.112$ and $\mathcal{R}_{1}^{Z}=0.440<1<\mathcal{R}_{0}=2.112$. Figure 2 displays that for all the three initial values IV1-IV3, the solutions of the system reach the equilibrium $Q^{*}=$ $(523.57,10.285,5.244,1.438,0,0)$. Consequently, $Q^{*}$ exists and it is G.A.S. This agrees with the result of Theorem 2 .

Case (3) $\kappa=0.01, q=0.2$, and $g=0.002$ and then $\mathcal{R}_{0}=2.112>1, \mathcal{R}_{1}^{Y}=1.587>1$, and $\mathcal{R}_{1}^{Z}=0.440<1$. Figure 3 displays that the solutions of the system reach the equilibrium $\bar{Q}=(823.137,5.676,2.894,0.5,2.934,0)$ for all the initial values IV1-IV3. Thus $\bar{Q}$ exists and it is G.A.S. This result is consistent with the result of Theorem 3.

Case (4) $\kappa=0.01, q=0.005$, and $g=0.01$ and then $\mathcal{R}_{0}=2.112>1, \mathcal{R}_{1}^{Z}=1.337>1$, and $\mathcal{R}_{1}^{Y} / \mathcal{R}_{2}^{Z}=0.041<1$. From Fig. 4 we can see that, for all the initial values IV1-IV3, the solutions of the system tend to the equilibrium $\widehat{Q}=(695.628,7.866,3,0.823,0,2.020)$. This result shows that $\widehat{Q}$ exists and it is G.A.S, and this agrees with the result of Theorem 4.

Case $(5) \kappa=0.01, q=0.3$, and $g=0.02$ and then $\mathcal{R}_{0}=2.112>1, \mathcal{R}_{1}^{Y}=1.742>1$, and $\mathcal{R}_{2}^{Z}=$ $1.412>1$. From Fig. 5 we observe that the solutions of the system reach the equilibrium $\widetilde{Q}=(901.96,4.153,1.5,0.333,1.169,2.469)$. This yields that $\widetilde{Q}$ exists and it is G.A.S. This illustrates the result of Theorem 5 .

- Impact of time delay on the viral dynamics

Without loss of generality we let $\tau=\tau_{1}=\tau_{2}=\tau_{3}$. We fix the values $\kappa=0.01, q=0.3$, and $g=0.02$ and select different values of $\tau$. We solve the system with initial IV2. Figure 6 shows the influence of the time delay parameter $\tau$ on the stability of the equilibria. One can see that as $\tau$ is increased, the concentration of healthy cells is increased, while the concentrations of infected cells, free viruses, CTL cells, and antibodies are decreased. Let us write $\mathcal{R}_{0}$ as follows:

$$
\mathcal{R}_{0}(\tau)=F^{0}\left(\frac{m(1-\varepsilon) e^{-\left(\mu_{1}+\mu_{3}\right) \tau}}{\alpha+m}+\varepsilon e^{-\left(\mu_{2}+\mu_{3}\right) \tau}\right)\left(\frac{\kappa \theta}{a c}\right) .
$$




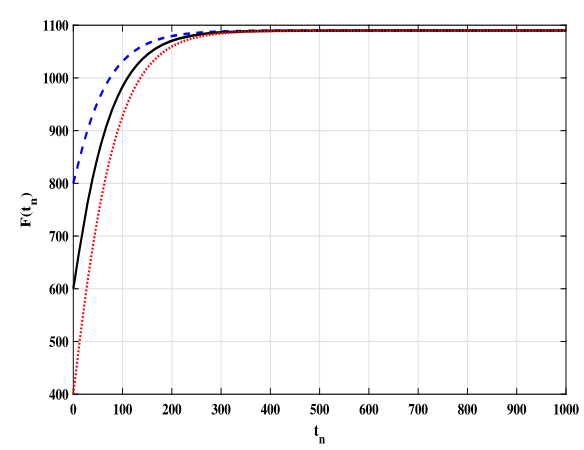

(a)

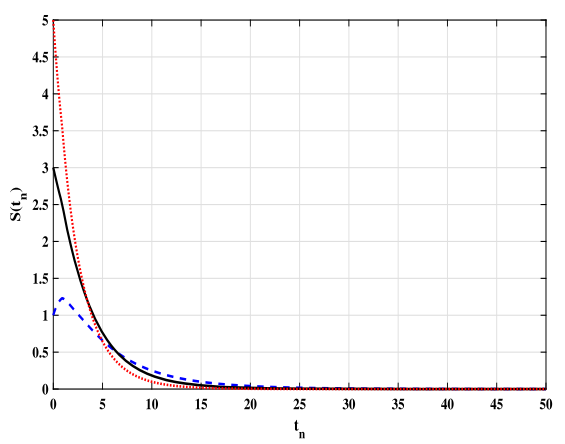

(c)

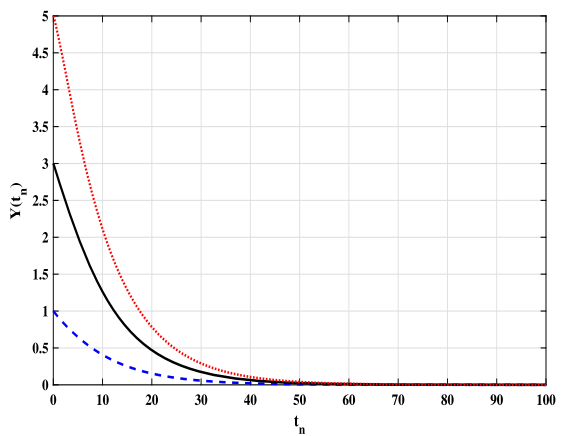

(e)

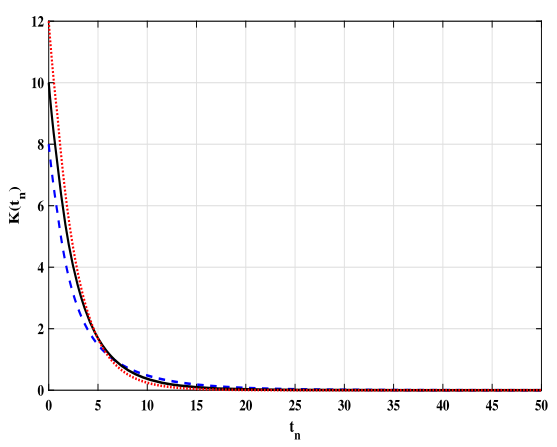

(b)

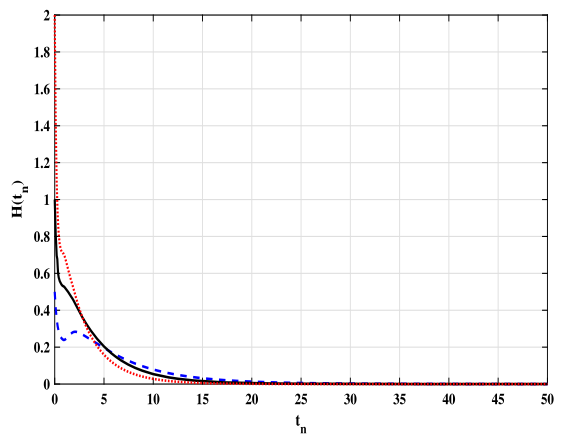

(d)

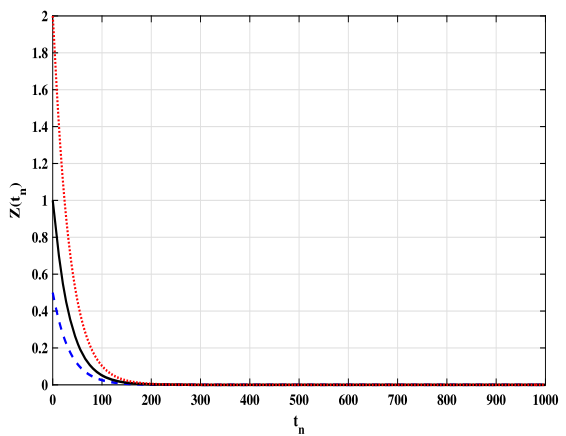

(f)

Figure 1 The simulation of trajectories of system (21)-(26) for Case $\mathcal{R}_{0} \leq 1$

Since $\mathcal{R}_{0}$ is a decreasing function of $\tau$, then the time delay can change the stability properties of equilibria. Using the values of the parameters and from Fig. 6 we can see that if $\tau \geq 4.373$, then $Q^{0}$ is G.A.S. Biologically, the time delays play a similar role of antiviral treatment in eliminating the viruses from the body.

\section{Conclusion}

In this paper, we formulated and analyzed a discrete-time viral infection model with both antibody and cell-mediated immune responses. We incorporated two categories of infected cells, namely latently infected cells (such cells contain the virus but are not producing) and actively infected cells (such cells produce new viruses). The production and removal rates of the cells and viruses as well as the incidence rate were modeled by general 


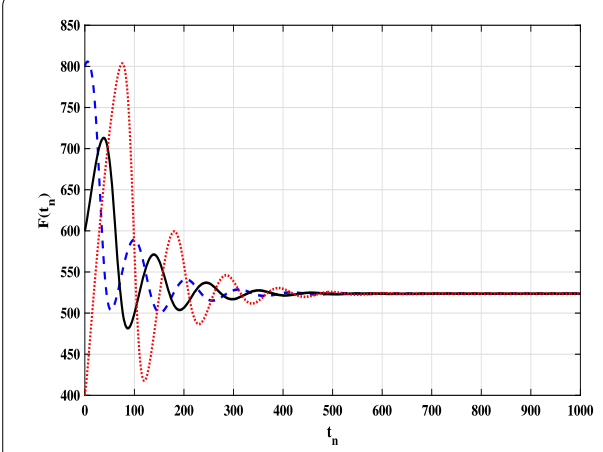

(a)

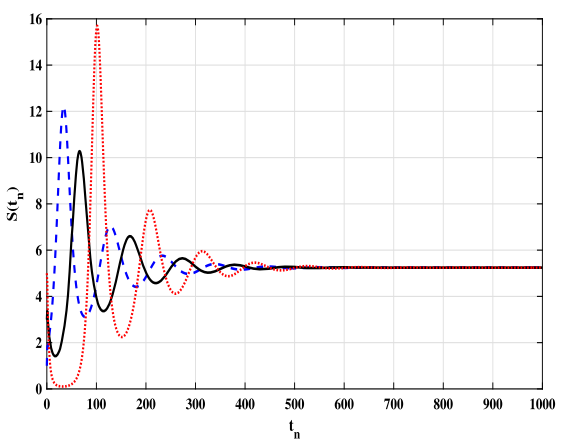

(c)

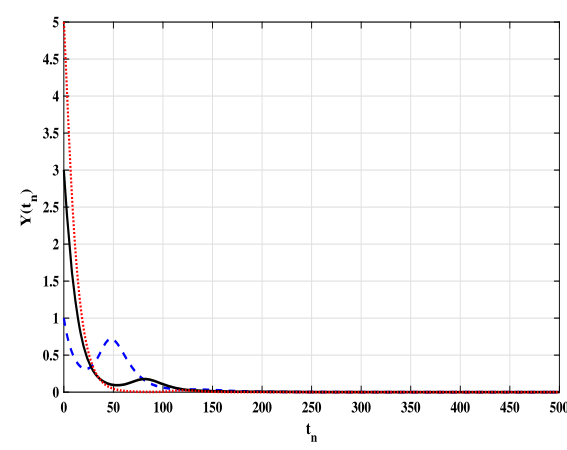

(e)

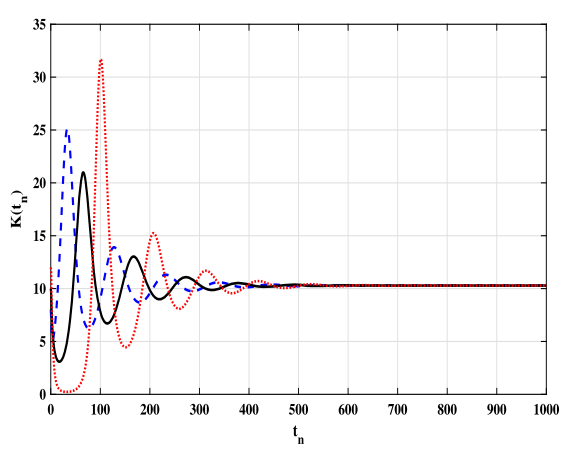

(b)

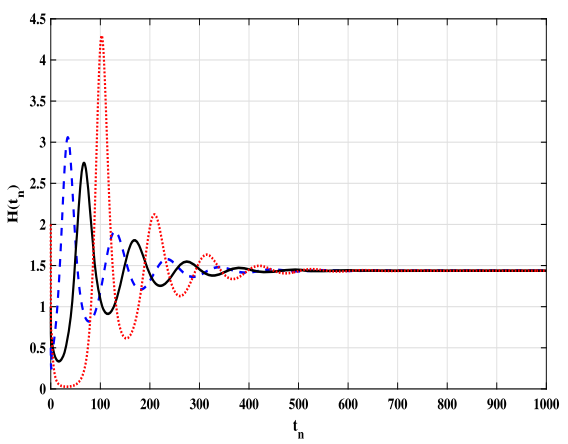

(d)

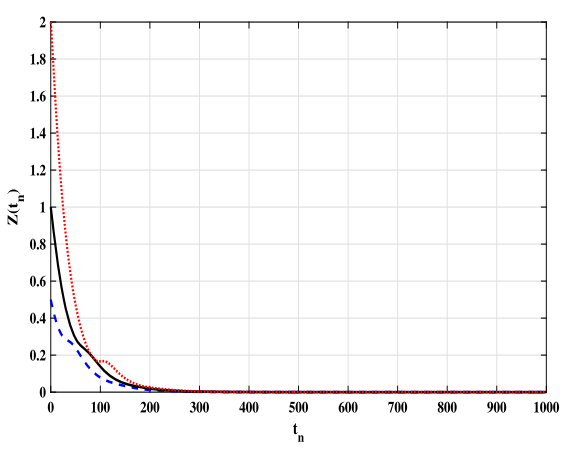

(f)

Figure 2 The simulation of trajectories of system (21)-(26) for Case $\mathcal{R}_{1}^{Y} \leq 1<\mathcal{R}_{0}$ and $\mathcal{R}_{1}^{Z} \leq 1<\mathcal{R}_{0}$

nonlinear functions which satisfy a set of conditions. These general functions encompass several specific forms commonly used in the virus dynamics literature. We incorporated three types of time delays, in which the first and second delays describe the times between a virus contacts a susceptible cell and the cell becomes latently infected and actively infected cell, respectively. The third delay is the time from death of an infected cell until the virus is active. We used nonstandard finite difference scheme to discretize the continuoustime model. We showed that the solutions of the discrete-time model with given initial states are positive and bounded. We derived four threshold parameters which fully determine the existence and stability of the five equilibria of the model. Then, we proved the global stability of the equilibria by constructing Lyapunov functions. Moreover, we performed numerical simulations to support the global stability results. We studied the 


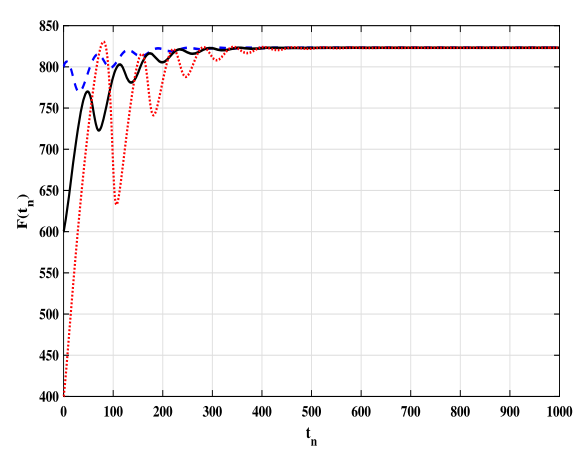

(a)

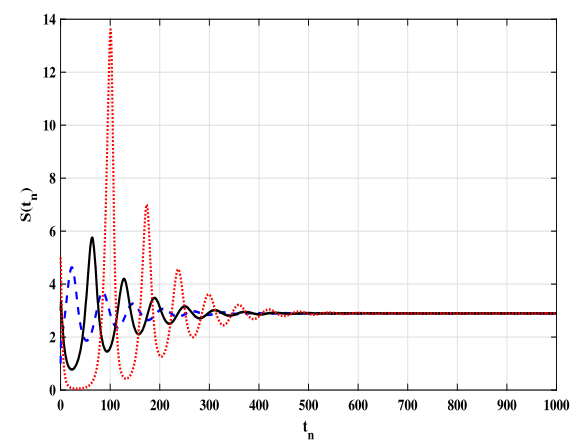

(c)

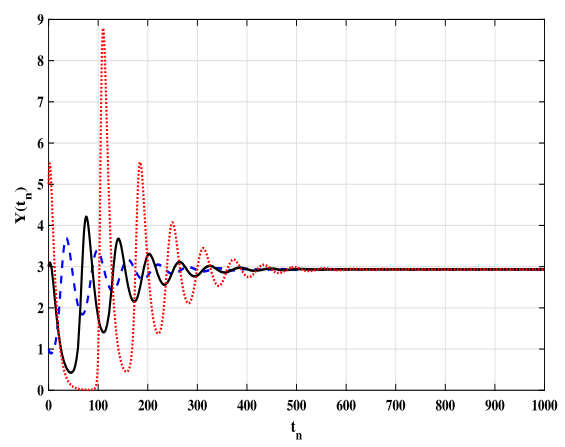

(e)

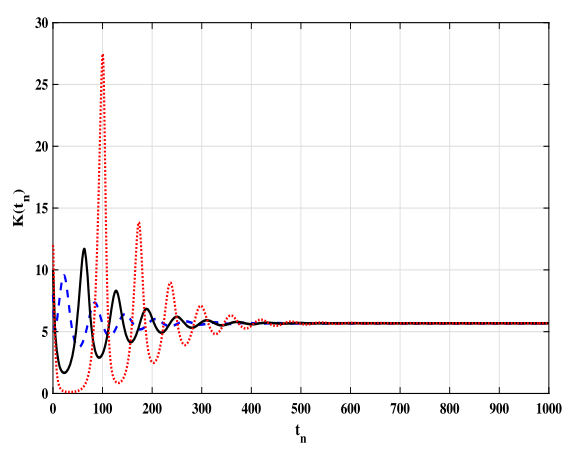

(b)

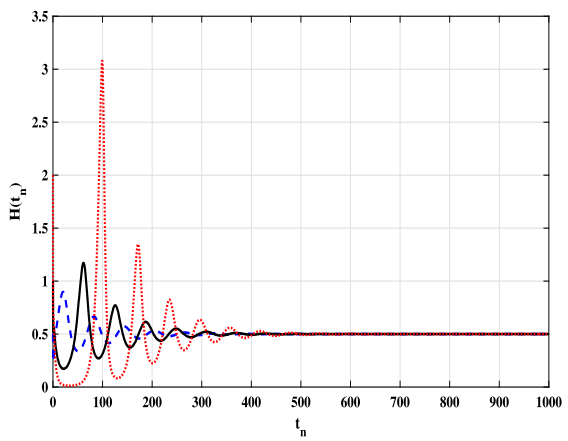

(d)

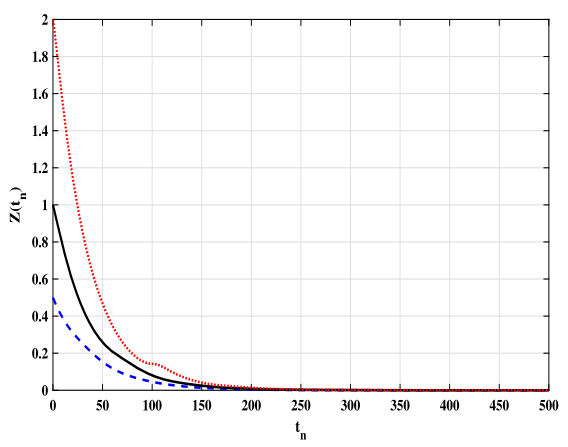

(f)

Figure 3 The simulation of trajectories of system (21)-(26) for Case $\mathcal{R}_{1}^{Y}>1$ and $\mathcal{R}_{1}^{Z} \leq 1$

effect of time delay on the virus dynamics. Since $Q^{0}$ is the desired equilibrium to be stabilized, we determined the critical time delay parameter $\tau^{\text {critical }}$ by solving the equation $\mathcal{R}_{0}\left(\tau^{\text {critical }}\right)=1$ and showed that $Q^{0}$ is globally asymptotically stable when $\tau \geq \tau^{\text {critical }}$. This shows that the time delay can have a similar effect as the antiviral drugs. This gives some impression to develop a new class of treatment to increase the delay period and then suppress the viral replication. It is worth emphasizing that the role of the delay term does not only take into account the delay in the dynamical response of the interacting entities, but also their heterogeneity. This can be accounted for by modeling interactions as shown in [63]. Recently, many authors have argued that the virus moves freely in body and follows the Fickian diffusion (see, e.g., [64-66]). Therefore, it is more reasonable to study reaction-diffusion versions of our model. We leave these points as possible future works. 


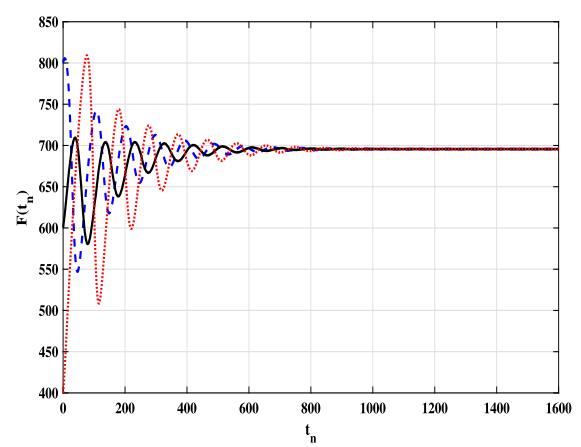

(a)

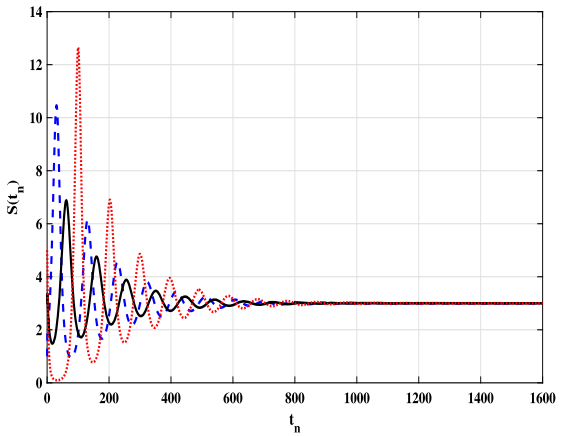

(c)

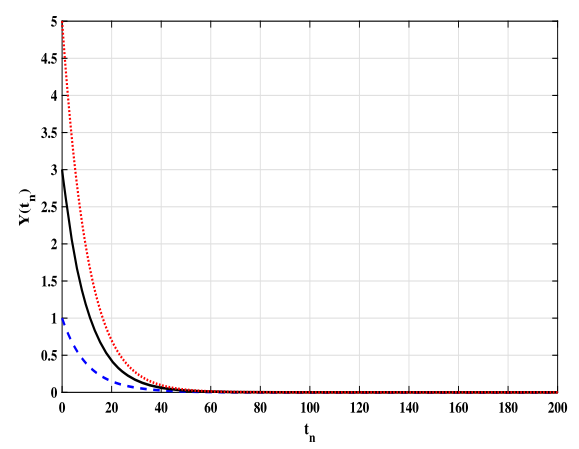

(e)

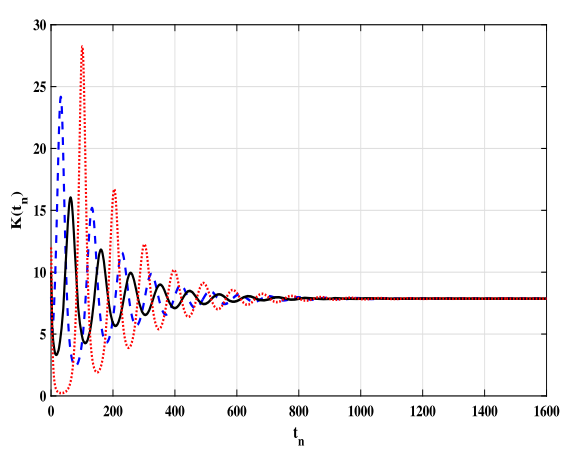

(b)

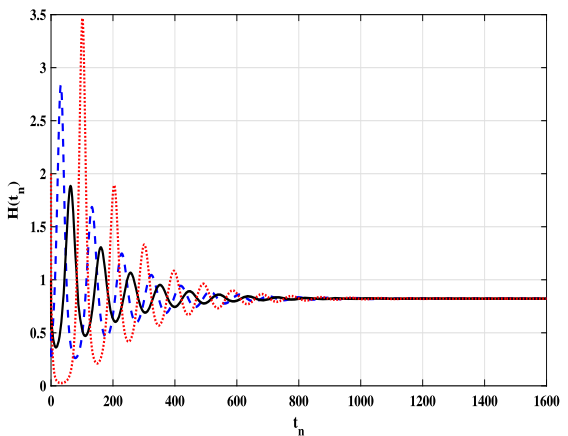

(d)

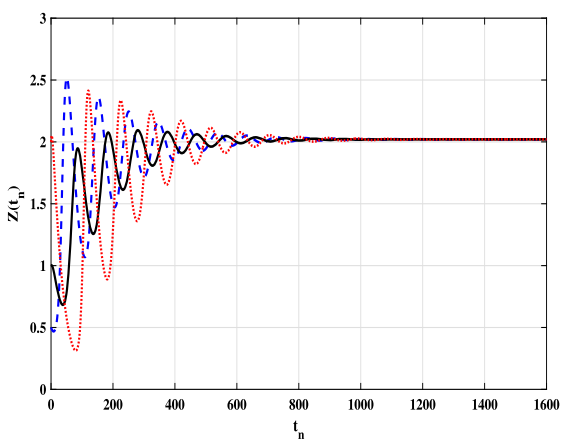

(f)

Figure 4 The simulation of trajectories of system (21)-(26) for Case $\mathcal{R}_{1}^{Z}>1$ and $\mathcal{R}_{1}^{Y} / \mathcal{R}_{2}^{Z} \leq 1$

\section{Appendix}

Proof of Lemma 1 First we put $n=0$ and show that there exists positive and unique $\left(F_{1}, K_{1}, S_{1}, H_{1}, Y_{1}, Z_{1}\right)$. From Eq. (11) we have

$$
F_{1}-F_{0}-\phi(h) \Theta\left(F_{1}\right)+\phi(h) \Lambda\left(F_{1}, H_{0}\right)=0 .
$$

We define a function $\chi_{1}(F)$ as follows:

$$
\chi_{1}(F)=F-F_{0}-\phi(h) \Theta(F)+\phi(h) \Lambda\left(F, H_{0}\right)=0 .
$$




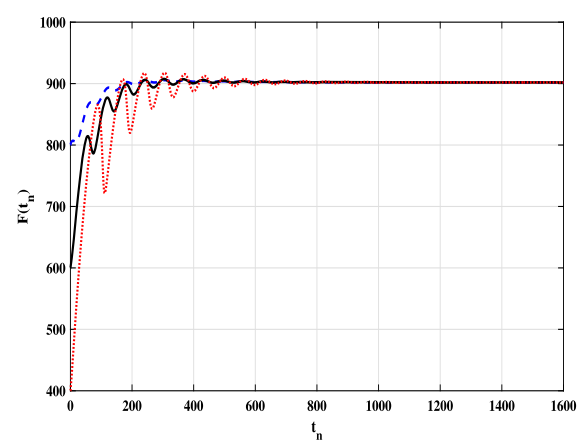

(a)

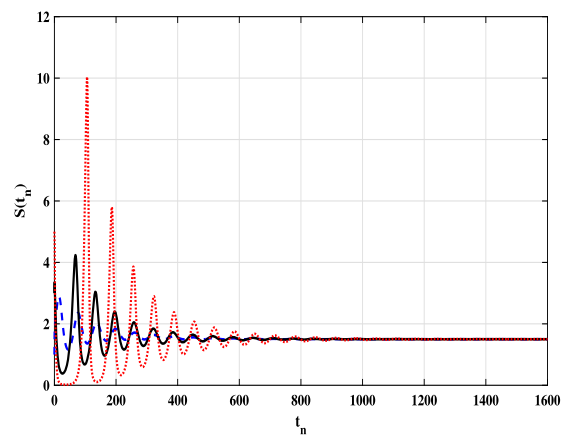

(c)

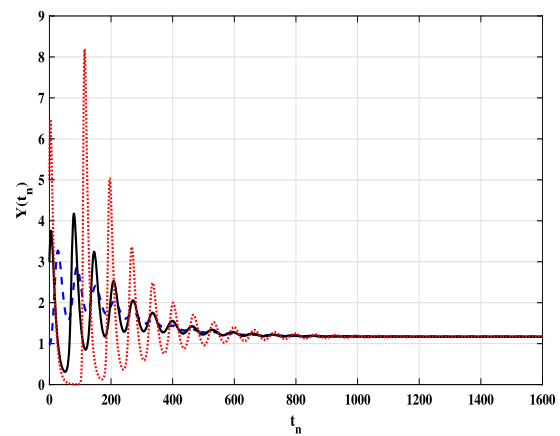

(e)

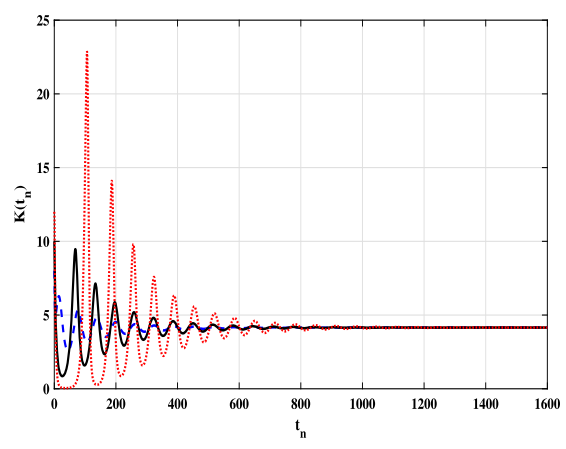

(b)

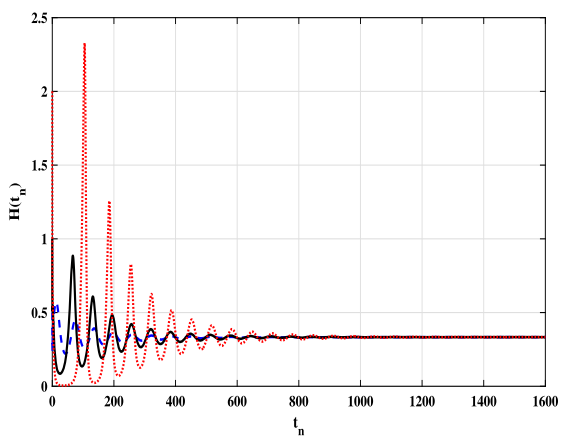

(d)

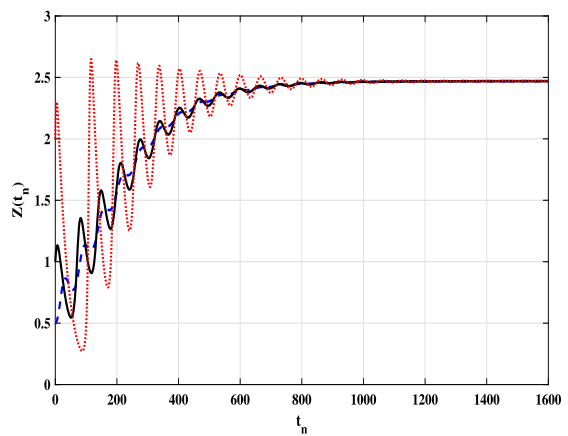

(f)

Figure 5 The simulation of trajectories of system (21)-(26) for Case $\mathcal{R}_{1}^{Y}>\mathcal{R}_{2}^{Z}>1$

According to Conditions $\mathrm{C} 1-\mathrm{C} 2$ we have $\chi_{1}$ is a strictly increasing function in $F$ and

$$
\begin{aligned}
& \chi_{1}(0)=-F_{0}-\phi(h) \Theta(0)<0, \\
& \lim _{F \rightarrow \infty} \chi_{1}(F)=\infty .
\end{aligned}
$$

Hence, there exists unique $F_{1}>0$ such that $\chi_{1}\left(F_{1}\right)=0$.

From Eq. (12) we have

$$
K_{1}-K_{0}-\phi(h)(1-\varepsilon) e^{-\mu_{1} \tau_{1}} \Lambda\left(F_{-m_{1}+1}, H_{-m_{1}}\right)+\phi(h)(\alpha+m) \digamma_{1}\left(K_{1}\right)=0 .
$$




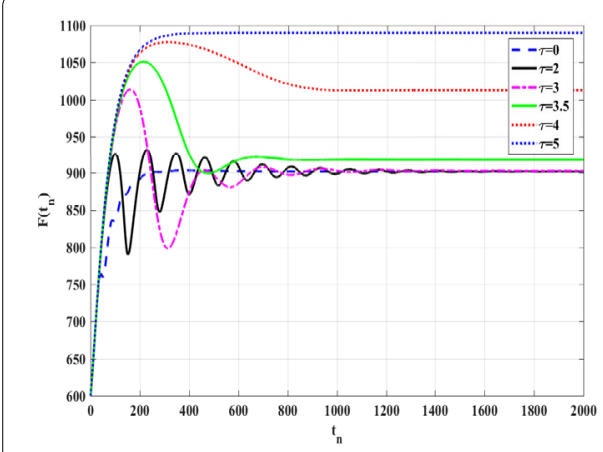

(a)

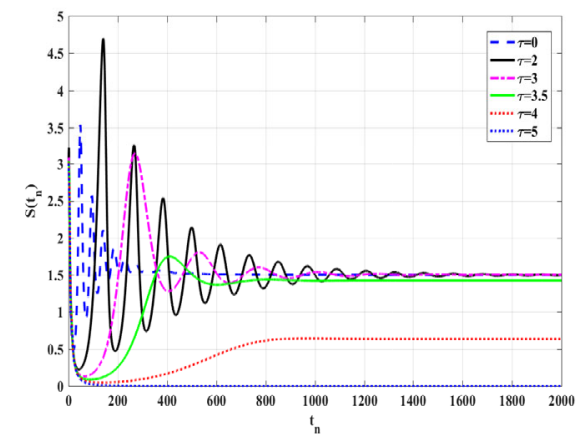

(c)

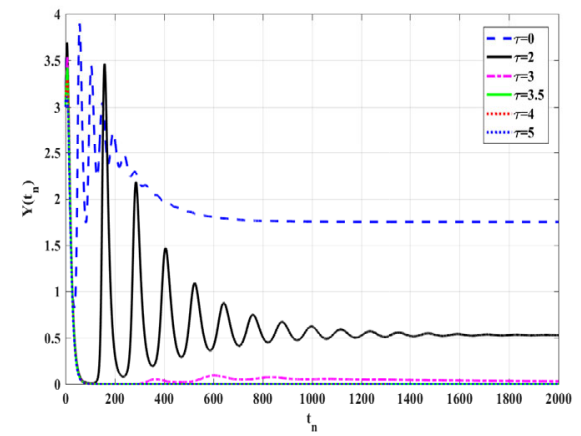

(e)

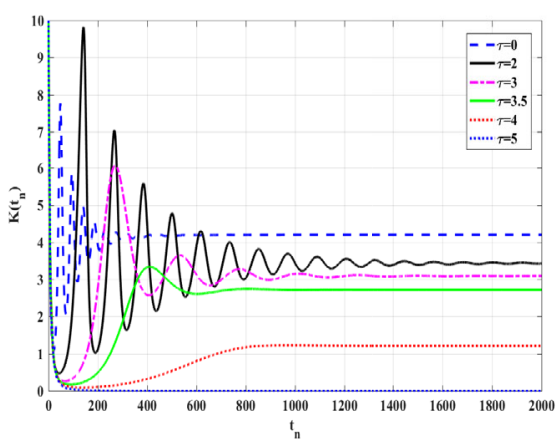

(b)

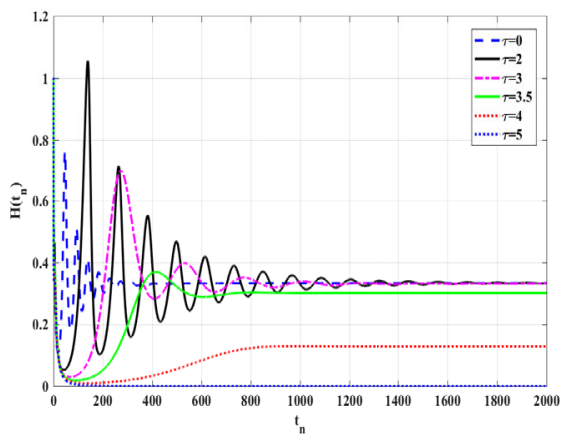

(d)

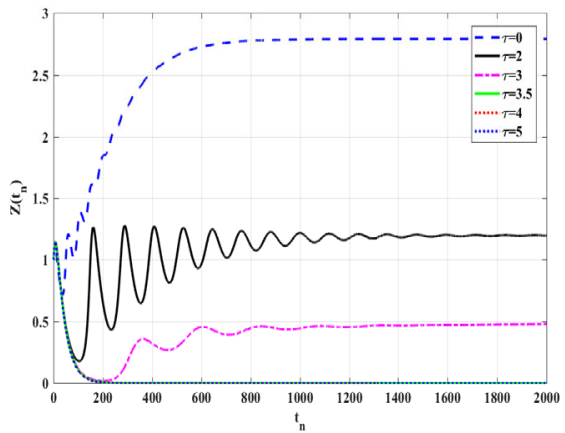

(f)

Figure 6 The simulation of trajectories of system (21)-(26) with different values of $\tau$

Define a function $\chi_{2}(K)$ as follows:

$$
\chi_{2}(K)=K-K_{0}-\phi(h)(1-\varepsilon) e^{-\mu_{1} \tau_{1}} \Lambda\left(F_{-m_{1}+1}, H_{-m_{1}}\right)+\phi(h)(\alpha+m) \digamma_{1}(K)=0 .
$$

Conditions $\mathrm{C} 2-\mathrm{C} 3$ imply that $\chi_{2}$ is a strictly increasing function in $K$. In addition,

$$
\begin{aligned}
& \chi_{2}(0)=-K_{0}-\phi(h)(1-\varepsilon) e^{-\mu_{1} \tau_{1}} \Lambda\left(F_{-m_{1}+1}, H_{-m_{1}}\right)<0, \\
& \lim _{K \rightarrow \infty} \chi_{2}(K)=\infty .
\end{aligned}
$$

It follows that there exists unique $K_{1} \in(0, \infty)$ such that $\chi_{2}\left(K_{1}\right)=0$. 
Since $\digamma_{2}\left(S_{1}\right)=S_{1}, \digamma_{3}\left(H_{1}\right)=H_{1}, \digamma_{4}\left(Y_{1}\right)=Y_{1}$, and $\digamma_{5}\left(Z_{1}\right)=Z_{1}$, then from Eqs. (13) and (16) we get

$$
\begin{aligned}
Z_{1}= & Z_{0}+\frac{\phi(h) g\left[S_{0}+\phi(h) \varepsilon e^{-\mu_{2} \tau_{2}} \Lambda\left(F_{-m_{2}+1}, H_{-m_{2}}\right)+\phi(h) m \digamma_{1}\left(K_{1}\right)\right]}{1+\phi(h)\left(a+\lambda Z_{1}\right)} Z_{1} \\
& -\phi(h) \xi Z_{1} .
\end{aligned}
$$

Then

$$
A_{1} Z_{1}^{2}+B_{1} Z_{1}+C_{1}=0
$$

where

$$
\begin{aligned}
A_{1}= & (1+\phi(h) \xi) \phi(h) \lambda, \\
B_{1}= & (1+\phi(h) \xi)(1+\phi(h) a)-\phi(h) \lambda Z_{0} \\
& -\phi(h) g\left[S_{0}+\phi(h) e^{-\mu_{2} \tau_{2}} \Lambda\left(F_{-m_{2}+1}, H_{-m_{2}}\right)+\phi(h) m \digamma_{1}\left(K_{1}\right)\right], \\
C_{1}= & -(1+\phi(h) a) Z_{0} .
\end{aligned}
$$

Since $A_{1}>0, C_{1}<0$, then $B_{1}^{2}-4 A_{1} C_{1}>0$, and hence there exists a unique positive root of Eq. (27) $Z_{1}>0$. It follows from Eq. (13)

$$
S_{1}=\frac{S_{0}+\phi(h) \varepsilon e^{-\mu_{2} \tau_{2}} \Lambda\left(F_{-m_{2}+1}, H_{-m_{2}}\right)+\phi(h) m \digamma_{1}\left(K_{1}\right)}{1+\phi(h)\left(a+\lambda Z_{1}\right)}>0 .
$$

Then we have $S_{1}>0$.

Now we show that $Y_{1}>0$. From Eqs. (14)-(15) we get

$$
Y_{1}=Y_{0}+\frac{\phi(h) q\left(H_{0}+\phi(h) \theta e^{-\mu_{3} \tau_{3}} S_{-m_{3}+1}\right)}{1+\phi(h)\left(c+d Y_{1}\right)} Y_{1}-\phi(h) \eta Y_{1} .
$$

Then we get

$$
A_{2} Y_{1}^{2}+B_{2} Y_{1}+C_{2}=0
$$

where

$$
\begin{aligned}
& A_{2}=(1+\phi(h) \eta) \phi(h) d \\
& B_{2}=(1+\phi(h) \eta)(1+\phi(h) c)-\phi(h) d Y_{0}-\phi(h) q\left[H_{0}+\phi(h) \theta e^{-\mu_{3} \tau_{3}} \digamma_{2}\left(S_{-m_{3}+1}\right)\right] \\
& C_{2}=-(1+\phi(h) c) Y_{0} .
\end{aligned}
$$

Since $A_{2}>0, C_{2}<0$, then $B_{2}^{2}-4 A_{2} C_{2}>0$, and hence there exists a unique positive root of Eq. (28) $Y_{1}>0$.

From Eq. (14) we get

$$
H_{1}=\frac{H_{0}+\phi(h) \theta e^{-\mu_{3} \tau_{3}} S_{-m_{3}+1}}{1+\phi(h)\left(c+d Y_{1}\right)}>0
$$

Therefore, the solution $\left(F_{1}, K_{1}, S_{1}, H_{1}, Y_{1}, Z_{1}\right)$ exists uniquely and is positive. 
Repeating the above process for $n=1$, we can prove that $\left(F_{2}, K_{2}, S_{2}, H_{2}, Y_{2}, Z_{2}\right)$ exists uniquely and is positive. Therefore, mathematical induction yields that, for all $n \in \mathbb{N}$, $\left(F_{n}, K_{n}, S_{n}, H_{n}, Y_{n}, Z_{n}\right)$ exists uniquely and is positive.

By induction, we obtain $F_{n}>0, K_{n}>0, S_{n}>0, H_{n}>0, Y_{n}>0$, and $Z_{n}>0 \forall n \geq 0$. To investigate the boundedness of solution, from Eq. (11) we have

$$
\frac{F_{n+1}-F_{n}}{\phi(h)} \leq \Theta\left(F_{n+1}\right) \leq b-\bar{b} F_{n+1}
$$

Hence

$$
F_{n+1} \leq \frac{F_{n}}{1+\phi(h) \bar{b}}+\frac{\phi(h) b}{1+\phi(h) \bar{b}}
$$

By Lemma 2.2 in [67] we have

$$
F_{n} \leq\left(\frac{1}{1+\phi(h) \bar{b}}\right)^{n} F_{0}+\frac{b}{\bar{b}}\left[1-\left(\frac{1}{1+\phi(h) \bar{b}}\right)^{n}\right]
$$

which implies that $\lim _{n \rightarrow \infty} \sup F_{n} \leq b / \bar{b} \leq \vartheta_{1}$. Define

$$
\Omega_{n}=(1-\varepsilon) e^{-\mu_{1} \tau_{1}} F_{n-m_{1}}+\varepsilon e^{-\mu_{2} \tau_{2}} F_{n-m_{2}}+K_{n}+S_{n}+\frac{\lambda}{g} Z_{n} .
$$

Then

$$
\begin{aligned}
\Omega_{n+1}-\Omega_{n}= & (1-\varepsilon) e^{-\mu_{1} \tau_{1}}\left(F_{n-m_{1}+1}-F_{n-m_{1}}\right)+\varepsilon e^{-\mu_{2} \tau_{2}}\left(F_{n-m_{2}+1}-F_{n-m_{2}}\right) \\
& +K_{n+1}-K_{n}+S_{n+1}-S_{n}+\frac{\lambda}{g}\left(Z_{n+1}-Z_{n}\right) \\
= & (1-\varepsilon) e^{-\mu_{1} \tau_{1}} \phi(h)\left[\Theta\left(F_{n-m_{1}+1}\right)-\Lambda\left(F_{n-m_{1}+1}, H_{n-m_{1}}\right)\right] \\
& +\varepsilon e^{-\mu_{2} \tau_{2}} \phi(h)\left[\Theta\left(F_{n-m_{2}+1}\right)-\Lambda\left(F_{n-m_{2}+1}, H_{n-m_{2}}\right)\right] \\
& +\phi(h)\left[(1-\varepsilon) e^{-\mu_{1} \tau_{1}} \Lambda\left(F_{n-m_{1}+1}, H_{n-m_{1}}\right)-\left(\alpha+m_{1}\right) \digamma_{1}\left(K_{n+1}\right)\right] \\
& +\phi(h)\left[\varepsilon e^{-\mu_{2} \tau_{2}} \Lambda\left(F_{n-m_{2}+1}, H_{n-m_{2}}\right)+m_{1}\left(K_{n+1}\right)-a \digamma_{2}\left(S_{n+1}\right)\right. \\
& \left.-\lambda \digamma_{2}\left(S_{n+1}\right) \digamma_{5}\left(Z_{n+1}\right)\right] \\
& +\phi(h) \frac{\lambda}{g}\left[g \digamma_{2}\left(S_{n+1}\right) \digamma_{5}\left(Z_{n+1}\right)-\xi \digamma_{5}\left(Z_{n+1}\right)\right] \\
= & \phi(h)\left[(1-\varepsilon) e^{-\mu_{1} \tau_{1}} \Theta\left(F_{n-m_{1}+1}\right)+\varepsilon e^{-\mu_{2} \tau_{2}} \Theta\left(F_{n-m_{2}+1}\right)-\alpha \digamma_{1}\left(K_{n+1}\right)\right. \\
& \left.-a S_{n+1}-\frac{\lambda \xi}{g} Z_{n+1}\right] .
\end{aligned}
$$

According to Conditions $\mathrm{C} 1$ and $\mathrm{C} 3$, we have

$$
\begin{aligned}
\Omega_{n+1}-\Omega_{n} \leq & \phi(h)\left[(1-\varepsilon) e^{-\mu_{1} \tau_{1}}\left(b-\bar{b} F_{n-m_{1}+1}\right)+\varepsilon e^{-\mu_{2} \tau_{2}}\left(b-\bar{b} F_{n-m_{2}+1}\right)-\alpha v_{1} K_{n+1}\right. \\
& \left.-a S_{n+1}-\frac{\lambda \xi}{g} Z_{n+1}\right] .
\end{aligned}
$$


We have

$$
(1-\varepsilon) e^{-\mu_{1} \tau_{1}} b+\varepsilon e^{-\mu_{2} \tau_{2}} b \leq b(1-\varepsilon)+b \varepsilon=b
$$

Then

$$
\begin{aligned}
& \Omega_{n+1}-\Omega_{n} \\
& \quad \leq \phi(h) b-\phi(h) \sigma_{1}\left[(1-\varepsilon) e^{-\mu_{1} \tau_{1}} F_{n-m_{1}+1}+\varepsilon e^{-\mu_{2} \tau_{2}} F_{n-m_{2}+1}+K_{n+1}+S_{n+1}+\frac{\lambda}{g} Z_{n+1}\right] \\
& \quad=\phi(h) b-\phi(h) \sigma_{1} \Omega_{n+1} .
\end{aligned}
$$

Hence

$$
\Omega_{n+1} \leq \frac{\Omega_{n}}{1+\phi(h) \sigma_{1}}+\frac{\phi(h) b}{1+\phi(h) \sigma_{1}} .
$$

Consequently, we get $\lim _{n \rightarrow \infty} \sup \Omega_{n} \leq \vartheta_{1}, \lim _{n \rightarrow \infty} \sup K_{n} \leq \vartheta_{1}, \lim _{n \rightarrow \infty} \sup S_{n} \leq \vartheta_{1}$, and $\lim _{n \rightarrow \infty} \sup Z_{n} \leq \vartheta_{2}$. Thus, for any $\varpi>0$, there exists an integer $\varrho_{\varpi}>0$ such that $S_{n} \leq$ $\vartheta_{1}+\varpi$ for $n \geq \varrho_{\varpi}$. We define

$$
\Psi_{n}=H_{n}+\frac{d}{q} Y_{n}
$$

Then

$$
\begin{aligned}
\Psi_{n+1}-\Psi_{n} & =H_{n+1}-H_{n}+\frac{d}{q}\left(Y_{n+1}-Y_{n}\right) \\
& =\phi(h)\left(\theta e^{-\mu_{3} \tau_{3}} S_{n-m_{3}+1}-c H_{n+1}-\frac{d \eta}{q} Y_{n+1}\right) \\
& \leq \phi(h)\left(\theta e^{-\mu_{3} \tau_{3}}\left(\vartheta_{1}+\varpi\right)-c H_{n+1}-\frac{d \eta}{q} Y_{n+1}\right) \\
& \leq \phi(h)\left(\theta e^{-\mu_{3} \tau_{3}}\left(\vartheta_{1}+\varpi\right)-\sigma_{2} \Psi_{n+1}\right) \quad \text { for } n \geq \varrho_{\varpi}+m_{3} .
\end{aligned}
$$

Then $\lim _{n \rightarrow \infty} \sup \Psi_{n} \leq \frac{\theta e^{-\mu_{3} \tau_{3}\left(\vartheta_{1}+\varpi\right)}}{\sigma_{2}} \leq \frac{\theta\left(\vartheta_{1}+\varpi\right)}{\sigma_{2}}$. The arbitrariness of $\varpi$ yields that $\lim _{n \rightarrow \infty} \sup \Psi_{n} \leq \frac{\theta \vartheta_{1}}{\sigma_{2}}=\vartheta_{3}$. Hence, $\lim _{n \rightarrow \infty} \sup H_{n} \leq \vartheta_{3}$ and $\lim _{n \rightarrow \infty} \sup Y_{n} \leq \vartheta_{4}$. Therefore, the solution $\left(F_{n}, K_{n}, S_{n}, H_{n}, Y_{n}, Z_{n}\right)$ converges to $\Gamma$ as $n \rightarrow \infty$.

Proof of Lemma 2 The equilibria of system (11)-(16) satisfy:

$$
\begin{aligned}
& \Theta(F)-\Lambda(F, H)=0, \\
& (1-\varepsilon) e^{-\mu_{1} \tau_{1}} \Lambda(\digamma, H)-(\alpha+m) \digamma_{1}(K)=0, \\
& \varepsilon e^{-\mu_{2} \tau_{2}} \Lambda(\digamma, H)+m \digamma_{1}(K)-a \digamma_{2}(S)-\lambda \digamma_{2}(S) \digamma_{5}(Z)=0, \\
& \theta e^{-\mu_{3} \tau_{3}} \digamma_{2}(S)-c \digamma_{3}(H)-d \digamma_{3}(H) \digamma_{4}(Y)=0, \\
& q \digamma_{3}(H) \digamma_{4}(Y)-\eta \digamma_{4}(Y)=0, \\
& g \lambda \digamma_{2}(S) \digamma_{5}(Z)-\xi \digamma_{5}(Z)=0 .
\end{aligned}
$$


From Eq. (33), either $\digamma_{4}(Y)=0$ or $\digamma_{4}(Y) \neq 0\left(\digamma_{3}(H)=\frac{\eta}{q}\right)$. By solving Eq. (34), we get $\digamma_{5}(Z)=0$ or $\digamma_{5}(Z) \neq 0\left(\digamma_{2}(S)=\frac{\xi}{g}\right)$. If Condition C3 $\digamma_{4}(Y)=0$ and $\digamma_{5}(Z)=0$ imply that $Y=0$ and $Z=0$, thus we have the following possibilities:

1. $Y=Z=0$, then Condition C3 implies that $\digamma_{i}^{-1}, i=1, \ldots, 5$, exist and they are strictly increasing functions. From Eqs. (29)-(32) we get

$$
\begin{aligned}
& K=\digamma_{1}^{-1}\left(\frac{(1-\varepsilon) e^{-\mu_{1} \tau_{1}} \Theta(F)}{\alpha+m}\right)=\pi_{1}(F), \\
& S=\digamma_{2}^{-1}\left(\frac{\gamma e^{\mu_{3} \tau_{3}} \Theta(F)}{a}\right)=\pi_{2}(F), \\
& H=\digamma_{3}^{-1}\left(\frac{\theta \gamma \Theta(F)}{a c}\right)=\pi_{3}(F),
\end{aligned}
$$

where

$$
\gamma=\frac{m(1-\varepsilon) e^{-\mu_{1} \tau_{1}-\mu_{3} \tau_{3}}}{\alpha+m}+\varepsilon e^{-\mu_{2} \tau_{2}-\mu_{3} \tau_{3}} .
$$

Obviously, $\pi_{i}(0)>0$ and $\pi_{i}\left(F^{0}\right)=0, i=1,2$, 3. Substituting Eq. (37) into Eq. (32), we obtain

$$
\frac{\theta \gamma}{a} \Lambda\left(F, \pi_{3}(F)\right)-c \digamma_{3}\left(\pi_{3}(F)\right)=0
$$

Equation (39) admits a solution $F=F^{0}$, which gives $K=S=H=0$ and leads to the pathogen-free equilibrium $Q^{0}=\left(F^{0}, 0,0,0,0,0\right)$. Let

$$
\psi_{1}(F)=\frac{\theta \gamma}{a} \Lambda\left(F, \pi_{3}(F)\right)-c \digamma_{3}\left(\pi_{3}(F)\right)=0
$$

Then from Conditions C1-C3 we have

$$
\begin{aligned}
& \psi_{1}(0)=-c \digamma_{3}\left(\pi_{3}(0)\right)<0, \\
& \psi_{1}\left(F^{0}\right)=0 .
\end{aligned}
$$

Moreover,

$$
\begin{aligned}
& \psi_{1}^{\prime}(F)=\frac{\theta \gamma}{a}\left[\frac{\partial \Lambda}{\partial F}+\pi_{3}^{\prime}(F) \frac{\partial \Lambda}{\partial H}\right]-c \pi_{3}^{\prime}(F) \digamma_{3}^{\prime}\left(\pi_{3}(F)\right) \\
& \psi_{1}^{\prime}\left(F^{0}\right)=\frac{\theta \gamma}{a}\left[\frac{\partial \Lambda\left(F^{0}, 0\right)}{\partial F}+\pi_{3}^{\prime}\left(F^{0}\right) \frac{\partial \Lambda\left(F^{0}, 0\right)}{\partial H}\right]-c \pi_{3}^{\prime}\left(F^{0}\right) \digamma_{3}^{\prime}(0) .
\end{aligned}
$$

Condition $\mathrm{C} 2$ implies that $\frac{\partial \Lambda\left(F^{0}, 0\right)}{\partial F}=0$. Also, from Condition C3, we have $\digamma_{3}^{\prime}(0)>0$, then

$$
\begin{aligned}
\psi_{1}^{\prime}\left(F^{0}\right) & =c \pi_{3}^{\prime}\left(F^{0}\right) \digamma_{3}^{\prime}(0)\left(\frac{\theta \gamma}{a c \digamma_{3}^{\prime}(0)} \frac{\partial \Lambda\left(F^{0}, 0\right)}{\partial H}-1\right) \\
& =\frac{\theta \gamma \Theta^{\prime}\left(F^{0}\right)}{a}\left(\frac{\theta \gamma}{a c \digamma_{3}^{\prime}(0)} \frac{\partial \Lambda\left(F^{0}, 0\right)}{\partial H}-1\right) .
\end{aligned}
$$


From Condition $\mathrm{C} 1$, we have $\Theta^{\prime}\left(F^{0}\right)<0$. Therefore, if

$$
\frac{\theta \gamma}{a c \digamma^{\prime}(0)} \frac{\partial \Lambda\left(F^{0}, 0\right)}{\partial H}>1
$$

hence $\psi_{1}^{\prime}\left(F^{0}\right)<0$ and there exists $F^{*} \in\left(0, F^{0}\right)$ such that $\psi_{1}\left(F^{*}\right)=0$. From Eqs. (35)-(37) we obtain $K^{*}=\pi_{1}\left(F^{*}\right)>0, S^{*}=\pi_{2}\left(F^{*}\right)>0$, and $H^{*}=\pi_{3}\left(F^{*}\right)>0$. Therefore, a persistent infection equilibrium without immune response $Q^{*}\left(F^{*}, K^{*}, H^{*}, S^{*}, 0,0\right)$ exists when $\frac{\theta \gamma}{a c \digamma_{3}^{\prime}(0)} \frac{\partial \Lambda\left(F^{0}, 0\right)}{\partial H}>1$. Let us define

$$
\mathcal{R}_{0}=\frac{\theta \gamma}{a c \digamma_{3}^{\prime}(0)} \frac{\partial \Lambda\left(F^{0}, 0\right)}{\partial H}
$$

2. $Y \neq 0$ and $Z=0$, we have $\bar{H}=\digamma_{3}^{-1}\left(\frac{\eta}{q}\right)>0$. Let $H=\bar{H}$ in Eq. (29) and define $\psi_{2}$ as follows:

$$
\psi_{2}(F)=\Theta(F)-\Lambda(F, \bar{H})=0 .
$$

According to Conditions $\mathrm{C} 1$ and $\mathrm{C} 2$, we have

$$
\psi_{2}(0)=\Theta(0)>0 \quad \text { and } \quad \psi_{2}\left(F^{0}\right)=-\Lambda\left(F^{0}, \bar{H}\right)<0 .
$$

Since $\psi_{2}(F)$ is a strictly decreasing function of $F$, then there exists unique $\bar{F} \in\left(0, F^{0}\right)$ such that $\psi_{2}(\bar{F})=0$. Now from Eqs. (32), (35), and (36) we obtain

$$
\bar{K}=\pi_{1}(\bar{F}), \quad \bar{S}=\pi_{2}(\bar{F}), \quad \bar{Y}=\digamma_{4}^{-1}\left(\frac{c}{d}\left(\frac{\theta \gamma \Lambda(\bar{F}, \bar{H})}{a c \digamma_{3}(\bar{H})}-1\right)\right) .
$$

Clearly, $\bar{K}>0$ and $\bar{S}>0$; moreover, $\bar{Y}>0$ when $\frac{\theta \gamma \Lambda(\bar{F}, \bar{H})}{a c \digamma_{3}(\bar{H})}>1$. Now we define

$$
\mathcal{R}_{1}^{Y}=\frac{\theta \gamma \Lambda(\bar{F}, \bar{H})}{\operatorname{ac\digamma }{ }_{3}(\bar{H})} .
$$

Hence, $\bar{Y}$ can be rewritten as $\bar{Y}=\digamma_{4}^{-1}\left(\frac{c}{d}\left(\mathcal{R}_{1}^{Y}-1\right)\right)$. It follows that there exists a persistent infection equilibrium with only humoral immune response $\bar{Q}(\bar{F}, \bar{K}, \bar{S}, \bar{H}, \bar{Y}, 0)$ if $\mathcal{R}_{1}^{Y}>1$.

3. $Z \neq 0$ and $Y=0$, we have $\widehat{S}=\digamma_{2}^{-1}\left(\frac{\xi}{g}\right)>0$. Let $S=\widehat{S}$ in Eq. (32), then we have

$$
\widehat{H}=\digamma_{3}^{-1}\left(\frac{\theta e^{-\mu_{3} \tau_{3}} \xi}{c g}\right)>0 .
$$

Let $H=\widehat{H}$ in Eq. (29) and define $\psi_{3}$ as follows:

$$
\psi_{3}(F)=\Theta(F)-\Lambda(F, \widehat{H})=0 .
$$

Clearly,

$$
\psi_{3}(0)=\Theta(0)>0 \quad \text { and } \quad \psi_{3}\left(F^{0}\right)=-\Lambda\left(F^{0}, \widehat{H}\right)<0
$$


According to Conditions $\mathrm{C} 1$ and $\mathrm{C} 2$, there exists unique $\widehat{F} \in\left(0, F^{0}\right)$ such that $\psi_{3}(\widehat{F})=0$. From Eq. (35) we conclude that $\widehat{K}=\pi_{1}(\widehat{F})>0$. Now from Eqs. (30)-(32) we have $\widehat{Z}=$ $\digamma_{5}^{-1}\left(\frac{a}{\lambda}\left(\mathcal{R}_{1}^{Z}-1\right)\right)$, where

$$
\mathcal{R}_{1}^{Z}=\frac{\theta \gamma \Lambda(\widehat{F}, \widehat{H})}{a c \digamma_{3}(\widehat{H})} .
$$

Consequently, there exists a persistent infection equilibrium with only CTL immune response $\widehat{Q}(\widehat{F}, \widehat{K}, \widehat{S}, \widehat{H}, 0, \widehat{Z})$ if $\mathcal{R}_{1}^{Z}>1$.

4. $Z \neq 0$ and $Y \neq 0$, we have $\widetilde{H}=\bar{H}=\digamma_{3}^{-1}\left(\frac{\eta}{q}\right)>0$ and $\widetilde{S}=\widehat{S}=\digamma_{2}^{-1}\left(\frac{\xi}{g}\right)>0$. Let $H=\widetilde{H}$ in Eq. (29) and define $\psi_{4}$ as follows:

$$
\psi_{4}(F)=\Theta(F)-\Lambda(F, \tilde{H})=0 .
$$

Clearly,

$$
\psi_{4}(0)=\Theta(0)>0 \quad \text { and } \quad \psi_{4}\left(F^{0}\right)=-\Lambda\left(F^{0}, \tilde{H}\right)<0 .
$$

According to $\mathrm{C} 1$ and $\mathrm{C} 2$, there exists unique $\widetilde{F} \in\left(0, F^{0}\right)$ such that $\psi_{4}(\widetilde{F})=0$. Thus, we conclude from Eq. (35) that $\widetilde{K}=\pi_{1}(\widetilde{F})>0$. Now from Eqs. (30)-(32) we have

$$
\widetilde{Z}=\digamma_{5}^{-1}\left(\frac{a}{\lambda}\left(\mathcal{R}_{2}^{Z}-1\right)\right) \text { and } \tilde{Y}=\digamma_{4}^{-1}\left(\frac{c}{d}\left(\left(\mathcal{R}_{1}^{Y} / \mathcal{R}_{2}^{Z}\right)-1\right)\right)>0 \text {, }
$$

where

$$
\mathcal{R}_{2}^{Z}=\frac{e^{\mu_{3} \tau_{3}} \gamma \Lambda(\widetilde{F}, \widetilde{H})}{a \digamma_{2}(\widetilde{S})} \quad \text { and } \quad \mathcal{R}_{1}^{Y} / \mathcal{R}_{2}^{Z}=\frac{\theta \digamma_{2}(\widetilde{S}) \Lambda(\bar{F}, \bar{H})}{c \digamma_{3}(\widetilde{H}) \Lambda(\widetilde{F}, \widetilde{H})}=\frac{\theta e^{-\mu_{3} \tau_{3}} \xi q}{c g \eta}
$$

It follows that there exists a persistent infection equilibrium with both humoral and CTL immune responses $\widetilde{Q}(\widetilde{F}, \widetilde{K}, \widetilde{S}, \widetilde{H}, \widetilde{Y}, \widetilde{Z})$ if $\mathcal{R}_{1}^{Y}>\mathcal{R}_{2}^{Z}>1$.

Proof of Theorem 1 Define

$$
\begin{aligned}
\mathcal{L}_{n}= & \frac{1}{\phi(h)}\left[\gamma\left(F_{n}-F^{0}-\int_{F^{0}}^{F_{n}} \lim _{H \rightarrow 0^{+}} \frac{\Lambda\left(F^{0}, H\right)}{\Lambda(\zeta, H)} d \varsigma\right)+\frac{m e^{-\mu_{3} \tau_{3}}}{\alpha+m} K_{n}+e^{-\mu_{3} \tau_{3}} S_{n}+\frac{a}{\theta} H_{n}\right. \\
& \left.+\frac{a d}{\theta q} Y_{n}+\frac{\lambda e^{-\mu_{3} \tau_{3}}}{g} Z_{n}\right]+\frac{a c}{\theta} \digamma_{3}\left(H_{n}\right)+\frac{a d \eta}{\theta q} \digamma_{4}\left(Y_{n}\right)+\frac{\lambda \xi e^{-\mu_{3} \tau_{3}}}{g} \digamma_{5}\left(Z_{n}\right) \\
& +\frac{m(1-\varepsilon) e^{-\mu_{1} \tau_{1}-\mu_{3} \tau_{3}}}{\alpha+m} \sum_{j=n-m_{1}}^{n-1} \Lambda\left(F_{j+1}, H_{j}\right)+\varepsilon e^{-\mu_{2} \tau_{2}-\mu_{3} \tau_{3}} \sum_{j=n-m_{2}}^{n-1} \Lambda\left(F_{j+1}, H_{j}\right) \\
& +a e^{-\mu_{3} \tau_{3}} \sum_{j=n-m_{3}}^{n-1} \digamma_{2}\left(S_{j+1}\right),
\end{aligned}
$$

where $\gamma$ is defined by Eq. (38). Hence, $\mathcal{L}_{n}>0$ for all $F_{n}, K_{n}, S_{n}, H_{n}, Y_{n}, Z_{n}>0$, and $\mathcal{L}_{n}=0$ if and only if $F_{n}=F^{0}, K_{n}=0, S_{n}=0, H_{n}=0, Y_{n}=0$, and $Z_{n}=0$. We compute the difference 
$\Delta \mathcal{L}_{n}=\mathcal{L}_{n+1}-\mathcal{L}_{n}$ as follows:

$$
\begin{aligned}
& \Delta \mathcal{L}_{n}=\frac{1}{\phi(h)}\left[\gamma\left(F_{n+1}-F^{0}-\int_{F^{0}}^{F_{n+1}} \lim _{H \rightarrow 0^{+}} \frac{\Lambda\left(F^{0}, H\right)}{\Lambda(\varsigma, H)} d \varsigma\right)\right. \\
& +\frac{m e^{-\mu_{3} \tau_{3}}}{\alpha+m} K_{n+1}+e^{-\mu_{3} \tau_{3}} S_{n+1}+\frac{a}{\theta} H_{n+1} \\
& \left.+\frac{a d}{\theta q} Y_{n+1}+\frac{\lambda e^{-\mu_{3} \tau_{3}}}{g} Z_{n+1}\right]+\frac{a c}{\theta} \digamma_{3}\left(H_{n+1}\right)+\frac{a d \eta}{\theta q} \digamma_{4}\left(Y_{n+1}\right) \\
& +\frac{\lambda \xi e^{-\mu_{3} \tau_{3}}}{g} \digamma_{5}\left(Z_{n+1}\right) \\
& +\frac{m(1-\varepsilon) e^{-\mu_{1} \tau_{1}-\mu_{3} \tau_{3}}}{\alpha+m} \sum_{j=n-m_{1}+1}^{n} \Lambda\left(F_{j+1}, H_{j}\right) \\
& +\varepsilon e^{-\mu_{2} \tau_{2}-\mu_{3} \tau_{3}} \sum_{j=n-m_{2}+1}^{n} \Lambda\left(F_{j+1}, H_{j}\right)+a e^{-\mu_{3} \tau_{3}} \sum_{j=n-m_{3}+1}^{n} \digamma_{2}\left(S_{j+1}\right) \\
& -\frac{1}{\phi(h)}\left[\gamma\left(F_{n}-F^{0}-\int_{F^{0}}^{F_{n}} \lim _{H \rightarrow 0^{+}} \frac{\Lambda\left(F^{0}, H\right)}{\Lambda(\varsigma, H)} d \varsigma\right)+\frac{m e^{-\mu_{3} \tau_{3}}}{\alpha+m} K_{n}\right. \\
& +e^{-\mu_{3} \tau_{3}} S_{n}+\frac{a}{\theta} H_{n}+\frac{a d}{\theta q} Y_{n} \\
& \left.+\frac{\lambda e^{-\mu_{3} \tau_{3}}}{g} Z_{n}\right]-\frac{a c}{\theta} \digamma_{3}\left(H_{n}\right)-\frac{a d \eta}{\theta q} \digamma_{4}\left(Y_{n}\right)-\frac{\lambda \xi e^{-\mu_{3} \tau_{3}}}{g} \digamma_{5}\left(Z_{n}\right) \\
& -\frac{m(1-\varepsilon) e^{-\mu_{1} \tau_{1}-\mu_{3} \tau_{3}}}{\alpha+m} \sum_{j=n-m_{1}}^{n-1} \Lambda\left(F_{j+1}, H_{j}\right) \\
& -\varepsilon e^{-\mu_{2} \tau_{2}-\mu_{3} \tau_{3}} \sum_{j=n-m_{2}}^{n-1} \Lambda\left(F_{j+1}, H_{j}\right)-a e^{-\mu_{3} \tau_{3}} \sum_{j=n-m_{3}}^{n-1} \digamma_{2}\left(S_{j+1}\right) \\
& \Delta \mathcal{L}_{n}=\frac{1}{\phi(h)}\left[\gamma\left(F_{n+1}-F_{n}-\int_{F_{n}}^{F_{n+1}} \lim _{H \rightarrow 0^{+}} \frac{\Lambda\left(F^{0}, H\right)}{\Lambda(\varsigma, H)} d \varsigma\right)+\frac{m e^{-\mu_{3} \tau_{3}}}{\alpha+m}\left(K_{n+1}-K_{n}\right)\right. \\
& +e^{-\mu_{3} \tau_{3}}\left(S_{n+1}-S_{n}\right)+\frac{a}{\theta}\left(H_{n+1}-H_{n}\right)+\frac{a d}{\theta q}\left(Y_{n+1}-Y_{n}\right) \\
& \left.+\frac{\lambda e^{-\mu_{3} \tau_{3}}}{g}\left(Z_{n+1}-Z_{n}\right)\right] \\
& +\frac{a c}{\theta}\left[\digamma_{3}\left(H_{n+1}\right)-\digamma_{3}\left(H_{n}\right)\right]+\frac{a d \eta}{\theta q}\left[\digamma_{4}\left(Y_{n+1}\right)-\digamma_{4}\left(Y_{n}\right)\right] \\
& +\frac{\lambda \xi e^{-\mu_{3} \tau_{3}}}{g}\left[\digamma_{5}\left(Z_{n+1}\right)-\digamma_{5}\left(Z_{n}\right)\right] \\
& +\frac{m(1-\varepsilon) e^{-\mu_{1} \tau_{1}-\mu_{3} \tau_{3}}}{\alpha+m}\left(\sum_{j=n-m_{1}+1}^{n} \Lambda\left(F_{j+1}, H_{j}\right)-\sum_{j=n-m_{1}}^{n-1} \Lambda\left(F_{j+1}, H_{j}\right)\right) \\
& +\varepsilon e^{-\mu_{2} \tau_{2}-\mu_{3} \tau_{3}}\left(\sum_{j=n-m_{2}+1}^{n} \Lambda\left(F_{j+1}, H_{j}\right)-\sum_{j=n-m_{2}}^{n-1} \Lambda\left(F_{j+1}, H_{j}\right)\right) \\
& +a e^{-\mu_{3} \tau_{3}}\left(\sum_{j=n-m_{3}+1}^{n} \digamma_{2}\left(S_{j+1}\right)-\sum_{j=n-m_{3}}^{n-1} \digamma_{2}\left(S_{j+1}\right)\right) .
\end{aligned}
$$


Using Lemma 3.1 [68], we get

$$
\begin{aligned}
\lim _{H \rightarrow 0^{+}} \frac{\Lambda\left(F^{0}, H\right)}{\Lambda\left(F_{n+1}, H\right)}\left(F_{n+1}-F_{n}\right) & \leq \int_{F_{n}}^{F_{n+1}} \lim _{H \rightarrow 0^{+}} \frac{\Lambda\left(F^{0}, H\right)}{\Lambda(\varsigma, H)} d \varsigma \\
& \leq \lim _{H \rightarrow 0^{+}} \frac{\Lambda\left(F^{0}, H\right)}{\Lambda\left(F_{n}, H\right)}\left(F_{n+1}-F_{n}\right) .
\end{aligned}
$$

Hence

$$
\begin{aligned}
\Delta \mathcal{L}_{n} \leq & \frac{1}{\phi(h)}\left[\gamma\left(1-\lim _{H \rightarrow 0^{+}} \frac{\Lambda\left(F^{0}, H\right)}{\Lambda\left(F_{n+1}, H\right)}\right)\left(F_{n+1}-F_{n}\right)\right. \\
& +\frac{m e^{-\mu_{3} \tau_{3}}}{\alpha+m}\left(K_{n+1}-K_{n}\right)+e^{-\mu_{3} \tau_{3}}\left(S_{n+1}-S_{n}\right) \\
& \left.+\frac{a}{\theta}\left(H_{n+1}-H_{n}\right)+\frac{a d}{\theta q}\left(Y_{n+1}-Y_{n}\right)+\frac{\lambda e^{-\mu_{3} \tau_{3}}}{g}\left(Z_{n+1}-Z_{n}\right)\right] \\
& +\frac{a c}{\theta}\left[\digamma_{3}\left(H_{n+1}\right)-\digamma_{3}\left(H_{n}\right)\right] \\
& +\frac{a d \eta}{\theta q}\left[\digamma_{4}\left(Y_{n+1}\right)-\digamma_{4}\left(Y_{n}\right)\right]+\frac{\lambda \xi e^{-\mu_{3} \tau_{3}}}{g}\left[\digamma_{5}\left(Z_{n+1}\right)-\digamma_{5}\left(Z_{n}\right)\right] \\
& +\frac{m(1-\varepsilon) e^{-\mu_{1} \tau_{1}-\mu_{3} \tau_{3}}}{\alpha+m}\left[\Lambda\left(F_{n+1}, H_{n}\right)-\Lambda\left(F_{n-m_{1}+1}, H_{n-m_{1}}\right)\right] \\
& +\varepsilon e^{-\mu_{2} \tau_{2}-\mu_{3} \tau_{3}}\left[\Lambda\left(F_{n+1}, H_{n}\right)-\Lambda\left(F_{n-m_{2}+1}, H_{n-m_{2}}\right)\right] \\
& +a e^{-\mu_{3} \tau_{3}}\left[\digamma_{2}\left(S_{n+1}\right)-\digamma_{2}\left(S_{n-m_{3}+1}\right)\right] .
\end{aligned}
$$

From Eqs. (11)-(16) we have

$$
\begin{aligned}
\Delta \mathcal{L}_{n} \leq & \gamma\left(1-\lim _{H \rightarrow 0^{+}} \frac{\Lambda\left(F^{0}, H\right)}{\Lambda\left(F_{n+1}, H\right)}\right)\left(\Theta\left(F_{n+1}\right)-\Lambda\left(F_{n+1}, H_{n}\right)\right) \\
& +\frac{m e^{-\mu_{3} \tau_{3}}}{\alpha+m}\left[(1-\varepsilon) e^{-\mu_{1} \tau_{1}} \Lambda\left(F_{n-m_{1}+1}, H_{n-m_{1}}\right)-(\alpha+m) \digamma_{1}\left(K_{n+1}\right)\right] \\
& +e^{-\mu_{3} \tau_{3}}\left[\varepsilon e^{-\mu_{2} \tau_{2}} \Lambda\left(F_{n-m_{2}+1}, H_{n-m_{2}}\right)+m \digamma_{1}\left(K_{n+1}\right)-a \digamma_{2}\left(S_{n+1}\right)\right. \\
& \left.-\lambda \digamma_{2}\left(S_{n+1}\right) \digamma_{5}\left(Z_{n+1}\right)\right] \\
& +\frac{a}{\theta}\left[\theta e^{-\mu_{3} \tau_{3}} \digamma_{2}\left(S_{n-m_{3}+1}\right)-c \digamma_{3}\left(H_{n+1}\right)-d \digamma_{3}\left(H_{n+1}\right) \digamma_{4}\left(Y_{n+1}\right)\right] \\
& +\frac{a d}{\theta q}\left[q \digamma_{3}\left(H_{n+1}\right) \digamma_{4}\left(Y_{n+1}\right)-\eta \digamma_{4}\left(Y_{n+1}\right)\right] \\
& +\frac{\lambda e^{-\mu_{3} \tau_{3}}}{g}\left[g \digamma_{2}\left(S_{n+1}\right) \digamma_{5}\left(Z_{n+1}\right)-\xi \digamma_{5}\left(Z_{n+1}\right)\right] \\
& +\frac{a c}{\theta}\left[\digamma_{3}\left(H_{n+1}\right)-\digamma_{3}\left(H_{n}\right)\right]+\frac{a d \eta}{\theta q}\left[\digamma_{4}\left(Y_{n+1}\right)-\digamma_{4}\left(Y_{n}\right)\right] \\
& +\frac{\lambda \xi e^{-\mu_{3} \tau_{3}}}{g}\left[\digamma_{5}\left(Z_{n+1}\right)-\digamma_{5}\left(Z_{n}\right)\right] \\
& +\frac{m(1-\varepsilon) e^{-\mu_{1} \tau_{1}-\mu_{3} \tau_{3}}}{\alpha+m}\left[\Lambda\left(F_{n+1}, H_{n}\right)-\Lambda\left(F_{n-m_{1}+1}, H_{n-m_{1}}\right)\right] \\
& +\varepsilon e^{-\mu_{2} \tau_{2}-\mu_{3} \tau_{3}}\left[\Lambda\left(F_{n+1}, H_{n}\right)-\Lambda\left(F_{n-m_{2}+1}, H_{n-m_{2}}\right)\right]
\end{aligned}
$$




$$
\begin{aligned}
& +a e^{-\mu_{3} \tau_{3}}\left[\digamma_{2}\left(S_{n+1}\right)-\digamma_{2}\left(S_{n-m_{3}+1}\right)\right] \\
= & \gamma\left(1-\lim _{H \rightarrow 0^{+}} \frac{\Lambda\left(F^{0}, H\right)}{\Lambda\left(F_{n+1}, H\right)}\right) \Theta\left(F_{n+1}\right)+\gamma \lim _{H \rightarrow 0^{+}} \frac{\Lambda\left(F^{0}, H\right)}{\Lambda\left(F_{n+1}, H\right)} \Lambda\left(F_{n+1}, H_{n}\right) \\
& -\frac{a c}{\theta} \digamma_{3}\left(H_{n}\right)-\frac{a d \eta}{\theta q} \digamma_{4}\left(Y_{n}\right)-\frac{\lambda \xi e^{-\mu_{3} \tau_{3}}}{g} \digamma_{5}\left(Z_{n}\right) .
\end{aligned}
$$

Using $\Theta\left(F^{0}\right)=0$, we obtain

$$
\begin{aligned}
\Delta \mathcal{L}_{n} \leq & \gamma\left(1-\frac{\partial \Lambda\left(F^{0}, 0\right) / \partial H}{\partial \Lambda\left(F_{n+1}, 0\right) / \partial H}\right)\left(\Theta\left(F_{n+1}\right)-\Theta\left(F^{0}\right)\right)+\gamma \frac{\partial \Lambda\left(F^{0}, 0\right) / \partial H}{\partial \Lambda\left(F_{n+1}, 0\right) / \partial H} \Lambda\left(F_{n+1}, H_{n}\right) \\
& -\frac{a c}{\theta} \digamma_{3}\left(H_{n}\right)-\frac{a d \eta}{\theta q} \digamma_{4}\left(Y_{n}\right)-\frac{\lambda \xi e^{-\mu_{3} \tau_{3}}}{g} \digamma_{5}\left(Z_{n}\right) \\
= & \gamma\left(1-\frac{\partial \Lambda\left(F^{0}, 0\right) / \partial H}{\partial \Lambda\left(F_{n+1}, 0\right) / \partial H}\right)\left(\Theta\left(F_{n+1}\right)-\Theta\left(F^{0}\right)\right) \\
& +\frac{a c}{\theta}\left(\frac{\gamma \theta}{a c} \frac{\partial \Lambda\left(F^{0}, 0\right) / \partial H}{\partial \Lambda\left(F_{n+1}, 0\right) / \partial H} \frac{\Lambda\left(F_{n+1}, H_{n}\right)}{\digamma_{3}\left(H_{n}\right)}-1\right) \digamma_{3}\left(H_{n}\right) \\
& -\frac{a d \eta}{\theta q} \digamma_{4}\left(Y_{n}\right)-\frac{\lambda \xi e^{-\mu_{3} \tau_{3}}}{g} \digamma_{5}\left(Z_{n}\right) .
\end{aligned}
$$

From Condition C4 we have

$$
\frac{\Lambda\left(F_{n+1}, H_{n}\right)}{\digamma_{3}\left(H_{n}\right)} \leq \lim _{H \rightarrow 0^{+}} \frac{\Lambda\left(F_{n+1}, H\right)}{\digamma_{3}(H)}=\frac{\partial \Lambda\left(F_{n+1}, 0\right) / \partial H}{\digamma_{3}^{\prime}(0)} .
$$

Then we get

$$
\begin{aligned}
\Delta \mathcal{L}_{n} \leq & \gamma\left(1-\frac{\partial \Lambda\left(F^{0}, 0\right) / \partial H}{\partial \Lambda\left(F_{n+1}, 0\right) / \partial H}\right)\left(\Theta\left(F_{n+1}\right)-\Theta\left(F^{0}\right)\right) \\
& +\frac{a c}{\theta}\left(\frac{\gamma \theta}{a c} \frac{\partial \Lambda\left(F^{0}, 0\right) / \partial H}{\digamma_{3}^{\prime}(0)}-1\right) \digamma_{3}\left(H_{n}\right) \\
& -\frac{a d \eta}{\theta q} \digamma_{4}\left(Y_{n}\right)-\frac{\lambda \xi e^{-\mu_{3} \tau_{3}}}{g} \digamma_{5}\left(Z_{n}\right) \\
= & \gamma\left(1-\frac{\partial \Lambda\left(F^{0}, 0\right) / \partial H}{\partial \Lambda\left(F_{n+1}, 0\right) / \partial H}\right)\left(\Theta\left(F_{n+1}\right)-\Theta\left(F^{0}\right)\right)+\frac{a c}{\theta}\left(\mathcal{R}_{0}-1\right) \digamma_{3}\left(H_{n}\right) \\
& -\frac{a d \eta}{\theta q} \digamma_{4}\left(Y_{n}\right)-\frac{\lambda \xi e^{-\mu_{3} \tau_{3}}}{g} \digamma_{5}\left(Z_{n}\right) .
\end{aligned}
$$

Conditions $\mathrm{C} 1$ and $\mathrm{C} 2$ imply that

$$
\left(1-\frac{\partial \Lambda\left(F^{0}, 0\right) / \partial H}{\partial \Lambda\left(F_{n+1}, 0\right) / \partial H}\right)\left(\Theta\left(F_{n+1}\right)-\Theta\left(F^{0}\right)\right) \leq 0 .
$$

Hence, if $\mathcal{R}_{0} \leq 1$, then we have $\Delta \mathcal{L}_{n} \leq 0$ for all $n \geq 0$. Obviously, $\lim _{n \rightarrow \infty} \Delta \mathcal{L}_{n}=0$ if $\lim _{n \rightarrow \infty} F_{n}=F^{0}, \lim _{n \rightarrow \infty}\left(\mathcal{R}_{0}-1\right) \digamma_{3}\left(H_{n}\right)=0, \lim _{n \rightarrow \infty} \digamma_{4}\left(Y_{n}\right)=0$, and $\lim _{n \rightarrow \infty} \digamma_{5}\left(Z_{n}\right)=0$. We have two cases:

- If $\mathcal{R}_{0}<1$, then $\lim _{n \rightarrow \infty} \digamma_{3}\left(H_{n}\right)=0, \lim _{n \rightarrow \infty} \digamma_{4}\left(Y_{n}\right)=0, \lim _{n \rightarrow \infty} \digamma_{5}\left(Z_{n}\right)=0$, and from Condition C3 we get $\lim _{n \rightarrow \infty} H_{n}=0, \lim _{n \rightarrow \infty} Y_{n}=0$, and $\lim _{n \rightarrow \infty} Z_{n}=0$, then we get from Eqs. (13)-(14) $\lim _{n \rightarrow \infty} K_{n}=0$ and $\lim _{n \rightarrow \infty} S_{n}=0$. 
- If $\mathcal{R}_{0}=1$, then $\lim _{n \rightarrow \infty} \Delta K_{n}=0$ when $\lim _{n \rightarrow \infty} Y_{n}=0, \lim _{n \rightarrow \infty} Z_{n}=0$, and $\lim _{n \rightarrow \infty} F_{n}=F^{0}$, and from Eq. (11) we obtain $\lim _{n \rightarrow \infty} \Lambda\left(F^{0}, H_{n}\right)=0$, then $\lim _{n \rightarrow \infty} H_{n}=0$. Moreover, from Eqs. (13)-(14) we get $\lim _{n \rightarrow \infty} K_{n}=0, \lim _{n \rightarrow \infty} S_{n}=0$. Hence $Q^{0}$ is G.A.S.

Proof of Lemma 3 From Conditions $\mathrm{C} 1$ and $\mathrm{C} 2$, for $F^{*}, \bar{F}, \widehat{F}, S^{*}, \widehat{S}, H^{*}, \bar{H}, \widehat{H}>0$, we have

$$
\begin{aligned}
& \left(F^{*}-\bar{F}\right)\left(\Theta(\bar{F})-\Theta\left(F^{*}\right)\right)>0, \\
& \left(\bar{F}-F^{*}\right)\left(\Lambda(\bar{F}, \bar{H})-\Lambda\left(F^{*}, \bar{H}\right)\right)>0, \\
& \left(\bar{H}-H^{*}\right)\left(\Lambda\left(F^{*}, \bar{H}\right)-\Lambda\left(F^{*}, H^{*}\right)\right)>0, \\
& \left(\bar{H}-H^{*}\right)\left(\Lambda(\bar{F}, \bar{H})-\Lambda\left(\bar{F}, H^{*}\right)\right)>0 .
\end{aligned}
$$

Using Condition C4, we get

$$
\left(H^{*}-\bar{H}\right)\left(\frac{\Lambda\left(F^{*}, \bar{H}\right)}{\digamma_{3}(\bar{H})}-\frac{\Lambda\left(F^{*}, H^{*}\right)}{\digamma_{3}\left(H^{*}\right)}\right)>0 .
$$

Suppose that $\operatorname{sgn}\left(\bar{F}-F^{*}\right)=\operatorname{sgn}\left(\bar{H}-H^{*}\right)$. For the equilibria $Q^{*}$ and $\bar{Q}$, we have

$$
\begin{aligned}
\Theta(\bar{F})-\Theta\left(F^{*}\right) & =\Lambda(\bar{F}, \bar{H})-\Lambda\left(F^{*}, H^{*}\right) \\
& =\left(\Lambda(\bar{F}, \bar{H})-\Lambda\left(F^{*}, \bar{H}\right)\right)+\left(\Lambda\left(F^{*}, \bar{H}\right)-\Lambda\left(F^{*}, H^{*}\right)\right) .
\end{aligned}
$$

Therefore, from inequalities (40)-(43) we get

$$
\operatorname{sgn}\left(F^{*}-\bar{F}\right)=\operatorname{sgn}\left(\bar{F}-F^{*}\right),
$$

which leads to a contradiction. Thus, $\operatorname{sgn}\left(\bar{F}-F^{*}\right)=\operatorname{sgn}\left(H^{*}-\bar{H}\right)$. Using the equilibrium conditions for $Q^{*}$, we have $\frac{\theta \gamma \Lambda\left(F^{*}, H^{*}\right)}{a c \digamma_{3}\left(H^{*}\right)}=1$, then

$$
\begin{aligned}
\mathcal{R}_{1}^{Y-1} & =\frac{\theta \gamma \Lambda(\bar{F}, \bar{H})}{a c \digamma_{3}(\bar{H})}-\frac{\theta \gamma \Lambda\left(F^{*}, H^{*}\right)}{a c \digamma_{3}\left(H^{*}\right)} \\
& =\frac{\theta \gamma}{a c}\left[\frac{\Lambda(\bar{F}, \bar{H})}{\digamma_{3}(\bar{H})}-\frac{\Lambda\left(F^{*}, H^{*}\right)}{\digamma_{3}\left(H^{*}\right)}\right] \\
& =\frac{\theta \gamma}{a c}\left[\frac{1}{\digamma_{3}(\bar{H})}\left(\Lambda(\bar{F}, \bar{H})-\Lambda\left(F^{*}, \bar{H}\right)\right)+\frac{\Lambda\left(F^{*}, \bar{H}\right)}{\digamma_{3}(\bar{H})}-\frac{\Lambda\left(F^{*}, H^{*}\right)}{\digamma_{3}\left(H^{*}\right)}\right] .
\end{aligned}
$$

Thus, from inequalities (41)-(44) we get $\operatorname{sgn}\left(\mathcal{R}_{1}^{Y}-1\right)=\operatorname{sgn}\left(H^{*}-\bar{H}\right)$. Similarly, one can show that $\operatorname{sgn}\left(\widehat{F}-F^{*}\right)=\operatorname{sgn}\left(H^{*}-\widehat{H}\right)=\operatorname{sgn}\left(\mathcal{R}_{1}^{Z}-1\right)$. Moreover, we have

$$
\digamma_{2}\left(S^{*}\right)-\digamma_{2}(\widehat{S})=\frac{c e^{\mu_{3} \tau_{3}}}{\theta}\left(\digamma_{3}\left(H^{*}\right)-\digamma_{3}(\widehat{H})\right),
$$

which gives us $\operatorname{sgn}\left(H^{*}-\widehat{H}\right)=\operatorname{sgn}\left(S^{*}-\widehat{S}\right)$. 
Proof of Theorem 2 Consider a function $\mathcal{U}_{n}\left(F_{n}, K_{n}, S_{n}, H_{n}, Y_{n}, Z_{n}\right)$ as follows:

$$
\begin{aligned}
\mathcal{U}_{n}= & \frac{1}{\phi(h)}\left[\gamma\left(F_{n}-F^{*}-\int_{F^{*}}^{F_{n}} \frac{\Lambda\left(F^{*}, H^{*}\right)}{\Lambda\left(\varsigma, H^{*}\right)} d \varsigma\right)+\frac{m e^{-\mu_{3} \tau_{3}}}{\alpha+m}\left(K_{n}-K^{*}-\int_{K^{*}}^{K_{n}} \frac{\digamma_{1}\left(K^{*}\right)}{\digamma_{1}(\varsigma)} d \varsigma\right)\right. \\
& +e^{-\mu_{3} \tau_{3}}\left(S_{n}-S^{*}-\int_{S^{*}}^{S_{n}} \frac{\digamma_{2}\left(S^{*}\right)}{\digamma_{2}(\varsigma)} d \varsigma\right) \\
& \left.+\frac{a}{\theta}\left(H_{n}-H^{*}-\int_{H^{*}}^{H_{n}} \frac{\digamma_{3}\left(H^{*}\right)}{\digamma_{3}(\varsigma)} d \varsigma\right)+\frac{a d}{\theta q} Y_{n}+\frac{\lambda e^{-\mu_{3} \tau_{3}}}{g} Z_{n}\right] \\
& +\frac{a c}{\theta} \digamma_{3}\left(H^{*}\right) G\left(\frac{\digamma_{3}\left(H_{n}\right)}{\digamma_{3}\left(H^{*}\right)}\right) \\
& \left.+\frac{m(1-\varepsilon) e^{-\mu_{1} \tau_{1}-\mu_{3} \tau_{3}}}{\alpha+m} \Lambda^{m}, F^{*}\right) \sum_{j=n-m_{1}}^{n-1} G\left(\frac{\Lambda\left(F_{j+1}, H_{j}\right)}{\Lambda\left(F^{*}, H^{*}\right)}\right) \\
& +\varepsilon e^{-\mu_{2} \tau_{2}-\mu_{3} \tau_{3}} \Lambda\left(F^{*}, H^{*}\right) \sum_{j=n-m_{2}}^{n-1} G\left(\frac{\Lambda\left(F_{j+1}, H_{j}\right)}{\Lambda\left(F^{*}, H^{*}\right)}\right) \\
& +a e^{-\mu_{3} \tau_{3}} \digamma_{2}\left(S^{*}\right) \sum_{j=n-m_{3}}^{n-1} G\left(\frac{\digamma_{2}\left(S_{j+1}\right)}{\digamma_{2}\left(S^{*}\right)}\right) .
\end{aligned}
$$

We have $\mathcal{U}_{n}\left(F_{n}, K_{n}, S_{n}, H_{n}, Y_{n}, Z_{n}\right)>0$ for all $F_{n}, K_{n}, S_{n}, H_{n}, Y_{n}, Z_{n}>0$; moreover, $\mathcal{U}_{n}\left(F^{*}, K^{*}\right.$, $\left.S^{*}, H^{*}, 0,0\right)=0$. We compute $\Delta \mathcal{U}_{n}=\mathcal{U}_{n+1}-\mathcal{U}_{n}$ as follows:

$$
\begin{aligned}
& \Delta \mathcal{U}_{n}=\frac{1}{\phi(h)}\left[\gamma\left(F_{n+1}-F^{*}-\int_{F^{*}}^{F_{n+1}} \frac{\Lambda\left(F^{*}, H^{*}\right)}{\Lambda\left(\varsigma, H^{*}\right)} d \varsigma\right)\right. \\
& +\frac{m e^{-\mu_{3} \tau_{3}}}{\alpha+m}\left(K_{n+1}-K^{*}-\int_{K^{*}}^{K_{n+1}} \frac{\digamma_{1}\left(K^{*}\right)}{\digamma_{1}(\varsigma)} d \varsigma\right) \\
& +e^{-\mu_{3} \tau_{3}}\left(S_{n+1}-S^{*}-\int_{S^{*}}^{S_{n+1}} \frac{\digamma_{2}\left(S^{*}\right)}{\digamma_{2}(\varsigma)} d \varsigma\right)+\frac{a}{\theta}\left(H_{n+1}-H^{*}-\int_{H^{*}}^{H_{n+1}} \frac{\digamma_{3}\left(H^{*}\right)}{\digamma_{3}(\varsigma)} d \varsigma\right) \\
& \left.+\frac{a d}{\theta q} Y_{n+1}+\frac{\lambda e^{-\mu_{3} \tau_{3}}}{g} Z_{n+1}\right]+\frac{a c}{\theta} \digamma_{3}\left(H^{*}\right) G\left(\frac{\digamma_{3}\left(H_{n+1}\right)}{\digamma_{3}\left(H^{*}\right)}\right) \\
& +\frac{m(1-\varepsilon) e^{-\mu_{1} \tau_{1}-\mu_{3} \tau_{3}}}{\alpha+m} \Lambda\left(F^{*}, H^{*}\right) \sum_{j=n-m_{1}+1}^{n} G\left(\frac{\Lambda\left(F_{j+1}, H_{j}\right)}{\Lambda\left(F^{*}, H^{*}\right)}\right) \\
& +\varepsilon e^{-\mu_{2} \tau_{2}-\mu_{3} \tau_{3}} \Lambda\left(F^{*}, H^{*}\right) \sum_{j=n-m_{2}+1}^{n} G\left(\frac{\Lambda\left(F_{j+1}, H_{j}\right)}{\Lambda\left(F^{*}, H^{*}\right)}\right) \\
& +a e^{-\mu_{3} \tau_{3}} \digamma_{2}\left(S^{*}\right) \sum_{j=n-m_{3}+1}^{n} G\left(\frac{\digamma_{2}\left(S_{j+1}\right)}{\digamma_{2}\left(S^{*}\right)}\right) \\
& -\frac{1}{\phi(h)}\left[\gamma\left(F_{n}-F^{*}-\int_{F^{*}}^{F_{n}} \frac{\Lambda\left(F^{*}, H^{*}\right)}{\Lambda\left(\varsigma, H^{*}\right)} d \varsigma\right)\right. \\
& +\frac{m e^{-\mu_{3} \tau_{3}}}{\alpha+m}\left(K_{n}-K^{*}-\int_{K^{*}}^{K_{n}} \frac{\digamma_{1}\left(K^{*}\right)}{\digamma_{1}(\varsigma)} d \varsigma\right) \\
& +e^{-\mu_{3} \tau_{3}}\left(S_{n}-S^{*}-\int_{S^{*}}^{S_{n}} \frac{\digamma_{2}\left(S^{*}\right)}{\digamma_{2}(\varsigma)} d \varsigma\right)
\end{aligned}
$$




$$
\begin{aligned}
& \left.+\frac{a}{\theta}\left(H_{n}-H^{*}-\int_{H^{*}}^{H_{n}} \frac{\digamma_{3}\left(H^{*}\right)}{\digamma_{3}(\varsigma)} d \varsigma\right)+\frac{a d}{\theta q} Y_{n}+\frac{\lambda e^{-\mu_{3} \tau_{3}}}{g} Z_{n}\right] \\
& -\frac{a c}{\theta} \digamma_{3}\left(H^{*}\right) G\left(\frac{\digamma_{3}\left(H_{n}\right)}{\digamma_{3}\left(H^{*}\right)}\right) \\
& -\frac{m(1-\varepsilon) e^{-\mu_{1} \tau_{1}-\mu_{3} \tau_{3}}}{\alpha+m} \Lambda\left(F^{*}, H^{*}\right) \sum_{j=n-m_{1}}^{n-1} G\left(\frac{\Lambda\left(F_{j+1}, H_{j}\right)}{\Lambda\left(F^{*}, H^{*}\right)}\right) \\
& -\varepsilon e^{-\mu_{2} \tau_{2}-\mu_{3} \tau_{3}} \Lambda\left(F^{*}, H^{*}\right) \sum_{j=n-m_{2}}^{n-1} G\left(\frac{\Lambda\left(F_{j+1}, H_{j}\right)}{\Lambda\left(F^{*}, H^{*}\right)}\right) \\
& -a e^{-\mu_{3} \tau_{3}} \digamma_{2}\left(S^{*}\right) \sum_{j=n-m_{3}}^{n-1} G\left(\frac{\digamma_{2}\left(S_{j+1}\right)}{\digamma_{2}\left(S^{*}\right)}\right) \text {, } \\
& \Delta \mathcal{U}_{n}=\frac{1}{\phi(h)}\left[\gamma\left(F_{n+1}-F_{n}-\int_{F_{n}}^{F_{n+1}} \frac{\Lambda\left(F^{*}, H^{*}\right)}{\Lambda\left(\varsigma, H^{*}\right)} d \varsigma\right)\right. \\
& +\frac{m e^{-\mu_{3} \tau_{3}}}{\alpha+m}\left(K_{n+1}-K_{n}-\int_{K_{n}}^{K_{n+1}} \frac{\digamma_{1}\left(K^{*}\right)}{\digamma_{1}(\varsigma)} d \varsigma\right) \\
& +e^{-\mu_{3} \tau_{3}}\left(S_{n+1}-S_{n}-\int_{S_{n}}^{S_{n+1}} \frac{\digamma_{2}\left(S^{*}\right)}{\digamma_{2}(\varsigma)} d \varsigma\right)+\frac{a}{\theta}\left(H_{n+1}-H_{n}-\int_{H_{n}}^{H_{n+1}} \frac{\digamma_{3}\left(H^{*}\right)}{\digamma_{3}(\varsigma)} d \varsigma\right) \\
& \left.+\frac{a d}{\theta q}\left(Y_{n+1}-Y_{n}\right)+\frac{\lambda e^{-\mu_{3} \tau_{3}}}{g}\left(Z_{n+1}-Z_{n}\right)\right] \\
& +\frac{a c}{\theta} \digamma_{3}\left(H^{*}\right)\left[G\left(\frac{\digamma_{3}\left(H_{n+1}\right)}{\digamma_{3}\left(H^{*}\right)}\right)-G\left(\frac{\digamma_{3}\left(H_{n}\right)}{\digamma_{3}\left(H^{*}\right)}\right)\right] \\
& +\frac{m(1-\varepsilon) e^{-\mu_{1} \tau_{1}-\mu_{3} \tau_{3}}}{\alpha+m} \Lambda\left(F^{*}, H^{*}\right)\left[G\left(\frac{\Lambda\left(F_{n+1}, H_{n}\right)}{\Lambda\left(F^{*}, H^{*}\right)}\right)\right. \\
& \left.-G\left(\frac{\Lambda\left(F_{n-m_{1}+1}, H_{n-m_{1}}\right)}{\Lambda\left(F^{*}, H^{*}\right)}\right)\right] \\
& +\varepsilon e^{-\mu_{2} \tau_{2}-\mu_{3} \tau_{3}} \Lambda\left(F^{*}, H^{*}\right)\left[G\left(\frac{\Lambda\left(F_{n+1}, H_{n}\right)}{\Lambda\left(F^{*}, H^{*}\right)}\right)-G\left(\frac{\Lambda\left(F_{n-m_{2}+1}, H_{n-m_{2}}\right)}{\Lambda\left(F^{*}, H^{*}\right)}\right)\right] \\
& +a e^{-\mu_{3} \tau_{3}} \digamma_{2}\left(S^{*}\right)\left[G\left(\frac{\digamma_{2}\left(S_{n+1}\right)}{\digamma_{2}\left(S^{*}\right)}\right)-G\left(\frac{\digamma_{2}\left(S_{n-m_{3}+1}\right)}{\digamma_{2}\left(S^{*}\right)}\right)\right] \text {. }
\end{aligned}
$$

We have

$$
\begin{aligned}
\left(1-\frac{\Lambda\left(F^{*}, H^{*}\right)}{\Lambda\left(F_{n}, H^{*}\right)}\right)\left(F_{n+1}-F_{n}\right) & \leq F_{n+1}-F_{n}-\int_{F_{n}}^{F_{n+1}} \frac{\Lambda\left(F^{*}, H^{*}\right)}{\Lambda\left(\varsigma, H^{*}\right)} d \varsigma \\
& \leq\left(1-\frac{\Lambda\left(F^{*}, H^{*}\right)}{\Lambda\left(F_{n+1}, H^{*}\right)}\right)\left(F_{n+1}-F_{n}\right), \\
\left(1-\frac{\digamma_{i}\left(\rho^{*}\right)}{\digamma_{i}\left(\rho_{n}\right)}\right)\left(\rho_{n+1}-\rho_{n}\right) & \leq \rho_{n+1}-\rho_{n}-\int_{\rho_{n}}^{\rho_{n+1}} \frac{\digamma_{i}\left(\rho^{*}\right)}{\digamma_{i}(\varsigma)} d \varsigma \\
& \leq\left(1-\frac{\digamma_{i}\left(\rho^{*}\right)}{\digamma_{i}\left(\rho_{n+1}\right)}\right)\left(\rho_{n+1}-\rho_{n}\right),
\end{aligned}
$$

$i=1, \ldots, 5, \rho^{*} \in\left\{K^{*}, S^{*}, H^{*}\right\}$. 
Then

$$
\begin{aligned}
\Delta \mathcal{U}_{n} \leq & \frac{1}{\phi(h)}\left[\gamma\left(1-\frac{\Lambda\left(F^{*}, H^{*}\right)}{\Lambda\left(F_{n+1}, H^{*}\right)}\right)\left(F_{n+1}-F_{n}\right)+\frac{m e^{-\mu_{3} \tau_{3}}}{\alpha+m}\left(1-\frac{\digamma_{1}\left(K^{*}\right)}{\digamma_{1}\left(K_{n+1}\right)}\right)\left(K_{n+1}-K_{n}\right)\right. \\
& +e^{-\mu_{3} \tau_{3}}\left(1-\frac{\digamma_{2}\left(S^{*}\right)}{\digamma_{2}\left(S_{n+1}\right)}\right)\left(S_{n+1}-S_{n}\right)+\frac{a}{\theta}\left(1-\frac{\digamma_{3}\left(H^{*}\right)}{\digamma_{3}\left(H_{n+1}\right)}\right)\left(H_{n+1}-H_{n}\right) \\
& +\frac{a d}{\theta q}\left(Y_{n+1}-Y_{n}\right) \\
& \left.+\frac{\lambda e^{-\mu_{3} \tau_{3}}}{g}\left(Z_{n+1}-Z_{n}\right)\right]+\frac{a c}{\theta} \digamma_{3}\left(H^{*}\right)\left[\frac{\digamma_{3}\left(H_{n+1}\right)}{\digamma_{3}\left(H^{*}\right)}-\frac{\digamma_{3}\left(H_{n}\right)}{\digamma_{3}\left(H^{*}\right)}+\ln \left(\frac{\digamma_{3}\left(H_{n}\right)}{\digamma_{3}\left(H_{n+1}\right)}\right)\right] \\
& +\frac{m(1-\varepsilon) e^{-\mu_{1} \tau_{1}-\mu_{3} \tau_{3}}}{\alpha+m} \Lambda\left(F^{*}, H^{*}\right)\left[\frac{\Lambda\left(F_{n+1}, H_{n}\right)}{\Lambda\left(F^{*}, H^{*}\right)}-\frac{\Lambda\left(F_{n-m_{1}+1}, H_{n-m_{1}}\right)}{\Lambda\left(F^{*}, H^{*}\right)}\right. \\
& \left.+\ln \left(\frac{\Lambda\left(F_{n-m_{1}+1}, H_{n-m_{1}}\right)}{\Lambda\left(F_{n+1}, H_{n}\right)}\right)\right] \\
& +\varepsilon e^{-\mu_{2} \tau_{2}-\mu_{3} \tau_{3}} \Lambda\left(F^{*}, H^{*}\right)\left[\frac{\Lambda\left(F_{n+1}, H_{n}\right)}{\Lambda\left(F^{*}, H^{*}\right)}-\frac{\Lambda\left(F_{n-m_{2}+1}, H_{n-m_{2}}\right)}{\Lambda\left(F^{*}, H^{*}\right)}\right. \\
& \left.+\ln \left(\frac{\Lambda\left(F_{n-m_{2}+1}, H_{n-m_{2}}\right)}{\Lambda\left(F_{n+1}, H_{n}\right)}\right)\right] \\
& +a e^{-\mu_{3} \tau_{3}} \digamma_{2}\left(S^{*}\right)\left[\frac{\digamma_{2}\left(S_{n+1}\right)}{\digamma_{2}\left(S^{*}\right)}-\frac{\digamma_{2}\left(S_{n-m_{3}+1}\right)}{\digamma_{2}\left(S^{*}\right)}+\ln \left(\frac{\digamma_{2}\left(S_{n-m_{3}+1}\right)}{\digamma_{2}\left(S_{n+1}\right)}\right)\right] .
\end{aligned}
$$

From Eqs. (11)-(16) we have

$$
\begin{aligned}
\Delta \mathcal{U}_{n} \leq & \gamma\left(1-\frac{\Lambda\left(F^{*}, H^{*}\right)}{\Lambda\left(F_{n+1}, H^{*}\right)}\right)\left(\Theta\left(F_{n+1}\right)-\Lambda\left(F_{n+1}, H_{n}\right)\right) \\
& +\frac{m e^{-\mu_{3} \tau_{3}}}{\alpha+m}\left(1-\frac{\digamma_{1}\left(K^{*}\right)}{\digamma_{1}\left(K_{n+1}\right)}\right)\left[(1-\varepsilon) e^{-\mu_{1} \tau_{1}} \Lambda\left(F_{n-m_{1}+1}, H_{n-m_{1}}\right)\right. \\
& \left.-(\alpha+m) \digamma_{1}\left(K_{n+1}\right)\right] \\
& +e^{-\mu_{3} \tau_{3}}\left(1-\frac{\digamma_{2}\left(S^{*}\right)}{\digamma_{2}\left(S_{n+1}\right)}\right)\left[\varepsilon e^{-\mu_{2} \tau_{2}} \Lambda\left(F_{n-m_{2}+1}, H_{n-m_{2}}\right)+m \digamma_{1}\left(K_{n+1}\right)-a \digamma_{2}\left(S_{n+1}\right)\right. \\
& \left.-\lambda \digamma_{2}\left(S_{n+1}\right) \digamma_{5}\left(Z_{n+1}\right)\right] \\
& +\frac{a}{\theta}\left(1-\frac{\digamma_{3}\left(H^{*}\right)}{\digamma_{3}\left(H_{n+1}\right)}\right)\left[\theta e^{-\mu_{3} \tau_{3}} \digamma_{2}\left(S_{n-m_{3}+1}\right)-c \digamma_{3}\left(H_{n+1}\right)-d \digamma_{3}\left(H_{n+1}\right) \digamma_{4}\left(Y_{n+1}\right)\right] \\
& +\frac{a d}{\theta q}\left[q \digamma_{3}\left(H_{n+1}\right) \digamma_{4}\left(Y_{n+1}\right)-\eta \digamma_{4}\left(Y_{n+1}\right)\right] \\
& +\frac{\lambda e^{-\mu_{3} \tau_{3}}}{g}\left[g \digamma_{2}\left(S_{n+1}\right) \digamma_{5}\left(Z_{n+1}\right)-\xi \digamma_{5}\left(Z_{n+1}\right)\right] \\
& +\frac{a c}{\theta} \digamma_{3}\left(H^{*}\right)\left[\frac{\digamma_{3}\left(H_{n+1}\right)}{\digamma_{3}\left(H^{*}\right)}-\frac{\digamma_{3}\left(H_{n}\right)}{\digamma_{3}\left(H^{*}\right)}+\ln \left(\frac{\digamma_{3}\left(H_{n}\right)}{\digamma_{3}\left(H_{n+1}\right)}\right)\right] \\
& +\frac{m(1-\varepsilon) e^{-\mu_{1} \tau_{1}-\mu_{3} \tau_{3}}}{\alpha+m}\left(F^{*}, H^{*}\right)\left[\frac{\Lambda\left(F_{n+1}, H_{n}\right)}{\Lambda\left(F^{*}, H^{*}\right)}-\frac{\Lambda\left(F_{n-m_{1}+1}, H_{n-m_{1}}\right)}{\Lambda\left(F^{*}, H^{*}\right)}\right. \\
& \left.+\ln \left(\frac{\Lambda\left(F_{n-m_{1}+1}, H_{n-m_{1}}\right)}{\Lambda\left(F_{n+1}, H_{n}\right)}\right)\right]
\end{aligned}
$$




$$
\begin{aligned}
& +\varepsilon e^{-\mu_{2} \tau_{2}-\mu_{3} \tau_{3}} \Lambda\left(F^{*}, H^{*}\right)\left[\frac{\Lambda\left(F_{n+1}, H_{n}\right)}{\Lambda\left(F^{*}, H^{*}\right)}-\frac{\Lambda\left(F_{n-m_{2}+1}, H_{n-m_{2}}\right)}{\Lambda\left(F^{*}, H^{*}\right)}\right. \\
& \left.+\ln \left(\frac{\Lambda\left(F_{n-m_{2}+1}, H_{n-m_{2}}\right)}{\Lambda\left(F_{n+1}, H_{n}\right)}\right)\right] \\
& +a e^{-\mu_{3} \tau_{3}} \digamma_{2}\left(S^{*}\right)\left[\frac{\digamma_{2}\left(S_{n+1}\right)}{\digamma_{2}\left(S^{*}\right)}-\frac{\digamma_{2}\left(S_{n-m_{3}+1}\right)}{\digamma_{2}\left(S^{*}\right)}+\ln \left(\frac{\digamma_{2}\left(S_{n-m_{3}+1}\right)}{\digamma_{2}\left(S_{n+1}\right)}\right)\right] .
\end{aligned}
$$

Collecting terms of Eq. (47), we get

$$
\begin{aligned}
& \Delta \mathcal{U}_{n} \leq \gamma\left(1-\frac{\Lambda\left(F^{*}, H^{*}\right)}{\Lambda\left(F_{n+1}, H^{*}\right)}\right)\left(\Theta\left(F_{n+1}\right)-\Theta\left(F^{*}\right)\right)+\gamma \Theta\left(F^{*}\right)\left(1-\frac{\Lambda\left(F^{*}, H^{*}\right)}{\Lambda\left(F_{n+1}, H^{*}\right)}\right) \\
& +\gamma \frac{\Lambda\left(F^{*}, H^{*}\right)}{\Lambda\left(F_{n+1}, H^{*}\right)} \Lambda\left(F_{n+1}, H_{n}\right)-\frac{m(1-\varepsilon)}{\alpha+m} e^{-\mu_{1} \tau_{1}-\mu_{3} \tau_{3}} \Lambda\left(F_{n-m_{1}+1}, H_{n-m_{1}}\right) \frac{\digamma_{1}\left(K^{*}\right)}{\digamma_{1}\left(K_{n+1}\right)} \\
& +m e^{-\mu_{3} \tau_{3}} \digamma_{1}\left(K^{*}\right)-\varepsilon e^{-\mu_{2} \tau_{2}-\mu_{3} \tau_{3}} \Lambda\left(F_{n-m_{2}+1}, H_{n-m_{2}}\right) \frac{\digamma_{2}\left(S^{*}\right)}{\digamma_{2}\left(S_{n+1}\right)} \\
& -m e^{-\mu_{3} \tau_{3}} \digamma_{1}\left(K_{n+1}\right) \frac{\digamma_{2}\left(S^{*}\right)}{\digamma_{2}\left(S_{n+1}\right)} \\
& +a e^{-\mu_{3} \tau_{3}} \digamma_{2}\left(S^{*}\right)+\lambda e^{-\mu_{3} \tau_{3}} \digamma_{2}\left(S^{*}\right) \digamma_{5}\left(Z_{n+1}\right)-a e^{-\mu_{3} \tau_{3}} \digamma_{2}\left(S_{n-m_{3}+1}\right) \frac{\digamma_{3}\left(H^{*}\right)}{\digamma_{3}\left(H_{n+1}\right)} \\
& +\frac{a c}{\theta} \digamma_{3}\left(H^{*}\right) \\
& +\frac{a d}{\theta} \digamma_{3}\left(H^{*}\right) \digamma_{4}\left(Y_{n+1}\right)-\frac{a d \eta}{\theta q} \digamma_{4}\left(Y_{n+1}\right)-\frac{\lambda \xi e^{-\mu_{3} \tau_{3}}}{g} \digamma_{5}\left(Z_{n+1}\right)-\frac{a c}{\theta} \digamma_{3}\left(H_{n}\right) \\
& +\frac{a c}{\theta} \digamma_{3}\left(H^{*}\right) \ln \left(\frac{\digamma_{3}\left(H_{n}\right)}{\digamma_{3}\left(H_{n+1}\right)}\right) \\
& +\frac{m(1-\varepsilon) e^{-\mu_{1} \tau_{1}-\mu_{3} \tau_{3}}}{\alpha+m} \Lambda\left(F^{*}, H^{*}\right) \ln \left(\frac{\Lambda\left(F_{n-m_{1}+1}, H_{n-m_{1}}\right)}{\Lambda\left(F_{n+1}, H_{n}\right)}\right) \\
& +\varepsilon e^{-\mu_{2} \tau_{2}-\mu_{3} \tau_{3}} \Lambda\left(F^{*}, H^{*}\right) \ln \left(\frac{\Lambda\left(F_{n-m_{2}+1}, H_{n-m_{2}}\right)}{\Lambda\left(F_{n+1}, H_{n}\right)}\right) \\
& +a e^{-\mu_{3} \tau_{3}} \digamma_{2}\left(S^{*}\right) \ln \left(\frac{\digamma_{2}\left(S_{n-m_{3}+1}\right)}{\digamma_{2}\left(S_{n+1}\right)}\right) \text {. }
\end{aligned}
$$

Using the conditions of $Q^{*}$

$$
\left\{\begin{array}{l}
\Theta\left(F^{*}\right)=\Lambda\left(F^{*}, H^{*}\right), \\
(1-\varepsilon) e^{-\mu_{1} \tau_{1}} \Lambda\left(F^{*}, H^{*}\right)=(\alpha+m) \digamma_{1}\left(K^{*}\right), \\
\varepsilon e^{-\mu_{2} \tau_{2}-\mu_{3} \tau_{3}} \Lambda\left(F^{*}, H^{*}\right)+m e^{-\mu_{3} \tau_{3}} \digamma_{1}\left(K^{*}\right)=\gamma \Lambda\left(F^{*}, H^{*}\right)=a e^{-\mu_{3} \tau_{3}} \digamma_{2}\left(S^{*}\right), \\
\theta e^{-\mu_{3} \tau_{3}} \digamma_{2}\left(S^{*}\right)=c \digamma_{3}\left(H^{*}\right),
\end{array}\right.
$$

we get

$$
\begin{aligned}
\Delta \mathcal{U}_{n} \leq & \gamma\left(1-\frac{\Lambda\left(F^{*}, H^{*}\right)}{\Lambda\left(F_{n+1}, H^{*}\right)}\right)\left(\Theta\left(F_{n+1}\right)-\Theta\left(F^{*}\right)\right) \\
& +\gamma \Lambda\left(F^{*}, H^{*}\right)\left(1-\frac{\Lambda\left(F^{*}, H^{*}\right)}{\Lambda\left(F_{n+1}, H^{*}\right)}\right)
\end{aligned}
$$




$$
\begin{aligned}
& +\gamma \Lambda\left(F^{*}, H^{*}\right) \frac{\Lambda\left(F_{n+1}, H_{n}\right)}{\Lambda\left(F_{n+1}, H^{*}\right)} \\
& -\frac{m(1-\varepsilon)}{\alpha+m} e^{-\mu_{1} \tau_{1}-\mu_{3} \tau_{3}} \Lambda\left(F^{*}, H^{*}\right) \frac{\digamma_{1}\left(K^{*}\right) \Lambda\left(F_{n-m_{1}+1}, H_{n-m_{1}}\right)}{\digamma_{1}\left(K_{n+1}\right) \Lambda\left(F^{*}, H^{*}\right)} \\
& +\frac{m(1-\varepsilon)}{\alpha+m} e^{-\mu_{1} \tau_{1}-\mu_{3} \tau_{3}} \Lambda\left(F^{*}, H^{*}\right) \\
& -\varepsilon e^{-\mu_{2} \tau_{2}-\mu_{3} \tau_{3}} \Lambda\left(F^{*}, H^{*}\right) \frac{\digamma_{2}\left(S^{*}\right) \Lambda\left(F_{n-m_{2}+1}, H_{n-m_{2}}\right)}{\digamma_{2}\left(S_{n+1}\right) \Lambda\left(F^{*}, H^{*}\right)} \\
& -\frac{m(1-\varepsilon)}{\alpha+m} e^{-\mu_{1} \tau_{1}-\mu_{3} \tau_{3}} \Lambda\left(F^{*}, H^{*}\right) \frac{\digamma_{2}\left(S^{*}\right) \digamma_{1}\left(K_{n+1}\right)}{\digamma_{2}\left(S_{n+1}\right) \digamma_{1}\left(K^{*}\right)}+\gamma \Lambda\left(F^{*}, H^{*}\right) \\
& -\gamma \Lambda\left(F^{*}, H^{*}\right) \frac{\digamma_{3}\left(H^{*}\right) \digamma_{2}\left(S_{n-m_{3}+1}\right)}{\digamma_{3}\left(H_{n+1}\right) \digamma_{2}\left(S^{*}\right)}+\gamma \Lambda\left(F^{*}, H^{*}\right) \\
& +\frac{a d}{\theta}\left(\digamma_{3}\left(H^{*}\right)-\frac{\eta}{q}\right) \digamma_{4}\left(Y_{n+1}\right) \\
& +\lambda e^{-\mu_{3} \tau_{3}}\left(\digamma_{2}\left(S^{*}\right)-\frac{\xi}{g}\right) \digamma_{5}\left(Z_{n+1}\right)-\gamma \Lambda\left(F^{*}, H^{*}\right) \frac{\digamma_{3}\left(H_{n}\right)}{\digamma_{3}\left(H^{*}\right)} \\
& +\gamma \Lambda\left(F^{*}, H^{*}\right) \ln \left(\frac{\digamma_{3}\left(H_{n}\right)}{\digamma_{3}\left(H_{n+1}\right)}\right) \\
& +\frac{m(1-\varepsilon) e^{-\mu_{1} \tau_{1}-\mu_{3} \tau_{3}}}{\alpha+m} \Lambda\left(F^{*}, H^{*}\right) \ln \left(\frac{\Lambda\left(F_{n-m_{1}+1}, H_{n-m_{1}}\right)}{\Lambda\left(F_{n+1}, H_{n}\right)}\right) \\
& +\varepsilon e^{-\mu_{2} \tau_{2}-\mu_{3} \tau_{3}} \Lambda\left(F^{*}, H^{*}\right) \ln \left(\frac{\Lambda\left(F_{n-m_{2}+1}, H_{n-m_{2}}\right)}{\Lambda\left(F_{n+1}, H_{n}\right)}\right) \\
& +\gamma \Lambda\left(F^{*}, H^{*}\right) \ln \left(\frac{\digamma_{2}\left(S_{n-m_{3}+1}\right)}{\digamma_{2}\left(S_{n+1}\right)}\right) \text {. }
\end{aligned}
$$

It follows that

$$
\begin{aligned}
& \Delta \mathcal{U}_{n} \leq \gamma\left(1-\frac{\Lambda\left(F^{*}, H^{*}\right)}{\Lambda\left(F_{n+1}, H^{*}\right)}\right)\left(\Theta\left(F_{n+1}\right)-\Theta\left(F^{*}\right)\right) \\
& +\frac{m(1-\varepsilon) e^{-\mu_{1} \tau_{1}-\mu_{3} \tau_{3}}}{\alpha+m} \Lambda\left(F^{*}, H^{*}\right) \\
& \times\left[5-\frac{\Lambda\left(F^{*}, H^{*}\right)}{\Lambda\left(F_{n+1}, H^{*}\right)}-\frac{\digamma_{1}\left(K^{*}\right) \Lambda\left(F_{n-m_{1}+1}, H_{n-m_{1}}\right)}{\digamma_{1}\left(K_{n+1}\right) \Lambda\left(F^{*}, H^{*}\right)}\right. \\
& -\frac{\digamma_{2}\left(S^{*}\right) \digamma_{1}\left(K_{n+1}\right)}{\digamma_{2}\left(S_{n+1}\right) \digamma_{1}\left(K^{*}\right)}-\frac{\digamma_{3}\left(H^{*}\right) \digamma_{2}\left(S_{n-m_{3}+1}\right)}{\digamma_{3}\left(H_{n+1}\right) \digamma_{2}\left(S^{*}\right)}-\frac{\Lambda\left(F_{n+1}, H^{*}\right) \digamma_{3}\left(H_{n}\right)}{\Lambda\left(F_{n+1}, H_{n}\right) \digamma_{3}\left(H^{*}\right)} \\
& \left.+\ln \left(\frac{\digamma_{3}\left(H_{n}\right) \Lambda\left(F_{n-m_{1}+1}, H_{n-m_{1}}\right) \digamma_{2}\left(S_{n-m_{3}+1}\right)}{\digamma_{3}\left(H_{n+1}\right) \Lambda\left(F_{n+1}, H_{n}\right) \digamma_{2}\left(S_{n+1}\right)}\right)\right] \\
& +\varepsilon e^{-\mu_{2} \tau_{2}-\mu_{3} \tau_{3}} \Lambda\left(F^{*}, H^{*}\right)\left[4-\frac{\Lambda\left(F^{*}, H^{*}\right)}{\Lambda\left(F_{n+1}, H^{*}\right)}-\frac{\digamma_{2}\left(S^{*}\right) \Lambda\left(F_{n-m_{2}+1}, H_{n-m_{2}}\right)}{\digamma_{2}\left(S_{n+1}\right) \Lambda\left(F^{*}, H^{*}\right)}\right. \\
& -\frac{\digamma_{3}\left(H^{*}\right) \digamma_{2}\left(S_{n-m_{3}+1}\right)}{\digamma_{3}\left(H_{n+1}\right) \digamma_{2}\left(S^{*}\right)}-\frac{\Lambda\left(F_{n+1}, H^{*}\right) \digamma_{3}\left(H_{n}\right)}{\Lambda\left(F_{n+1}, H_{n}\right) \digamma_{3}\left(H^{*}\right)} \\
& \left.+\ln \left(\frac{\digamma_{3}\left(H_{n}\right) \Lambda\left(F_{n-m_{2}+1}, H_{n-m_{2}}\right) \digamma_{2}\left(S_{n-m_{3}+1}\right)}{\digamma_{3}\left(H_{n+1}\right) \Lambda\left(F_{n+1}, H_{n}\right) \digamma_{2}\left(S_{n+1}\right)}\right)\right] \\
& +\frac{a d}{\theta}\left(\digamma_{3}\left(H^{*}\right)-\digamma_{3}(\bar{H})\right) \digamma_{4}\left(Y_{n+1}\right)+\lambda e^{-\mu_{3} \tau_{3}}\left(\digamma_{2}\left(S^{*}\right)-\digamma_{2}(\widehat{S})\right) \digamma_{5}\left(S_{n+1}\right)
\end{aligned}
$$




$$
\begin{aligned}
& +\gamma \Lambda\left(F^{*}, H^{*}\right)\left[-1+\frac{\Lambda\left(F_{n+1}, H^{*}\right) \digamma_{3}\left(H_{n}\right)}{\Lambda\left(F_{n+1}, H_{n}\right) \digamma_{3}\left(H^{*}\right)}+\frac{\Lambda\left(F_{n+1}, H_{n}\right)}{\Lambda\left(F_{n+1}, H^{*}\right)}-\frac{\digamma_{3}\left(H_{n}\right)}{\digamma_{3}\left(H^{*}\right)}\right] \\
& =\gamma\left(1-\frac{\Lambda\left(F^{*}, H^{*}\right)}{\Lambda\left(F_{n+1}, H^{*}\right)}\right)\left(\Theta\left(F_{n+1}\right)-\Theta\left(F^{*}\right)\right) \\
& -\frac{m(1-\varepsilon) e^{-\mu_{1} \tau_{1}-\mu_{3} \tau_{3}}}{\alpha+m} \Lambda\left(F^{*}, H^{*}\right)\left[G\left(\frac{\Lambda\left(F^{*}, H^{*}\right)}{\Lambda\left(F_{n+1}, H^{*}\right)}\right)\right. \\
& +G\left(\frac{\digamma_{1}\left(K^{*}\right) \Lambda\left(F_{n-m_{1}+1}, H_{n-m_{1}}\right)}{\digamma_{1}\left(K_{n+1}\right) \Lambda\left(F^{*}, H^{*}\right)}\right) \\
& +G\left(\frac{\digamma_{2}\left(S^{*}\right) \digamma_{1}\left(K_{n+1}\right)}{\digamma_{2}\left(S_{n+1}\right) \digamma_{1}\left(K^{*}\right)}\right)+G\left(\frac{\digamma_{3}\left(H^{*}\right) \digamma_{2}\left(S_{n-m_{3}+1}\right)}{\digamma_{3}\left(H_{n+1}\right) \digamma_{2}\left(S^{*}\right)}\right) \\
& \left.+G\left(\frac{\Lambda\left(F_{n+1}, H^{*}\right) \digamma_{3}\left(H_{n}\right)}{\Lambda\left(F_{n+1}, H_{n}\right) \digamma_{3}\left(H^{*}\right)}\right)\right] \\
& -\varepsilon e^{-\mu_{2} \tau_{2}-\mu_{3} \tau_{3}} \Lambda\left(F^{*}, H^{*}\right)\left[G\left(\frac{\Lambda\left(F^{*}, H^{*}\right)}{\Lambda\left(F_{n+1}, H^{*}\right)}\right)\right. \\
& +G\left(\frac{\digamma_{2}\left(S^{*}\right) \Lambda\left(F_{n-m_{2}+1}, H_{n-m_{2}}\right)}{\digamma_{2}\left(S_{n+1}\right) \Lambda\left(F^{*}, H^{*}\right)}\right) \\
& \left.+G\left(\frac{\digamma_{3}\left(H^{*}\right) \digamma_{2}\left(S_{n-m_{3}+1}\right)}{\digamma_{3}\left(H_{n+1}\right) \digamma_{2}\left(S^{*}\right)}\right)+G\left(\frac{\Lambda\left(F_{n+1}, H^{*}\right) \digamma_{3}\left(H_{n}\right)}{\Lambda\left(F_{n+1}, H_{n}\right) \digamma_{3}\left(H^{*}\right)}\right)\right] \\
& +\frac{a d}{\theta}\left(\digamma_{3}\left(H^{*}\right)-\digamma_{3}(\bar{H})\right) \digamma_{4}\left(Y_{n+1}\right)+\lambda e^{-\mu_{3} \tau_{3}}\left(\digamma_{2}\left(S^{*}\right)-\digamma_{2}(\widehat{S})\right) \digamma_{5}\left(Z_{n+1}\right) \\
& +\gamma \Lambda\left(F^{*}, H^{*}\right)\left(1-\frac{\Lambda\left(F_{n+1}, H^{*}\right)}{\Lambda\left(F_{n+1}, H_{n}\right)}\right)\left(\frac{\Lambda\left(F_{n+1}, H_{n}\right)}{\Lambda\left(F_{n+1}, H^{*}\right)}-\frac{\digamma_{3}\left(H_{n}\right)}{\digamma_{3}\left(H^{*}\right)}\right) .
\end{aligned}
$$

Conditions C1-C4 imply that the first and last terms of Eq. (49) are less than or equal to zero. If $\mathcal{R}_{1}^{Y} \leq 1$, then from Lemma 3 we have $H^{*} \leq \bar{H}$ and from Condition C3 we get $\digamma_{3}\left(H^{*}\right) \leq \digamma_{3}(\bar{H})$. Moreover, if $\mathcal{R}_{1}^{Z} \leq 1$, then $\digamma_{2}\left(S^{*}\right) \leq \digamma_{2}(\widehat{S})$. Therefore, $\Delta \mathcal{U}_{n} \leq 0$, and thus $\mathcal{U}_{n}$ is a monotone decreasing sequence. Since $\mathcal{U}_{n} \geq 0$, then there is a limit $\lim _{n \rightarrow \infty} \mathcal{U}_{n} \geq 0$. Therefore, $\lim _{n \rightarrow \infty} \Delta \mathcal{U}_{n}=0$, which implies that $\lim _{n \rightarrow \infty} F_{n}=F^{*}$, $\lim _{n \rightarrow \infty} K_{n}=K^{*}, \lim _{n \rightarrow \infty} S_{n}=S^{*}, \lim _{n \rightarrow \infty} H_{n}=H^{*}, \lim _{n \rightarrow \infty} Y_{n}=0$, and $\lim _{n \rightarrow \infty} Z_{n}=0$. We have four cases as follows:

- $\mathcal{R}_{1}^{Y}=1, \mathcal{R}_{1}^{Z}=1$, then from Eq. (13)

$$
\begin{aligned}
0= & \varepsilon e^{-\mu_{2} \tau_{2}} \Lambda\left(F^{*}, H^{*}\right)+m \digamma_{1}\left(K^{*}\right)-a \digamma_{2}\left(S^{*}\right) \\
& -\lambda \digamma_{2}\left(S^{*}\right) \lim _{n \rightarrow \infty} \digamma_{5}\left(Z_{n+1}\right) .
\end{aligned}
$$

Using equilibrium condition (48), we get $\lim _{n \rightarrow \infty} Z_{n}=0$. Moreover, from Eq. (14) we have

$$
0=\theta e^{-\mu_{3} \tau_{3}} \digamma_{2}\left(S^{*}\right)-c \digamma_{3}\left(H^{*}\right)-d \digamma_{3}\left(H^{*}\right) \lim _{n \rightarrow \infty} \digamma_{4}\left(Y_{n+1}\right) .
$$

From Eq. (48) we get $\lim _{n \rightarrow \infty} Y_{n}=0$.

- $\mathcal{R}_{1}^{Y}=1, \mathcal{R}_{1}^{Z}<1$, and $\lim _{n \rightarrow \infty} Z_{n}=0$. From Eq. (51) we get $\lim _{n \rightarrow \infty} Y_{n}=0$.

- $\mathcal{R}_{1}^{Y}<1, \mathcal{R}_{1}^{Z}=1, \lim _{n \rightarrow \infty} Y_{n}=0$. From Eq. (50) we get $\lim _{n \rightarrow \infty} Z_{n}=0$. 
- $\mathcal{R}_{1}^{Y}<1, \mathcal{R}_{1}^{Z}<1, \lim _{n \rightarrow \infty} Y_{n}=0$, and $\lim _{n \rightarrow \infty} Z_{n}=0$. It follows that if $\mathcal{R}_{1}^{Z} \leq 1$ and $\mathcal{R}_{1}^{Y} \leq 1$, then $\lim _{n \rightarrow \infty} F_{n}=F^{*}, \lim _{n \rightarrow \infty} K_{n}=K^{*}, \lim _{n \rightarrow \infty} S_{n}=S^{*}, \lim _{n \rightarrow \infty} H_{n}=H^{*}$, $\lim _{n \rightarrow \infty} Y_{n}=0$, and $\lim _{n \rightarrow \infty} Z_{n}=0$. Then $Q^{*}$ is G.A.S.

Proof of Theorem 3 Define $\mathcal{W}_{n}\left(F_{n}, K_{n}, S_{n}, H_{n}, Y_{n}, Z_{n}\right)$

$$
\begin{aligned}
\mathcal{W}_{n}= & \frac{1}{\phi(h)}\left[\gamma\left(F_{n}-\bar{F}-\int_{\bar{F}}^{F_{n}} \frac{\Lambda(\bar{F}, \bar{H})}{\Lambda(\varsigma, \bar{H})} d \varsigma\right)+\frac{m e^{-\mu_{3} \tau_{3}}}{\alpha+m}\left(K_{n}-\bar{K}-\int_{\bar{K}}^{K_{n}} \frac{\digamma_{1}(\bar{K})}{\digamma_{1}(\varsigma)} d \varsigma\right)\right. \\
& +e^{-\mu_{3} \tau_{3}}\left(S_{n}-\bar{S}-\int_{\bar{S}}^{S_{n}} \frac{\digamma_{2}(\bar{S})}{\digamma_{2}(\varsigma)} d \varsigma\right)+\frac{a}{\theta}\left(H_{n}-\bar{H}-\int_{\bar{H}}^{H_{n}} \frac{\digamma_{3}(\bar{H})}{\digamma_{3}(\varsigma)} d \varsigma\right) \\
& \left.+\frac{a d}{\theta q}\left(Y_{n}-\bar{Y}-\int_{\bar{Y}}^{Y_{n}} \frac{\digamma_{4}(\bar{Y})}{\digamma_{4}(\varsigma)} d \varsigma\right)+\frac{\lambda e^{-\mu_{3} \tau_{3}}}{g} Z_{n}\right] \\
& +\frac{a}{\theta}\left(c+d \digamma_{4}(\bar{Y})\right) \digamma_{3}(\bar{H}) G\left(\frac{\digamma_{3}\left(H_{n}\right)}{\digamma_{3}(\bar{H})}\right) \\
& +\frac{m(1-\varepsilon) e^{-\mu_{1} \tau_{1}-\mu_{3} \tau_{3}}}{\alpha+m} \Lambda(\bar{F}, \bar{H}) \sum_{j=n-m_{1}}^{n-1} G\left(\frac{\Lambda\left(F_{j+1}, H_{j}\right)}{\Lambda(\bar{F}, \bar{H})}\right) \\
& +\varepsilon e^{-\mu_{2} \tau_{2}-\mu_{3} \tau_{3}} \Lambda(\bar{F}, \bar{H}) \sum_{j=n-m_{2}}^{n-1} G\left(\frac{\Lambda\left(F_{j+1}, H_{j}\right)}{\Lambda(\bar{F}, \bar{H})}\right) \\
& +a e^{-\mu_{3} \tau_{3}} \digamma_{2}(\bar{S}) \sum_{j=n-m_{3}}^{n-1} G\left(\frac{\digamma_{2}\left(S_{j+1}\right)}{\digamma_{2}(\bar{S})}\right) .
\end{aligned}
$$

Clearly, $\mathcal{W}_{n}\left(F_{n}, K_{n}, S_{n}, H_{n}, Y_{n}, Z_{n}\right)>0$ for all $F_{n}, K_{n}, S_{n}, H_{n}, Y_{n}, Z_{n}>0$ and $\mathcal{W}_{n}(\bar{F}, \bar{K}, \bar{S}$, $\bar{H}, \bar{Y}, 0)=0$. We compute $\Delta \mathcal{W}_{n}=\mathcal{W}_{n+1}-\mathcal{W}_{n}$ as follows:

$$
\begin{aligned}
\Delta \mathcal{W}_{n}= & \frac{1}{\phi(h)}\left[\gamma\left(F_{n+1}-\bar{F}-\int_{\bar{F}}^{F_{n+1}} \frac{\Lambda(\bar{F}, \bar{H})}{\Lambda(\zeta, \bar{H})} d \varsigma\right)\right. \\
& +\frac{m e^{-\mu_{3} \tau_{3}}}{\alpha+m}\left(K_{n+1}-\bar{K}-\int_{\bar{K}}^{K_{n+1}} \frac{\digamma_{1}(\bar{K})}{\digamma_{1}(\varsigma)} d \varsigma\right) \\
& +e^{-\mu_{3} \tau_{3}}\left(S_{n+1}-\bar{S}-\int_{\bar{S}}^{S_{n+1}} \frac{\digamma_{2}(\bar{S})}{\digamma_{2}(\varsigma)} d \varsigma\right)+\frac{a}{\theta}\left(H_{n+1}-\bar{H}-\int_{\bar{H}}^{H_{n+1}} \frac{\digamma_{3}(\bar{H})}{\digamma_{3}(\varsigma)} d \varsigma\right) \\
& \left.+\frac{a d}{\theta q}\left(Y_{n+1}-\bar{Y}-\int_{\bar{Y}}^{Y_{n+1}} \frac{\digamma_{4}(\bar{Y})}{\digamma_{4}(\varsigma)} d \varsigma\right)+\frac{\lambda e^{-\mu_{3} \tau_{3}}}{g} Z_{n+1}\right] \\
& +\frac{a}{\theta}\left(c+d \digamma_{4}(\bar{Y})\right) \digamma_{3}(\bar{H}) G\left(\frac{\digamma_{3}\left(H_{n+1}\right)}{\digamma_{3}(\bar{H})}\right) \\
& \left.+\frac{m(1-\varepsilon) e^{-\mu_{1} \tau_{1}-\mu_{3} \tau_{3}}}{\alpha+m} \bar{F}_{\bar{F}}, \bar{H}\right) \sum_{j=n-m_{1}+1}^{n} G\left(\frac{\Lambda\left(F_{j+1}, H_{j}\right)}{\Lambda(\bar{F}, \bar{H})}\right) \\
& +\varepsilon e^{-\mu_{2} \tau_{2}-\mu_{3} \tau_{3}} \Lambda(\bar{F}, \bar{H}) \sum_{j=n-m_{2}+1}^{n} G\left(\frac{\Lambda\left(F_{j+1}, H_{j}\right)}{\Lambda(\bar{F}, \bar{H})}\right) \\
& +a e^{-\mu_{3} \tau_{3}} \digamma_{2}(\bar{S}) \sum_{j=n-m_{3}+1}^{n} G\left(\frac{\digamma_{2}\left(S_{j+1}\right)}{\digamma_{2}(\bar{S})}\right)
\end{aligned}
$$




$$
\begin{aligned}
& -\frac{1}{\phi(h)}\left[\gamma\left(F_{n}-\bar{F}-\int_{\bar{F}}^{F_{n}} \frac{\Lambda(\bar{F}, \bar{H})}{\Lambda(\varsigma, \bar{H})} d \varsigma\right)+\frac{m e^{-\mu_{3} \tau_{3}}}{\alpha+m}\left(K_{n}-\bar{K}-\int_{\bar{K}}^{K_{n}} \frac{\digamma_{1}(\bar{K})}{\digamma_{1}(\varsigma)} d \varsigma\right)\right. \\
& +e^{-\mu_{3} \tau_{3}}\left(S_{n}-\bar{S}-\int_{\bar{S}}^{S_{n}} \frac{\digamma_{2}(\bar{S})}{\digamma_{2}(\varsigma)} d \varsigma\right)+\frac{a}{\theta}\left(H_{n}-\bar{H}-\int_{\bar{H}}^{H_{n}} \frac{\digamma_{3}(\bar{H})}{\digamma_{3}(\varsigma)} d \varsigma\right) \\
& \left.+\frac{a d}{\theta q}\left(Y_{n}-\bar{Y}-\int_{\bar{Y}}^{Y_{n}} \frac{\digamma_{4}(\bar{Y})}{\digamma_{4}(\varsigma)} d \varsigma\right)+\frac{\lambda e^{-\mu_{3} \tau_{3}}}{g} Z_{n}\right] \\
& -\frac{a}{\theta}\left(c+d \digamma_{4}(\bar{Y})\right) \digamma_{3}(\bar{H}) G\left(\frac{\digamma_{3}\left(H_{n}\right)}{\digamma_{3}(\bar{H})}\right) \\
& -\frac{m(1-\varepsilon) e^{-\mu_{1} \tau_{1}-\mu_{3} \tau_{3}}}{\alpha+m} \Lambda(\bar{F}, \bar{H}) \sum_{j=n-m_{1}}^{n-1} G\left(\frac{\Lambda\left(F_{j+1}, H_{j}\right)}{\Lambda(\bar{F}, \bar{H})}\right) \\
& -\varepsilon e^{-\mu_{2} \tau_{2}-\mu_{3} \tau_{3}} \Lambda(\bar{F}, \bar{H}) \sum_{j=n-m_{2}}^{n-1} G\left(\frac{\Lambda\left(F_{j+1}, H_{j}\right)}{\Lambda(\bar{F}, \bar{H})}\right) \\
& -a e^{-\mu_{3} \tau_{3}} \digamma_{2}(\bar{S}) \sum_{j=n-m_{3}}^{n-1} G\left(\frac{\digamma_{2}\left(S_{j+1}\right)}{\digamma_{2}(\bar{S})}\right), \\
& \Delta \mathcal{W}_{n}=\frac{1}{\phi(h)}\left[\gamma\left(F_{n+1}-F_{n}-\int_{F_{n}}^{F_{n+1}} \frac{\Lambda(\bar{F}, \bar{H})}{\Lambda(\varsigma, \bar{H})} d \varsigma\right)\right. \\
& +\frac{m e^{-\mu_{3} \tau_{3}}}{\alpha+m}\left(K_{n+1}-K_{n}-\int_{K_{n}}^{K_{n+1}} \frac{\digamma_{1}(\bar{K})}{\digamma_{1}(\varsigma)} d \varsigma\right) \\
& +e^{-\mu_{3} \tau_{3}}\left(S_{n+1}-S_{n}-\int_{S_{n}}^{S_{n+1}} \frac{\digamma_{2}(\bar{S})}{\digamma_{2}(\varsigma)} d \varsigma\right)+\frac{a}{\theta}\left(H_{n+1}-H_{n}-\int_{H_{n}}^{H_{n}+1} \frac{\digamma_{3}(\bar{H})}{\digamma_{3}(\varsigma)} d \varsigma\right) \\
& \left.+\frac{a d}{\theta q}\left(Y_{n+1}-Y_{n}-\int_{Y_{n}}^{Y_{n+1}} \frac{\digamma_{4}(\bar{Y})}{\digamma_{4}(\varsigma)} d \varsigma\right)+\frac{\lambda e^{-\mu_{3} \tau_{3}}}{g}\left(Z_{n+1}-Z_{n}\right)\right] \\
& +\frac{a}{\theta}\left(c+d \digamma_{4}(\bar{Y})\right) \digamma_{3}(\bar{H})\left[G\left(\frac{\digamma_{3}\left(H_{n+1}\right)}{\digamma_{3}(\bar{H})}\right)-G\left(\frac{\digamma_{3}\left(H_{n}\right)}{\digamma_{3}(\bar{H})}\right)\right] \\
& +\frac{m(1-\varepsilon) e^{-\mu_{1} \tau_{1}-\mu_{3} \tau_{3}}}{\alpha+m} \Lambda(\bar{F}, \bar{H})\left(\sum_{j=n-m_{1}+1}^{n} G\left(\frac{\Lambda\left(F_{j+1}, H_{j}\right)}{\Lambda(\bar{F}, \bar{H})}\right)\right. \\
& \left.-\sum_{j=n-m_{1}}^{n-1} G\left(\frac{\Lambda\left(F_{j+1}, H_{j}\right)}{\Lambda(\bar{F}, \bar{H})}\right)\right) \\
& +\varepsilon e^{-\mu_{2} \tau_{2}-\mu_{3} \tau_{3}} \Lambda(\bar{F}, \bar{H})\left(\sum_{j=n-m_{2}+1}^{n} G\left(\frac{\Lambda\left(F_{j+1}, H_{j}\right)}{\Lambda(\bar{F}, \bar{H})}\right)-\sum_{j=n-m_{2}}^{n-1} G\left(\frac{\Lambda\left(F_{j+1}, H_{j}\right)}{\Lambda(\bar{F}, \bar{H})}\right)\right) \\
& +a e^{-\mu_{3} \tau_{3}} \digamma_{2}(\bar{S})\left(\sum_{j=n-m_{3}+1}^{n} G\left(\frac{\digamma_{2}\left(S_{j+1}\right)}{\digamma_{2}(\bar{S})}\right)-\sum_{j=n-m_{3}}^{n-1} G\left(\frac{\digamma_{2}\left(S_{j+1}\right)}{\digamma_{2}(\bar{S})}\right)\right) .
\end{aligned}
$$

Using inequalities (45) and (46) by replacing $F^{*}, H^{*}, \rho^{*}$ with $\bar{F}, \bar{H}, \bar{\rho}$, we obtain

$$
\begin{aligned}
\Delta \mathcal{W}_{n} \leq & \frac{1}{\phi(h)}\left[\gamma\left(1-\frac{\Lambda(\bar{F}, \bar{H})}{\Lambda\left(F_{n+1}, \bar{H}\right)}\right)\left(F_{n+1}-F_{n}\right)+\frac{m e^{-\mu_{3} \tau_{3}}}{\alpha+m}\left(1-\frac{\digamma_{1}(\bar{K})}{\digamma_{1}\left(K_{n+1}\right)}\right)\left(K_{n+1}-K_{n}\right)\right. \\
& +e^{-\mu_{3} \tau_{3}}\left(1-\frac{\digamma_{2}(\bar{S})}{\digamma_{2}\left(S_{n+1}\right)}\right)\left(S_{n+1}-S_{n}\right)+\frac{a}{\theta}\left(1-\frac{\digamma_{3}(\bar{H})}{\digamma_{3}\left(H_{n+1}\right)}\right)\left(H_{n+1}-H_{n}\right)
\end{aligned}
$$




$$
\begin{aligned}
& \left.+\frac{a d}{\theta q}\left(1-\frac{\digamma_{4}(\bar{Y})}{\digamma_{4}\left(Y_{n+1}\right)}\right)\left(Y_{n+1}-Y_{n}\right)+\frac{\lambda e^{-\mu_{3} \tau_{3}}}{g}\left(Z_{n+1}-Z_{n}\right)\right] \\
& +\frac{a}{\theta}\left(c+d \digamma_{4}(\bar{Y})\right) \digamma_{3}(\bar{H})\left[\frac{\digamma_{3}\left(H_{n+1}\right)}{\digamma_{3}(\bar{H})}-\frac{\digamma_{3}\left(H_{n}\right)}{\digamma_{3}(\bar{H})}+\ln \left(\frac{\digamma_{3}\left(H_{n}\right)}{\digamma_{3}\left(H_{n+1}\right)}\right)\right] \\
& +\frac{m(1-\varepsilon) e^{-\mu_{1} \tau_{1}-\mu_{3} \tau_{3}}}{\alpha+m} \Lambda(\bar{F}, \bar{H})\left(\frac{\Lambda\left(F_{n+1}, H_{n}\right)}{\Lambda(\bar{F}, \bar{H})}-\frac{\Lambda\left(F_{n-m_{1}+1}, H_{n-m_{1}}\right)}{\Lambda(\bar{F}, \bar{H})}\right. \\
& \left.+\ln \left(\frac{\Lambda\left(F_{n-m_{1}+1}, H_{n-m_{1}}\right)}{\Lambda\left(F_{n+1}, H_{n}\right)}\right)\right) \\
& +\varepsilon e^{-\mu_{2} \tau_{2}-\mu_{3} \tau_{3}} \Lambda(\bar{F}, \bar{H})\left(\frac{\Lambda\left(F_{n+1}, H_{n}\right)}{\Lambda(\bar{F}, \bar{H})}-\frac{\Lambda\left(F_{n-m_{2}+1}, H_{n-m_{2}}\right)}{\Lambda(\bar{F}, \bar{H})}\right. \\
& \left.+\ln \left(\frac{\Lambda\left(F_{n-m_{2}+1}, H_{n-m_{2}}\right)}{\Lambda\left(F_{n+1}, H_{n}\right)}\right)\right) \\
& +a e^{-\mu_{3} \tau_{3}} \digamma_{2}(\bar{S})\left(\frac{\digamma_{2}\left(S_{n+1}\right)}{\digamma_{2}(\bar{S})}-\frac{\digamma_{2}\left(S_{n-m_{3}+1}\right)}{\digamma_{2}(\bar{S})}+\ln \left(\frac{\digamma_{2}\left(S_{n-m_{3}+1}\right)}{\digamma_{2}\left(S_{n+1}\right)}\right)\right) .
\end{aligned}
$$

From Eqs. (11)-(16) we have

$$
\begin{aligned}
\Delta \mathcal{W}_{n} \leq & \gamma\left(1-\frac{\Lambda(\bar{F}, \bar{H})}{\Lambda\left(F_{n+1}, \bar{H}\right)}\right)\left[\Theta\left(F_{n+1}\right)-\Lambda\left(F_{n+1}, H_{n}\right)\right] \\
& +\frac{m e^{-\mu_{3} \tau_{3}}}{\alpha+m}\left(1-\frac{\digamma_{1}(\bar{K})}{\digamma_{1}\left(K_{n+1}\right)}\right)\left[(1-\varepsilon) e^{-\mu_{1} \tau_{1}} \Lambda\left(F_{n-m_{1}+1}, H_{n-m_{1}}\right)\right. \\
& \left.-(\alpha+m) \digamma_{1}\left(K_{n+1}\right)\right] \\
& +e^{-\mu_{3} \tau_{3}}\left(1-\frac{\digamma_{2}(\bar{S})}{\digamma_{2}\left(S_{n+1}\right)}\right)\left[\varepsilon e^{-\mu_{2} \tau_{2}} \Lambda\left(F_{n-m_{2}+1}, H_{n-m_{2}}\right)+m \digamma_{1}\left(K_{n+1}\right)-a \digamma_{2}\left(S_{n+1}\right)\right. \\
& \left.-\lambda \digamma_{2}\left(S_{n+1}\right) \digamma_{5}\left(Z_{n+1}\right)\right] \\
& +\frac{a}{\theta}\left(1-\frac{\digamma_{3}(\bar{H})}{\digamma_{3}\left(H_{n+1}\right)}\right)\left[\theta e^{-\mu_{3} \tau_{3}} \digamma_{2}\left(S_{n-m_{3}+1}\right)-c \digamma_{3}\left(H_{n+1}\right)-d \digamma_{3}\left(H_{n+1}\right) \digamma_{4}\left(Y_{n+1}\right)\right] \\
& +\frac{a d}{\theta q}\left(1-\frac{\digamma_{4}(\bar{Y})}{\digamma_{4}\left(Y_{n+1}\right)}\right)\left[q \digamma_{3}\left(H_{n+1}\right) \digamma_{4}\left(Y_{n+1}\right)-\eta \digamma_{4}\left(Y_{n+1}\right)\right] \\
& +\frac{\lambda e^{-\mu_{3} \tau_{3}}}{g}\left[g \digamma_{2}\left(S_{n+1}\right) \digamma_{5}\left(Z_{n+1}\right)-\xi \digamma_{5}\left(Z_{n+1}\right)\right] \\
& +\frac{a}{\theta}\left(c+d \digamma_{4}(\bar{Y})\right) \digamma_{3}(\bar{H})\left[\frac{\digamma_{3}\left(H_{n+1}\right)}{\digamma_{3}(\bar{H})}-\frac{\digamma_{3}\left(H_{n}\right)}{\digamma_{3}(\bar{H})}+\ln \left(\frac{\digamma_{3}\left(H_{n}\right)}{\digamma_{3}\left(H_{n+1}\right)}\right)\right] \\
& +\frac{m(1-\varepsilon) e^{-\mu_{1} \tau_{1}-\mu_{3} \tau_{3}}}{\alpha+m} \Lambda(\bar{F}, \bar{H})\left(\frac{\Lambda\left(F_{n+1}, H_{n}\right)}{\Lambda(\bar{F}, \bar{H})}-\frac{\Lambda\left(F_{n-m_{1}+1}, H_{n-m_{1}}\right)}{\Lambda(\bar{F}, \bar{H})}\right. \\
& +a e^{-\mu_{3} \tau_{3}} \digamma_{2}(\bar{S})\left(\frac{\digamma_{2}\left(S_{n+1}\right)}{\digamma_{2}(\bar{S})}-\frac{\digamma_{2}\left(S_{n-m_{3}+1}\right)}{\digamma_{2}(\bar{S})}+\ln \left(\frac{\digamma_{2}\left(S_{n-m_{3}+1}\right)}{\digamma_{2}\left(S_{n+1}\right)}\right)\right) \\
& \left.+\ln \left(\frac{\Lambda\left(F_{n-m_{1}+1}, H_{n-m_{1}}\right)}{\Lambda\left(F_{n+1}, H_{n}\right)}\right)\right) \\
& +\varepsilon e^{-\mu_{2} \tau_{2}-\mu_{3} \tau_{3}} \Lambda(\bar{F}, \bar{H})\left(\frac{\Lambda\left(F_{n+1}, H_{n}\right)}{\Lambda(\bar{F}, \bar{H})}-\frac{\Lambda\left(F_{n-m_{2}+1}, H_{n-m_{2}}\right)}{\Lambda(\bar{F}, \bar{H})}\right. \\
+ & \left.\ln \left(\frac{\Lambda\left(F_{n-m_{2}+1}, H_{n-m_{2}}\right)}{\Lambda\left(F_{n+1}, H_{n}\right)}\right)\right) \\
&
\end{aligned}
$$




$$
\begin{aligned}
= & \gamma\left(1-\frac{\Lambda(\bar{F}, \bar{H})}{\Lambda\left(F_{n+1}, \bar{H}\right)}\right)\left(\Theta\left(F_{n+1}\right)-\Theta(\bar{F})\right)+\gamma \Theta(\bar{F})\left(1-\frac{\Lambda(\bar{F}, \bar{H})}{\Lambda\left(F_{n+1}, \bar{H}\right)}\right) \\
& +\gamma \frac{\Lambda(\bar{F}, \bar{H})}{\Lambda\left(F_{n+1}, \bar{H}\right)} \Lambda\left(\digamma_{n+1}, H_{n}\right)-\frac{m(1-\varepsilon) e^{-\mu_{1} \tau_{1}-\mu_{3} \tau_{3}}}{\alpha+m} \Lambda\left(\digamma_{n-m_{1}+1}, H_{n-m_{1}}\right) \frac{\digamma_{1}(\bar{K})}{\digamma_{1}\left(K_{n+1}\right)} \\
& +m e^{-\mu_{3} \tau_{3}} \digamma_{1}(\bar{K})-\varepsilon e^{-\mu_{2} \tau_{2}-\mu_{3} \tau_{3}} \Lambda\left(F_{n-m_{2}+1}, H_{n-m_{2}}\right) \frac{\digamma_{2}(\bar{S})}{\digamma_{2}\left(S_{n+1}\right)} \\
& -m e^{-\mu_{3} \tau_{3}} \digamma_{1}\left(K_{n+1}\right) \frac{\digamma_{2}(\bar{S})}{\digamma_{2}\left(S_{n+1}\right)} \\
& +a e^{-\mu_{3} \tau_{3}} \digamma_{2}(\bar{S})+\lambda e^{-\mu_{3} \tau_{3}} \digamma_{2}(\bar{S}) \digamma_{5}\left(Z_{n+1}\right)-a e^{-\mu_{3} \tau_{3}} \digamma_{2}\left(S_{n-m_{3}+1}\right) \frac{\digamma_{3}(\bar{H})}{\digamma_{3}\left(H_{n+1}\right)} \\
& +\frac{a c}{\theta} \digamma_{3}(\bar{H}) \\
& +\frac{a d}{\theta} \digamma_{3}(\bar{H}) \digamma_{4}\left(Y_{n+1}\right)-\frac{a d \eta}{\theta q} \digamma_{4}\left(Y_{n+1}\right)+\frac{a d \eta}{\theta q} \digamma_{4}(\bar{Y})-\frac{\lambda e^{-\mu_{3} \tau_{3} \xi}}{g} \digamma_{5}\left(Z_{n+1}\right) \\
& -\frac{a}{\theta}\left(c+d \digamma_{4}(\bar{Y})\right) \digamma_{3}\left(H_{n}\right)+\frac{a}{\theta}\left(c+d \digamma_{4}(\bar{Y})\right) \digamma_{3}(\bar{H}) \ln \left(\frac{\digamma_{3}\left(H_{n}\right)}{\digamma_{3}\left(H_{n+1}\right)}\right) \\
& +\frac{m(1-\varepsilon) e^{-\mu_{1} \tau_{1}-\mu_{3} \tau_{3}}}{\alpha+m} \Lambda(\bar{F}, \bar{H}) \ln \left(\frac{\Lambda\left(F_{n-m_{1}+1}, H_{n-m_{1}}\right)}{\Lambda\left(F_{n+1}, H_{n}\right)}\right) \\
& +\varepsilon e^{-\mu_{2} \tau_{2}-\mu_{3} \tau_{3}} \Lambda(\bar{F}, \bar{H}) \ln \left(\frac{\Lambda\left(F_{n-m_{2}+1}, H_{n-m_{2}}\right)}{\Lambda\left(F_{n+1}, H_{n}\right)}\right) \\
& +a e^{-\mu_{3} \tau_{3}} \digamma_{2}(\bar{S}) \ln \left(\frac{\digamma_{2}\left(S_{n-m_{3}+1}\right)}{\digamma_{2}\left(S_{n+1}\right)}\right) .
\end{aligned}
$$

Using the conditions of $\bar{Q}$

$$
\begin{aligned}
& \Theta(\bar{F})=\Lambda(\bar{F}, \bar{H}), \\
& (1-\varepsilon) e^{-\mu_{1} \tau_{1}} \Lambda(\bar{F}, \bar{H})=(\alpha+m) \digamma_{1}(\bar{K}), \\
& \varepsilon e^{-\mu_{2} \tau_{2}-\mu_{3} \tau_{3}} \Lambda(\bar{F}, \bar{H})+m e^{-\mu_{3} \tau_{3}} \digamma_{1}(\bar{K})=\gamma \Lambda(\bar{F}, \bar{H})=a e^{-\mu_{3} \tau_{3}} \digamma_{2}(\bar{S}), \\
& \theta e^{-\mu_{3} \tau_{3}} \digamma_{2}(\bar{S})=\left(c+d \digamma_{4}(\bar{Y})\right) \digamma_{3}(\bar{H}), \\
& \eta=q \digamma_{3}(\bar{H}),
\end{aligned}
$$

we get

$$
\begin{aligned}
\Delta \mathcal{W}_{n} \leq & \gamma\left(1-\frac{\Lambda(\bar{F}, \bar{H})}{\Lambda\left(F_{n+1}, \bar{H}\right)}\right)\left(\Theta\left(F_{n+1}\right)-\Theta(\bar{F})\right)+\gamma \Lambda(\bar{F}, \bar{H})\left(1-\frac{\Lambda(\bar{F}, \bar{H})}{\Lambda\left(F_{n+1}, \bar{H}\right)}\right) \\
& +\gamma \Lambda(\bar{F}, \bar{H}) \frac{\Lambda\left(F_{n+1}, H_{n}\right)}{\Lambda\left(F_{n+1}, \bar{H}\right)} \\
& -\frac{m(1-\varepsilon) e^{-\mu_{1} \tau_{1}-\mu_{3} \tau_{3}}}{\alpha+m} \Lambda(\bar{F}, \bar{H}) \frac{\Lambda\left(F_{n-m_{1}+1}, H_{n-m_{1}}\right) \digamma_{1}(\bar{K})}{\Lambda(\bar{F}, \bar{H}) \digamma_{1}\left(K_{n+1}\right)} \\
& +\frac{m(1-\varepsilon) e^{-\mu_{1} \tau_{1}-\mu_{3} \tau_{3}}}{\alpha+m} \Lambda(\bar{F}, \bar{H}) \\
& -\varepsilon e^{-\mu_{2} \tau_{2}-\mu_{3} \tau_{3}} \Lambda(\bar{F}, \bar{H}) \frac{\Lambda\left(F_{n-m_{2}+1}, H_{n-m_{2}}\right) \digamma_{2}(\bar{S})}{\Lambda(\bar{F}, \bar{H}) \digamma_{2}\left(S_{n+1}\right)}
\end{aligned}
$$




$$
\begin{aligned}
& -\frac{m(1-\varepsilon) e^{-\mu_{1} \tau_{1}-\mu_{3} \tau_{3}}}{\alpha+m} \Lambda(\bar{F}, \bar{H}) \frac{\digamma_{1}\left(K_{n+1}\right) \digamma_{2}(\bar{S})}{\digamma_{1}(\bar{K}) \digamma_{2}\left(S_{n+1}\right)}+\gamma \Lambda(\bar{F}, \bar{H}) \\
& -\gamma \Lambda(\bar{F}, \bar{H}) \frac{\digamma_{3}(\bar{H}) \digamma_{2}\left(S_{n-m_{3}+1}\right)}{\digamma_{3}\left(H_{n+1}\right) \digamma_{2}(\bar{S})} \\
& +\gamma \Lambda(\bar{F}, \bar{H})-\gamma \Lambda(\bar{F}, \bar{H}) \frac{\digamma_{3}\left(H_{n}\right)}{\digamma_{3}(\bar{H})}+\lambda e^{-\mu_{3} \tau_{3}}\left(\digamma_{2}(\bar{S})-\frac{\xi}{g}\right) \digamma_{5}\left(Z_{n+1}\right) \\
& +\gamma \Lambda(\bar{F}, \bar{H}) \ln \left(\frac{\digamma_{3}\left(H_{n}\right)}{\digamma_{3}\left(H_{n+1}\right)}\right) \\
& +\frac{m(1-\varepsilon) e^{-\mu_{1} \tau_{1}-\mu_{3} \tau_{3}}}{\alpha+m} \Lambda(\bar{F}, \bar{H}) \ln \left(\frac{\Lambda\left(F_{n-m_{1}+1}, H_{n-m_{1}}\right)}{\Lambda\left(F_{n+1}, H_{n}\right)}\right) \\
& +\varepsilon e^{-\mu_{2} \tau_{2}-\mu_{3} \tau_{3}} \Lambda(\bar{F}, \bar{H}) \ln \left(\frac{\Lambda\left(F_{n-m_{2}+1}, H_{n-m_{2}}\right)}{\Lambda\left(F_{n+1}, H_{n}\right)}\right) \\
& +\gamma \Lambda(\bar{F}, \bar{H}) \ln \left(\frac{\digamma_{2}\left(S_{n-m_{3}+1}\right)}{\digamma_{2}\left(S_{n+1}\right)}\right) \\
& =\gamma\left(1-\frac{\Lambda(\bar{F}, \bar{H})}{\Lambda\left(F_{n+1}, \bar{H}\right)}\right)\left(\Theta\left(F_{n+1}\right)-\Theta(\bar{F})\right) \\
& +\frac{m(1-\varepsilon) e^{-\mu_{1} \tau_{1}-\mu_{3} \tau_{3}}}{\alpha+m} \Lambda(\bar{F}, \bar{H})\left[5-\frac{\Lambda(\bar{F}, \bar{H})}{\Lambda\left(F_{n+1}, \bar{H}\right)}\right. \\
& -\frac{\Lambda\left(F_{n-m_{1}+1}, H_{n-m_{1}}\right) \digamma_{1}(\bar{K})}{\Lambda(\bar{F}, \bar{H}) \digamma_{1}\left(K_{n+1}\right)} \\
& -\frac{\digamma_{1}\left(K_{n+1}\right) \digamma_{2}(\bar{S})}{\digamma_{1}(\bar{K}) \digamma_{2}\left(S_{n+1}\right)}-\frac{\digamma_{3}(\bar{H}) \digamma_{2}\left(S_{n-m_{3}+1}\right)}{\digamma_{3}\left(H_{n+1}\right) \digamma_{2}(\bar{S})}-\frac{\Lambda\left(F_{n+1}, \bar{H}\right) \digamma_{3}\left(H_{n}\right)}{\Lambda\left(F_{n+1}, H_{n}\right) \digamma_{3}(\bar{H})} \\
& \left.+\ln \left(\frac{\Lambda\left(F_{n-m_{1}+1}, H_{n-m_{1}}\right) \digamma_{2}\left(S_{n-m_{3}+1}\right) \digamma_{3}\left(H_{n}\right)}{\Lambda\left(F_{n+1}, H_{n}\right) \digamma_{2}\left(S_{n+1}\right) \digamma_{3}\left(H_{n+1}\right)}\right)\right] \\
& +\varepsilon e^{-\mu_{2} \tau_{2}-\mu_{3} \tau_{3}} \Lambda(\bar{F}, \bar{H})\left[4-\frac{\Lambda(\bar{F}, \bar{H})}{\Lambda\left(F_{n+1}, \bar{H}\right)}\right. \\
& -\frac{\Lambda\left(\digamma_{n-m_{2}+1}, H_{n-m_{2}}\right) \digamma_{2}(\bar{S})}{\Lambda(\bar{F}, \bar{H}) \digamma_{2}\left(S_{n+1}\right)}-\frac{\digamma_{3}(\bar{H}) \digamma_{2}\left(S_{n-m_{3}+1}\right)}{\digamma_{3}\left(H_{n+1}\right) \digamma_{2}(\bar{S})} \\
& \left.-\frac{\Lambda\left(F_{n+1}, \bar{H}\right) \digamma_{3}\left(H_{n}\right)}{\Lambda\left(F_{n+1}, H_{n}\right) \digamma_{3}(\bar{H})}+\ln \left(\frac{\Lambda\left(F_{n-m_{2}+1}, H_{n-m_{2}}\right) \digamma_{2}\left(S_{n-m_{3}+1}\right) \digamma_{3}\left(H_{n}\right)}{\Lambda\left(F_{n+1}, H_{n}\right) \digamma_{2}\left(S_{n+1}\right) \digamma_{3}\left(H_{n+1}\right)}\right)\right] \\
& +\lambda e^{-\mu_{3} \tau_{3}}\left(\digamma_{2}(\bar{S})-\digamma_{2}(\widetilde{S})\right) \digamma_{5}\left(Z_{n+1}\right) \\
& +\gamma \Lambda(\bar{F}, \bar{H})\left[-1+\frac{\Lambda\left(F_{n+1}, \bar{H}\right) \digamma_{3}\left(H_{n}\right)}{\Lambda\left(F_{n+1}, H_{n}\right) \digamma_{3}(\bar{H})}+\frac{\Lambda\left(F_{n+1}, H_{n}\right)}{\Lambda\left(F_{n+1}, \bar{H}\right)}-\frac{\digamma_{3}\left(H_{n}\right)}{\digamma_{3}(\bar{H})}\right], \\
& \Delta \mathcal{W}_{n} \leq \gamma\left(1-\frac{\Lambda(\bar{F}, \bar{H})}{\Lambda\left(F_{n+1}, \bar{H}\right)}\right)\left(\Theta\left(F_{n+1}\right)-\Theta(\bar{F})\right) \\
& -\frac{m(1-\varepsilon) e^{-\mu_{1} \tau_{1}-\mu_{3} \tau_{3}}}{\alpha+m} \Lambda(\bar{F}, \bar{H})\left[G\left(\frac{\Lambda(\bar{F}, \bar{H})}{\Lambda\left(F_{n+1}, \bar{H}\right)}\right)\right. \\
& +G\left(\frac{\Lambda\left(F_{n-m_{1}+1}, H_{n-m_{1}}\right) \digamma_{1}(\bar{K})}{\Lambda(\bar{F}, \bar{H}) \digamma_{1}\left(K_{n+1}\right)}\right) \\
& +G\left(\frac{\digamma_{1}\left(K_{n+1}\right) \digamma_{2}(\bar{S})}{\digamma_{1}(\bar{K}) \digamma_{2}\left(S_{n+1}\right)}\right)+G\left(\frac{\digamma_{3}(\bar{H}) \digamma_{2}\left(S_{n-m_{3}+1}\right)}{\digamma_{3}\left(H_{n+1}\right) \digamma_{2}(\bar{S})}\right)
\end{aligned}
$$




$$
\begin{aligned}
& \left.+G\left(\frac{\Lambda\left(F_{n+1}, \bar{H}\right) \digamma_{3}\left(H_{n}\right)}{\Lambda\left(F_{n+1}, H_{n}\right) \digamma_{3}(\bar{H})}\right)\right]-\varepsilon e^{-\mu_{2} \tau_{2}-\mu_{3} \tau_{3}} \Lambda(\bar{F}, \bar{H})\left[G\left(\frac{\Lambda(\bar{F}, \bar{H})}{\Lambda\left(F_{n+1}, \bar{H}\right)}\right)\right. \\
& +G\left(\frac{\Lambda\left(F_{n-m_{2}+1}, H_{n-m_{2}}\right) \digamma_{2}(\bar{S})}{\Lambda(\bar{F}, \bar{H}) \digamma_{2}\left(S_{n+1}\right)}\right)+G\left(\frac{\digamma_{3}(\bar{H}) \digamma_{2}\left(S_{n-m_{3}+1}\right)}{\digamma_{3}\left(H_{n+1}\right) \digamma_{2}(\bar{S})}\right) \\
& \left.+G\left(\frac{\Lambda\left(F_{n+1}, \bar{H}\right) \digamma_{3}\left(H_{n}\right)}{\Lambda\left(F_{n+1}, H_{n}\right) \digamma_{3}(\bar{H})}\right)\right] \\
& +\lambda e^{-\mu_{3} \tau_{3}} \digamma_{2}(\widetilde{S})\left(\mathcal{R}_{2}^{Z}-1\right) \digamma_{5}\left(Z_{n+1}\right) \\
& +\gamma \Lambda(\bar{F}, \bar{H})\left(1-\frac{\Lambda\left(F_{n+1}, \bar{H}\right)}{\Lambda\left(F_{n+1}, H_{n}\right)}\right)\left(\frac{\Lambda\left(F_{n+1}, H_{n}\right)}{\Lambda\left(F_{n+1}, \bar{H}\right)}-\frac{\digamma_{3}\left(H_{n}\right)}{\digamma_{3}(\bar{H})}\right) .
\end{aligned}
$$

Using Conditions $\mathrm{C} 1-\mathrm{C} 4$, we get that the first and last terms of Eq. (52) are less than or equal to zero. Moreover, if $\mathcal{R}_{2}^{Z} \leq 1$, we get $\Delta \mathcal{W}_{n} \leq 0$, and thus $\mathcal{W}_{n}$ is a monotone

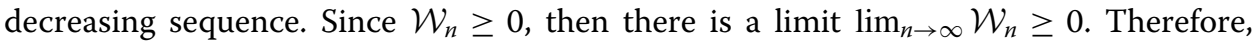
$\lim _{n \rightarrow \infty} \Delta \mathcal{W}_{n}=0$, which implies that $\lim _{n \rightarrow \infty} F_{n}=\bar{F}, \lim _{n \rightarrow \infty} K_{n}=\bar{K}, \lim _{n \rightarrow \infty} S_{n}=\bar{S}$, $\lim _{n \rightarrow \infty} H_{n}=\bar{H}$, and $\lim _{n \rightarrow \infty}\left(\mathcal{R}_{2}^{Z}-1\right) \digamma_{5}\left(Z_{n+1}\right)=0$ We have two cases:

- $\mathcal{R}_{2}^{Z}=1$, then from Eq. (13)

$$
0=\varepsilon e^{-\mu_{2} \tau_{2}} \Lambda(\overline{F, H})+m \digamma_{1}(\bar{K})-a \digamma_{2}(\bar{S})-\lambda \digamma_{2}(\bar{S}) \lim _{n \rightarrow \infty} \digamma_{5}\left(Z_{n+1}\right),
$$

we get $\lim _{n \rightarrow \infty} Z_{n}=0$. From Eq. (14) we get

$$
0=\theta e^{-\mu_{3} \tau_{3}} \digamma_{2}(\bar{S})-c \digamma_{3}(\bar{H})-d \digamma_{3}(\bar{H}) \lim _{n \rightarrow \infty} \digamma_{4}\left(Y_{n+1}\right) .
$$

This gives $\lim _{n \rightarrow \infty} Y_{n}=\bar{Y}$.

- $\mathcal{R}_{2}^{Z}<1, \lim _{n \rightarrow \infty} \digamma_{5}\left(Z_{n}\right)=0$. From Eq. (54) we get $\lim _{n \rightarrow \infty} Y_{n}=\bar{Y}$. Hence, $\bar{Q}$ is G.A.S.

Proof of Theorem 4 Define $\mathcal{M}_{n}\left(F_{n}, K_{n}, S_{n}, H_{n}, Y_{n}, Z_{n}\right)$ :

$$
\begin{aligned}
\mathcal{M}_{n}= & \frac{1}{\phi(h)}\left[\gamma\left(F_{n}-\widehat{F}-\int_{\widehat{F}}^{F_{n}} \frac{\Lambda(\widehat{F}, \widehat{H})}{\Lambda(\varsigma, \widehat{H})} d \varsigma\right)+\frac{m e^{-\mu_{3} \tau_{3}}}{\alpha+m}\left(K_{n}-\widehat{K}-\int_{\widehat{K}}^{K_{n}} \frac{\digamma_{1}(\widehat{K})}{\digamma_{1}(\varsigma)} d \varsigma\right)\right. \\
& +e^{-\mu_{3} \tau_{3}}\left(S_{n}-\widehat{S}-\int_{\widehat{S}}^{S_{n}} \frac{\digamma_{2}(\widehat{S})}{\digamma_{2}(\varsigma)} d \varsigma\right)+\frac{\left(a+\lambda \digamma_{5}(\widehat{Z})\right)}{\theta}\left(H_{n}-\widehat{H}-\int_{\widehat{H}}^{H_{n}} \frac{\digamma_{3}(\widehat{H})}{\digamma_{3}(\varsigma)} d \varsigma\right) \\
& \left.+\frac{d\left(a+\lambda \digamma_{5}(\widehat{Z})\right)}{q \theta} Y_{n}+\frac{\lambda e^{-\mu_{3} \tau_{3}}}{g}\left(Z_{n}-\widehat{Z}-\int_{\widehat{Z}}^{Z_{n}} \frac{\digamma_{5}(\widehat{Z})}{\digamma_{5}(\varsigma)} d \varsigma\right)\right] \\
& +\frac{c}{\theta}\left(a+\lambda \digamma_{5}(\widehat{Z})\right) \digamma_{3}(\widehat{H}) G\left(\frac{\digamma_{3}\left(H_{n}\right)}{\digamma_{3}(\widehat{H})}\right) \\
& +\frac{m(1-\varepsilon) e^{-\mu_{1} \tau_{1}-\mu_{3} \tau_{3}}}{\alpha+m} \Lambda(\widehat{F}, \widehat{H}) \sum_{j=n-m_{1}}^{n-1} G\left(\frac{\Lambda\left(F_{j+1}, H_{j}\right)}{\Lambda(\widehat{F}, \widehat{H})}\right) \\
& +\varepsilon e^{-\mu_{2} \tau_{2}-\mu_{3} \tau_{3}} \Lambda(\widehat{F}, \widehat{H}) \sum_{j=n-m_{2}}^{n-1} G\left(\frac{\Lambda\left(F_{j+1}, H_{j}\right)}{\Lambda(\widehat{F}, \widehat{H})}\right)+\gamma \Lambda(\widehat{F}, \widehat{H}) \sum_{j=n-m_{3}}^{n-1} G\left(\frac{\digamma_{2}\left(S_{j+1}\right)}{\digamma_{2}(\widehat{S})}\right) .
\end{aligned}
$$


Clearly, $\mathcal{M}_{n}\left(F_{n}, K_{n}, S_{n}, H_{n}, Y_{n}, Z_{n}\right)>0$ for all $F_{n}, K_{n}, S_{n}, H_{n}, Y_{n}, Z_{n}>0$ and $\mathcal{M}_{n}(\widehat{F}, \widehat{K}, \widehat{S}, \widehat{H}$, $0, \widehat{Z})=0$. We compute $\Delta \mathcal{M}_{n}=\mathcal{M}_{n}-\mathcal{M}_{n}$ as follows:

$$
\begin{aligned}
& \Delta \mathcal{M}_{n}=\frac{1}{\phi(h)}\left[\gamma\left(F_{n+1}-\widehat{F}-\int_{\widehat{F}}^{F_{n+1}} \frac{\Lambda(\widehat{F}, \widehat{H})}{\Lambda(\varsigma, \widehat{H})} d \varsigma\right)\right. \\
& +\frac{m e^{-\mu_{3} \tau_{3}}}{\alpha+m}\left(K_{n+1}-\widehat{K}-\int_{\widehat{K}}^{K_{n+1}} \frac{\digamma_{1}(\widehat{K})}{\digamma_{1}(\varsigma)} d \varsigma\right) \\
& +e^{-\mu_{3} \tau_{3}}\left(S_{n+1}-\widehat{S}-\int_{\widehat{S}}^{S_{n+1}} \frac{\digamma_{2}(\widehat{S})}{\digamma_{2}(\varsigma)} d \varsigma\right) \\
& +\frac{\left(a+\lambda \digamma_{5}(\widehat{Z})\right)}{\theta}\left(H_{n+1}-\widehat{H}-\int_{\widehat{H}}^{H_{n+1}} \frac{\digamma_{3}(\widehat{H})}{\digamma_{3}(\varsigma)} d \varsigma\right) \\
& \left.+\frac{d\left(a+\lambda \digamma_{5}(\widehat{Z})\right)}{q \theta} Y_{n+1}+\frac{\lambda e^{-\mu_{3} \tau_{3}}}{g}\left(Z_{n+1}-\widehat{Z}-\int_{\widehat{Z}}^{Z_{n+1}} \frac{\digamma_{5}(\widehat{Z})}{\digamma_{5}(\varsigma)} d \varsigma\right)\right] \\
& +\frac{c}{\theta}\left(a+\lambda \digamma_{5}(\widehat{Z})\right) \digamma_{3}(\widehat{H}) G\left(\frac{\digamma_{3}\left(H_{n+1}\right)}{\digamma_{3}(\widehat{H})}\right) \\
& +\frac{m(1-\varepsilon) e^{-\mu_{1} \tau_{1}-\mu_{3} \tau_{3}}}{\alpha+m} \Lambda(\widehat{F}, \widehat{H}) \sum_{j=n-m_{1}+1}^{n} G\left(\frac{\Lambda\left(F_{j+1}, H_{j}\right)}{\Lambda(\widehat{F}, \widehat{H})}\right) \\
& +\varepsilon e^{-\mu_{2} \tau_{2}-\mu_{3} \tau_{3}} \Lambda(\widehat{F}, \widehat{H}) \sum_{j=n-m_{2}+1}^{n} G\left(\frac{\Lambda\left(F_{j+1}, H_{j}\right)}{\Lambda(\widehat{F}, \widehat{H})}\right) \\
& +\gamma \Lambda(\widehat{F}, \widehat{H}) \sum_{j=n-m_{3}+1}^{n} G\left(\frac{\digamma_{2}\left(S_{j+1}\right)}{\digamma_{2}(\widehat{S})}\right) \\
& -\frac{1}{\phi(h)}\left[\gamma\left(F_{n}-\widehat{F}-\int_{\widehat{F}}^{F_{n}} \frac{\Lambda(\widehat{F}, \widehat{H})}{\Lambda(\varsigma, \widehat{H})} d \varsigma\right)\right. \\
& +\frac{m e^{-\mu_{3} \tau_{3}}}{\alpha+m}\left(K_{n}-\widehat{K}-\int_{\widehat{K}}^{K_{n}} \frac{\digamma_{1}(\widehat{K})}{\digamma_{1}(\varsigma)} d \varsigma\right) \\
& +e^{-\mu_{3} \tau_{3}}\left(S_{n}-\widehat{S}-\int_{\widehat{S}}^{S_{n}} \frac{\digamma_{2}(\widehat{S})}{\digamma_{2}(\varsigma)} d \varsigma\right) \\
& +\frac{\left(a+\lambda \digamma_{5}(\widehat{Z})\right)}{\theta}\left(H_{n}-\widehat{H}-\int_{\widehat{H}}^{H_{n}} \frac{\digamma_{3}(\widehat{H})}{\digamma_{3}(\varsigma)} d \varsigma\right) \\
& \left.+\frac{d\left(a+\lambda \digamma_{5}(\widehat{Z})\right)}{q \theta} Y_{n}+\frac{\lambda e^{-\mu_{3} \tau_{3}}}{g}\left(Z_{n}-\widehat{Z}-\int_{\widehat{Z}}^{Z_{n}} \frac{\digamma_{5}(\widehat{Z})}{\digamma_{5}(\varsigma)} d \varsigma\right)\right] \\
& -\frac{c}{\theta}\left(a+\lambda \digamma_{5}(\widehat{Z})\right) \digamma_{3}(\widehat{H}) G\left(\frac{\digamma_{3}\left(H_{n}\right)}{\digamma_{3}(\widehat{H})}\right) \\
& -\frac{m(1-\varepsilon) e^{-\mu_{1} \tau_{1}-\mu_{3} \tau_{3}}}{\alpha+m} \Lambda(\widehat{F}, \widehat{H}) \sum_{j=n-m_{1}}^{n-1} G\left(\frac{\Lambda\left(F_{j+1}, H_{j}\right)}{\Lambda(\widehat{F}, \widehat{H})}\right) \\
& -\varepsilon e^{-\mu_{2} \tau_{2}-\mu_{3} \tau_{3}} \Lambda(\widehat{F}, \widehat{H}) \sum_{j=n-m_{2}}^{n-1} G\left(\frac{\Lambda\left(F_{j+1}, H_{j}\right)}{\Lambda(\widehat{F}, \widehat{H})}\right) \\
& -\gamma \Lambda(\widehat{F}, \widehat{H}) \sum_{j=n-m_{3}}^{n-1} G\left(\frac{\digamma_{2}\left(S_{j+1}\right)}{\digamma_{2}(\widehat{S})}\right)
\end{aligned}
$$




$$
\begin{aligned}
& \Delta \mathcal{M}_{n}=\frac{1}{\phi(h)}\left[\gamma\left(F_{n+1}-F_{n}-\int_{F_{n}}^{F_{n+1}} \frac{\Lambda(\widehat{F}, \widehat{H})}{\Lambda(\varsigma, \widehat{H})} d \varsigma\right)\right. \\
& +\frac{m e^{-\mu_{3} \tau_{3}}}{\alpha+m}\left(K_{n+1}-K_{n}-\int_{K_{n}}^{K_{n+1}} \frac{\digamma_{1}(\widehat{K})}{\digamma_{1}(\varsigma)} d \varsigma\right) \\
& +e^{-\mu_{3} \tau_{3}}\left(S_{n+1}-S_{n}-\int_{S_{n}}^{S_{n+1}} \frac{\digamma_{2}(\widehat{S})}{\digamma_{2}(\varsigma)} d \varsigma\right) \\
& +\frac{\left(a+\lambda \digamma_{5}(\widehat{Z})\right)}{\theta}\left(H_{n+1}-H_{n}-\int_{H_{n}}^{H_{n}+1} \frac{\digamma_{3}(\widehat{H})}{\digamma_{3}(\varsigma)} d \varsigma\right) \\
& \left.+\frac{d\left(a+\lambda \digamma_{5}(\widehat{Z})\right)}{q \theta}\left(Y_{n+1}-Y_{n}\right)+\frac{\lambda e^{-\mu_{3} \tau_{3}}}{g}\left(Z_{n+1}-Z_{n}-\int_{Z_{n}}^{Z_{n+1}} \frac{\digamma_{5}(\widehat{Z})}{\digamma_{5}(\varsigma)} d \varsigma\right)\right] \\
& +\frac{c}{\theta}\left(a+\lambda \digamma_{5}(\widehat{Z})\right) \digamma_{3}(\widehat{H})\left[G\left(\frac{\digamma_{3}\left(H_{n+1}\right)}{\digamma_{3}(\widehat{H})}\right)-G\left(\frac{\digamma_{3}\left(H_{n}\right)}{\digamma_{3}(\widehat{H})}\right)\right] \\
& +\frac{m(1-\varepsilon) e^{-\mu_{1} \tau_{1}-\mu_{3} \tau_{3}}}{\alpha+m} \Lambda(\widehat{F}, \widehat{H})\left(\sum_{j=n-m_{1}+1}^{n} G\left(\frac{\Lambda\left(F_{j+1}, H_{j}\right)}{\Lambda(\widehat{F}, \widehat{H})}\right)\right. \\
& \left.-\sum_{j=n-m_{1}}^{n-1} G\left(\frac{\Lambda\left(F_{j+1}, H_{j}\right)}{\Lambda(\widehat{F}, \widehat{H})}\right)\right) \\
& +\varepsilon e^{-\mu_{2} \tau_{2}-\mu_{3} \tau_{3}} \Lambda(\widehat{F}, \widehat{H})\left(\sum_{j=n-m_{2}+1}^{n} G\left(\frac{\Lambda\left(F_{j+1}, H_{j}\right)}{\Lambda(\widehat{F}, \widehat{H})}\right)-\sum_{j=n-m_{2}}^{n-1} G\left(\frac{\Lambda\left(F_{j+1}, H_{j}\right)}{\Lambda(\widehat{F}, \widehat{H})}\right)\right) \\
& +\gamma \Lambda(\widehat{F}, \widehat{H})\left(\sum_{j=n-m_{3}+1}^{n} G\left(\frac{\digamma_{2}\left(S_{j+1}\right)}{\digamma_{2}(\widehat{S})}\right)-\sum_{j=n-m_{3}}^{n-1} G\left(\frac{\digamma_{2}\left(S_{j+1}\right)}{\digamma_{2}(\widehat{S})}\right)\right) .
\end{aligned}
$$

Using inequalities (45) and (46) by replacing $F^{*}, H^{*}, \rho^{*}$ with $\widehat{F}, \widehat{H}$, $\widehat{\rho}$, we get

$$
\begin{aligned}
\Delta \mathcal{M}_{n} \leq & \frac{1}{\phi(h)}\left[\gamma\left(1-\frac{\Lambda(\widehat{F}, \widehat{H})}{\Lambda\left(F_{n+1}, \widehat{H}\right)}\right)\left(F_{n+1}-F_{n}\right)\right. \\
& +\frac{m e^{-\mu_{3} \tau_{3}}}{\alpha+m}\left(1-\frac{\digamma_{1}(\widehat{K})}{\digamma_{1}\left(K_{n+1}\right)}\right)\left(K_{n+1}-K_{n}\right) \\
& +e^{-\mu_{3} \tau_{3}}\left(1-\frac{\digamma_{2}(\widehat{S})}{\digamma_{2}\left(S_{n+1}\right)}\right)\left(S_{n+1}-S_{n}\right) \\
& +\frac{\left(a+\lambda \digamma_{5}(\widehat{Z})\right)}{\theta}\left(1-\frac{\digamma_{3}(\widehat{H})}{\digamma_{3}\left(H_{n+1}\right)}\right)\left(H_{n+1}-H_{n}\right) \\
& \left.+\frac{d\left(a+\lambda \digamma_{5}(\widehat{Z})\right)}{q \theta}\left(Y_{n+1}-Y_{n}\right)+\frac{\lambda e^{-\mu_{3} \tau_{3}}}{g}\left(1-\frac{\digamma_{5}(\widehat{Z})}{\digamma_{5}\left(Z_{n+1}\right)}\right)\left(Z_{n+1}-Z_{n}\right)\right] \\
& +\frac{c}{\theta}\left(a+\lambda \digamma_{5}(\widehat{Z})\right) \digamma_{3}(\widehat{H})\left[\frac{\digamma_{3}\left(H_{n+1}\right)}{\digamma_{3}(\widehat{H})}-\frac{\digamma_{3}\left(H_{n}\right)}{\digamma_{3}(\widehat{H})}+\ln \left(\frac{\digamma_{3}\left(H_{n}\right)}{\digamma_{3}\left(H_{n+1}\right)}\right)\right] \\
& +\frac{m(1-\varepsilon) e^{-\mu_{1} \tau_{1}-\mu_{3} \tau_{3}}}{\alpha+m} \Lambda(\widehat{F}, \widehat{H})\left[\frac{\Lambda\left(F_{n+1}, H_{n}\right)}{\Lambda(\widehat{F}, \widehat{H})}-\frac{\Lambda\left(F_{n-m_{1}+1}, H_{n-m_{1}}\right)}{\Lambda(\widehat{F}, \widehat{H})}\right. \\
& \left.+\ln \left(\frac{\Lambda\left(F_{n-m_{1}+1}, H_{n-m_{1}}\right)}{\Lambda\left(F_{n+1}, H_{n}\right)}\right)\right] \\
& +\varepsilon e^{-\mu_{2} \tau_{2}-\mu_{3} \tau_{3}} \Lambda(\widehat{F}, \widehat{H})\left[\frac{\Lambda\left(F_{n+1}, H_{n}\right)}{\Lambda(\widehat{F}, \widehat{H})}-\frac{\Lambda\left(F_{n-m_{2}+1}, H_{n-m_{2}}\right)}{\Lambda(\widehat{F}, \widehat{H})}\right.
\end{aligned}
$$




$$
\begin{aligned}
& \left.+\ln \left(\frac{\Lambda\left(F_{n-m_{2}+1}, H_{n-m_{2}}\right)}{\Lambda\left(F_{n+1}, H_{n}\right)}\right)\right] \\
& +\gamma \Lambda(\widehat{F}, \widehat{H})\left[\frac{\digamma_{2}\left(S_{n+1}\right)}{\digamma_{2}(\widehat{S})}-\frac{\digamma_{2}\left(S_{n-m_{3}+1}\right)}{\digamma_{2}(\widehat{S})}+\ln \left(\frac{\digamma_{2}\left(S_{n-m_{3}+1}\right)}{\digamma_{2}\left(S_{n+1}\right)}\right)\right] .
\end{aligned}
$$

From Eqs. (11)-(16) we have

$$
\begin{aligned}
& \Delta \mathcal{M}_{n} \leq \gamma\left(1-\frac{\Lambda(\widehat{F}, \widehat{H})}{\Lambda\left(F_{n+1}, \widehat{H}\right)}\right)\left(\Theta\left(F_{n+1}\right)-\Lambda\left(F_{n+1}, H_{n}\right)\right) \\
& +\frac{m e^{-\mu_{3} \tau_{3}}}{\alpha+m}\left(1-\frac{\digamma_{1}(\widehat{K})}{\digamma_{1}\left(K_{n+1}\right)}\right)\left[(1-\varepsilon) e^{-\mu_{1} \tau_{1}} \Lambda\left(F_{n-m_{1}+1}, H_{n-m_{1}}\right)\right. \\
& \left.-(\alpha+m) \digamma_{1}\left(K_{n+1}\right)\right] \\
& +e^{-\mu_{3} \tau_{3}}\left(1-\frac{\digamma_{2}(\widehat{S})}{\digamma_{2}\left(S_{n+1}\right)}\right)\left[\varepsilon e^{-\mu_{2} \tau_{2}} \Lambda\left(F_{n-m_{2}+1}, H_{n-m_{2}}\right)+m \digamma_{1}\left(K_{n+1}\right)\right. \\
& \left.-a \digamma_{2}\left(S_{n+1}\right)-\lambda \digamma_{2}\left(S_{n+1}\right) \digamma_{5}\left(Z_{n+1}\right)\right] \\
& +\frac{\left(a+\lambda \digamma_{5}(\widehat{Z})\right)}{\theta}\left(1-\frac{\digamma_{3}(\widehat{H})}{\digamma_{3}\left(H_{n+1}\right)}\right)\left[\theta e^{-\mu_{3} \tau_{3}} \digamma_{2}\left(S_{n-m_{3}+1}\right)-c \digamma_{3}\left(H_{n+1}\right)\right. \\
& \left.-d \digamma_{3}\left(H_{n+1}\right) \digamma_{4}\left(Y_{n+1}\right)\right] \\
& +\frac{d\left(a+\lambda \digamma_{5}(\widehat{Z})\right)}{q \theta}\left[q \digamma_{3}\left(H_{n+1}\right) \digamma_{4}\left(Y_{n+1}\right)-\eta \digamma_{4}\left(Y_{n+1}\right)\right] \\
& +\frac{\lambda e^{-\mu_{3} \tau_{3}}}{g}\left(1-\frac{\digamma_{5}(\widehat{Z})}{\digamma_{5}\left(Z_{n+1}\right)}\right)\left[g \digamma_{2}\left(S_{n+1}\right) \digamma_{5}\left(Z_{n+1}\right)-\xi \digamma_{5}\left(Z_{n+1}\right)\right] \\
& +\frac{c}{\theta}\left(a+\lambda \digamma_{5}(\widehat{Z})\right) \digamma_{3}(\widehat{H})\left[\frac{\digamma_{3}\left(H_{n+1}\right)}{\digamma_{3}(\widehat{H})}-\frac{\digamma_{3}\left(H_{n}\right)}{\digamma_{3}(\widehat{H})}+\ln \left(\frac{\digamma_{3}\left(H_{n}\right)}{\digamma_{3}\left(H_{n+1}\right)}\right)\right] \\
& +\frac{m(1-\varepsilon) e^{-\mu_{1} \tau_{1}-\mu_{3} \tau_{3}}}{\alpha+m} \Lambda(\widehat{F}, \widehat{H})\left[\frac{\Lambda\left(F_{n+1}, H_{n}\right)}{\Lambda(\widehat{F}, \widehat{H})}-\frac{\Lambda\left(F_{n-m_{1}+1}, H_{n-m_{1}}\right)}{\Lambda(\widehat{F}, \widehat{H})}\right. \\
& \left.+\ln \left(\frac{\Lambda\left(F_{n-m_{1}+1}, H_{n-m_{1}}\right)}{\Lambda\left(F_{n+1}, H_{n}\right)}\right)\right] \\
& +\varepsilon e^{-\mu_{2} \tau_{2}-\mu_{3} \tau_{3}} \Lambda(\widehat{F}, \widehat{H})\left[\frac{\Lambda\left(F_{n+1}, H_{n}\right)}{\Lambda(\widehat{F}, \widehat{H})}-\frac{\Lambda\left(F_{n-m_{2}+1}, H_{n-m_{2}}\right)}{\Lambda(\widehat{F}, \widehat{H})}\right. \\
& \left.+\ln \left(\frac{\Lambda\left(F_{n-m_{2}+1}, H_{n-m_{2}}\right)}{\Lambda\left(F_{n+1}, H_{n}\right)}\right)\right] \\
& +\gamma \Lambda(\widehat{F}, \widehat{H})\left[\frac{\digamma_{2}\left(S_{n+1}\right)}{\digamma_{2}(\widehat{S})}-\frac{\digamma_{2}\left(S_{n-m_{3}+1}\right)}{\digamma_{2}(\widehat{S})}+\ln \left(\frac{\digamma_{2}\left(S_{n-m_{3}+1}\right)}{\digamma_{2}\left(S_{n+1}\right)}\right)\right] .
\end{aligned}
$$

Collecting terms of Eq. (55), we get

$$
\begin{aligned}
\Delta \mathcal{M}_{n} \leq & \gamma\left(1-\frac{\Lambda(\widehat{F}, \widehat{H})}{\Lambda\left(F_{n+1}, \widehat{H}\right)}\right)\left(\Theta\left(F_{n+1}\right)-\Theta(\widehat{F})\right)+\gamma \Theta(\widehat{F})\left(1-\frac{\Lambda(\widehat{F}, \widehat{H})}{\Lambda\left(F_{n+1}, \widehat{H}\right)}\right) \\
& +\gamma \frac{\Lambda(\widehat{F}, \widehat{H})}{\Lambda\left(F_{n+1}, \widehat{H}\right)} \Lambda\left(F_{n+1}, H_{n}\right) \\
& -\frac{m(1-\varepsilon) e^{-\mu_{1} \tau_{1}-\mu_{3} \tau_{3}}}{\alpha+m} \Lambda\left(F_{n-m_{1}+1}, H_{n-m_{1}}\right) \frac{\digamma_{1}(\widehat{K})}{\digamma_{1}\left(K_{n+1}\right)}
\end{aligned}
$$




$$
\begin{aligned}
& +m e^{-\mu_{3} \tau_{3}} \digamma_{1}(\widehat{K})-\varepsilon e^{-\mu_{2} \tau_{2}-\mu_{3} \tau_{3}} \Lambda\left(F_{n-m_{2}+1}, H_{n-m_{2}}\right) \frac{\digamma_{2}(\widehat{S})}{\digamma_{2}\left(S_{n+1}\right)} \\
& -m e^{-\mu_{3} \tau_{3}} \digamma_{1}\left(K_{n+1}\right) \frac{\digamma_{2}(\widehat{S})}{\digamma_{2}\left(S_{n+1}\right)} \\
& -a e^{-\mu_{3} \tau_{3}} \digamma_{2}\left(S_{n+1}\right)+\lambda e^{-\mu_{3} \tau_{3}} \digamma_{2}(\widehat{S}) \digamma_{5}\left(Z_{n+1}\right)+a e^{-\mu_{3} \tau_{3}} \digamma_{2}(\widehat{S}) \\
& +\left(a+\lambda \digamma_{5}(\widehat{Z})\right) e^{-\mu_{3} \tau_{3}} \digamma_{2}\left(S_{n-m_{3}+1}\right) \\
& -\left(a+\lambda \digamma_{5}(\widehat{Z})\right) e^{-\mu_{3} \tau_{3}} \digamma_{2}\left(S_{n-m_{3}+1}\right) \frac{\digamma_{3}(\widehat{H})}{\digamma_{3}\left(H_{n+1}\right)} \\
& +\frac{c\left(a+\lambda \digamma_{5}(\widehat{Z})\right)}{\theta} \digamma_{3}(\widehat{H})+\frac{d\left(a+\lambda \digamma_{5}(\widehat{Z})\right)}{\theta} \digamma_{3}(\widehat{H}) \digamma_{4}\left(Y_{n+1}\right) \\
& -\frac{d\left(a+\lambda \digamma_{5}(\widehat{Z})\right) \eta}{q \theta} \digamma_{4}\left(Y_{n+1}\right) \\
& -\lambda e^{-\mu_{3} \tau_{3}} \digamma_{5}(\widehat{Z}) \digamma_{2}\left(S_{n+1}\right)-\frac{\lambda e^{-\mu_{3} \tau_{3}} \xi}{g} \digamma_{5}\left(Z_{n+1}\right)+\frac{\lambda e^{-\mu_{3} \tau_{3}} \xi}{g} \digamma_{5}(\widehat{Z}) \\
& -\frac{c}{\theta}\left(a+\lambda \digamma_{5}(\widehat{Z})\right) \digamma_{3}\left(H_{n}\right)+\frac{c}{\theta}\left(a+\lambda \digamma_{5}(\widehat{Z})\right) \digamma_{3}(\widehat{H}) \ln \left(\frac{\digamma_{3}\left(H_{n}\right)}{\digamma_{3}\left(H_{n+1}\right)}\right) \\
& +\frac{m(1-\varepsilon) e^{-\mu_{1} \tau_{1}-\mu_{3} \tau_{3}}}{\alpha+m} \Lambda(\widehat{F}, \widehat{H}) \ln \left(\frac{\Lambda\left(F_{n-m_{1}+1}, H_{n-m_{1}}\right)}{\Lambda\left(F_{n+1}, H_{n}\right)}\right) \\
& +\varepsilon e^{-\mu_{2} \tau_{2}-\mu_{3} \tau_{3}} \Lambda(\widehat{F}, \widehat{H}) \ln \left(\frac{\Lambda\left(F_{n-m_{2}+1}, H_{n-m_{2}}\right)}{\Lambda\left(F_{n+1}, H_{n}\right)}\right) \\
& +\gamma \Lambda(\widehat{F}, \widehat{H})\left(\frac{\digamma_{2}\left(S_{n+1}\right)}{\digamma_{2}(\widehat{S})}-\frac{\digamma_{2}\left(S_{n-m_{3}+1}\right)}{\digamma_{2}(\widehat{S})}+\ln \frac{\digamma_{2}\left(S_{n-m_{3}+1}\right)}{\digamma_{2}\left(S_{n+1}\right)}\right) \text {. }
\end{aligned}
$$

Using the conditions of $\widehat{Q}$

$$
\begin{aligned}
& \Theta(\widehat{F})=\Lambda(\widehat{F}, \widehat{H}), \\
& (1-\varepsilon) e^{-\mu_{1} \tau_{1}} \Lambda(\widehat{F}, \widehat{H})=(\alpha+m) \digamma_{1}(\widehat{K}), \\
& \varepsilon e^{-\mu_{2} \tau_{2}-\mu_{3} \tau_{3}} \Lambda(\widehat{F}, \widehat{H})+m e^{-\mu_{3} \tau_{3}} \digamma_{1}(\widehat{K})=\gamma \Lambda(\widehat{F}, \widehat{H})=\left(a+\lambda \digamma_{5}(\widehat{Z})\right) e^{-\mu_{3} \tau_{3}} \digamma_{2}(\widehat{S}), \\
& \theta e^{-\mu_{3} \tau_{3}} \digamma_{2}(\widehat{S})=c \digamma_{3}(\widehat{H}), \\
& \digamma_{2}(\widehat{S})=\frac{\xi}{g}
\end{aligned}
$$

we get

$$
\begin{aligned}
\Delta \mathcal{M}_{n} \leq & \gamma\left(1-\frac{\Lambda(\widehat{F}, \widehat{H})}{\Lambda\left(F_{n+1}, \widehat{H}\right)}\right)\left(\Theta\left(F_{n+1}\right)-\Theta(\widehat{F})\right)+\gamma \Lambda(\widehat{F}, \widehat{H})\left(1-\frac{\Lambda(\widehat{F}, \widehat{H})}{\Lambda\left(F_{n+1}, \widehat{H}\right)}\right) \\
& +\gamma \Lambda(\widehat{F}, \widehat{H}) \frac{\Lambda\left(F_{n+1}, H_{n}\right)}{\Lambda\left(F_{n+1}, \widehat{H}\right)} \\
& -\frac{m(1-\varepsilon) e^{-\mu_{1} \tau_{1}-\mu_{3} \tau_{3}}}{\alpha+m} \Lambda(\widehat{F}, \widehat{H}) \frac{\Lambda\left(F_{n-m_{1}+1}, H_{n-m_{1}}\right) \digamma_{1}(\widehat{K})}{\Lambda(\widehat{F}, \widehat{H}) \digamma_{1}\left(K_{n+1}\right)} \\
& +\frac{m(1-\varepsilon) e^{-\mu_{1} \tau_{1}-\mu_{3} \tau_{3}}}{\alpha+m} \Lambda(\widehat{F}, \widehat{H})
\end{aligned}
$$




$$
\begin{aligned}
& -\varepsilon e^{-\mu_{2} \tau_{2}-\mu_{3} \tau_{3}} \Lambda(\widehat{F}, \widehat{H}) \frac{\Lambda\left(F_{n-m_{2}+1}, H_{n-m_{2}}\right) \digamma_{2}(\widehat{S})}{\Lambda(\widehat{F}, \widehat{H}) \digamma_{2}\left(S_{n+1}\right)} \\
& -\frac{m(1-\varepsilon) e^{-\mu_{1} \tau_{1}-\mu_{3} \tau_{3}}}{\alpha+m} \Lambda(\widehat{F}, \widehat{H}) \frac{\digamma_{1}\left(K_{n+1}\right) \digamma_{2}(\widehat{S})}{\digamma_{1}(\widehat{K}) \digamma_{2}\left(S_{n+1}\right)}+\gamma \Lambda(\widehat{F}, \widehat{H}) \\
& -\gamma \Lambda(\widehat{F}, \widehat{H}) \frac{\digamma_{3}(\widehat{H}) \digamma_{2}\left(S_{n-m_{3}+1}\right)}{\digamma_{3}\left(H_{n+1}\right) \digamma_{2}(\widehat{S})} \\
& +\gamma \Lambda(\widehat{F}, \widehat{H})-\gamma \Lambda(\widehat{F}, \widehat{H}) \frac{\digamma_{3}\left(H_{n}\right)}{\digamma_{3}(\widehat{H})}+\frac{d\left(a+\lambda \digamma_{5}(\widehat{Z})\right)}{\theta}\left(\digamma_{3}(\widehat{H})-\frac{\eta}{q}\right) \digamma_{4}\left(Y_{n+1}\right) \\
& +\gamma \Lambda(\widehat{F}, \widehat{H}) \ln \left(\frac{\digamma_{3}\left(H_{n}\right)}{\digamma_{3}\left(H_{n+1}\right)}\right) \\
& +\frac{m(1-\varepsilon) e^{-\mu_{1} \tau_{1}-\mu_{3} \tau_{3}}}{\alpha+m} \Lambda(\widehat{F}, \widehat{H}) \ln \left(\frac{\Lambda\left(F_{n-m_{1}+1}, H_{n-m_{1}}\right)}{\Lambda\left(F_{n+1}, H_{n}\right)}\right) \\
& +\varepsilon e^{-\mu_{2} \tau_{2}-\mu_{3} \tau_{3}} \Lambda(\widehat{F}, \widehat{H}) \ln \left(\frac{\Lambda\left(F_{n-m_{2}+1}, H_{n-m_{2}}\right)}{\Lambda\left(F_{n+1}, H_{n}\right)}\right) \\
& +\gamma \Lambda(\widehat{F}, \widehat{H}) \ln \left(\frac{\digamma_{2}\left(S_{n-m_{3}+1}\right)}{\digamma_{2}\left(S_{n+1}\right)}\right) \\
& =\gamma\left(1-\frac{\Lambda(\widehat{F}, \widehat{H})}{\Lambda\left(F_{n+1}, \widehat{H}\right)}\right)\left(\Theta\left(F_{n+1}\right)-\Theta(\widehat{F})\right) \\
& +\frac{m(1-\varepsilon) e^{-\mu_{1} \tau_{1}-\mu_{3} \tau_{3}}}{\alpha+m} \Lambda(\widehat{F}, \widehat{H})\left[5-\frac{\Lambda(\widehat{F}, \widehat{H})}{\Lambda\left(F_{n+1}, \widehat{H}\right)}\right. \\
& -\frac{\Lambda\left(F_{n-m_{1}+1}, H_{n-m_{1}}\right) \digamma_{1}(\widehat{K})}{\Lambda(\widehat{F}, \widehat{H}) \digamma_{1}\left(K_{n+1}\right)}-\frac{\digamma_{1}\left(K_{n+1}\right) \digamma_{2}(\widehat{S})}{\digamma_{1}(\widehat{K}) \digamma_{2}\left(S_{n+1}\right)} \\
& -\frac{\digamma_{3}(\widehat{H}) \digamma_{2}\left(S_{n-m_{3}+1}\right)}{\digamma_{3}\left(H_{n+1}\right) \digamma_{2}(\widehat{S})}-\frac{\Lambda\left(F_{n+1}, \widehat{H}\right) \digamma_{3}\left(H_{n}\right)}{\Lambda\left(F_{n+1}, H_{n}\right) \digamma_{3}(\widehat{H})} \\
& \left.+\ln \left(\frac{\Lambda\left(F_{n-m_{1}+1}, H_{n-m_{1}}\right) \digamma_{2}\left(S_{n-m_{3}+1}\right) \digamma_{3}\left(H_{n}\right)}{\Lambda\left(F_{n+1}, H_{n}\right) \digamma_{2}\left(S_{n+1}\right) \digamma_{3}\left(H_{n+1}\right)}\right)\right] \\
& +\varepsilon e^{-\mu_{2} \tau_{2}-\mu_{3} \tau_{3}} \Lambda(\widehat{F}, \widehat{H})\left[4-\frac{\Lambda(\widehat{F}, \widehat{H})}{\Lambda\left(F_{n+1}, \widehat{H}\right)}-\frac{\Lambda\left(F_{n-m_{2}+1}, H_{n-m_{2}}\right) \digamma_{2}(\widehat{S})}{\Lambda(\widehat{F}, \widehat{H}) \digamma_{2}\left(S_{n+1}\right)}\right. \\
& -\frac{\digamma_{3}(\widehat{H}) \digamma_{2}\left(S_{n-m_{3}+1}\right)}{\digamma_{3}\left(H_{n+1}\right) \digamma_{2}(\widehat{S})} \\
& \left.-\frac{\Lambda\left(F_{n+1}, \widehat{H}\right) \digamma_{3}\left(H_{n}\right)}{\Lambda\left(F_{n+1}, H_{n}\right) \digamma_{3}(\widehat{H})}+\ln \left(\frac{\Lambda\left(F_{n-m_{2}+1}, H_{n-m_{2}}\right) \digamma_{2}\left(S_{n-m_{3}+1}\right) \digamma_{3}\left(H_{n}\right)}{\Lambda\left(F_{n+1}, H_{n}\right) \digamma_{2}\left(S_{n+1}\right) \digamma_{3}\left(H_{n+1}\right)}\right)\right] \\
& +\frac{d\left(a+\lambda \digamma_{5}(\widehat{Z})\right)}{\theta}\left(\digamma_{3}(\widehat{H})-\digamma_{3}(\widetilde{H})\right) \digamma_{4}\left(Y_{n+1}\right) \\
& +\gamma \Lambda(\widehat{F}, \widehat{H})\left[-1+\frac{\Lambda\left(F_{n+1}, \widehat{H}\right) \digamma_{3}\left(H_{n}\right)}{\Lambda\left(F_{n+1}, H_{n}\right) \digamma_{3}(\widehat{H})}+\frac{\Lambda\left(F_{n+1}, H_{n}\right)}{\Lambda\left(F_{n+1}, \widehat{H}\right)}-\frac{\digamma_{3}\left(H_{n}\right)}{\digamma_{3}(\widehat{H})}\right], \\
& \Delta \mathcal{M}_{n} \leq \gamma\left(1-\frac{\Lambda(\widehat{F}, \widehat{H})}{\Lambda\left(F_{n+1}, \widehat{H}\right)}\right)\left(\Theta\left(F_{n+1}\right)-\Theta(\widehat{F})\right) \\
& -\frac{m(1-\varepsilon) e^{-\mu_{1} \tau_{1}-\mu_{3} \tau_{3}}}{\alpha+m} \Lambda(\widehat{F}, \widehat{H})\left[G\left(\frac{\Lambda(\widehat{F}, \widehat{H})}{\Lambda\left(F_{n+1}, \widehat{H}\right)}\right)\right. \\
& +G\left(\frac{\Lambda\left(F_{n-m_{1}+1}, H_{n-m_{1}}\right) \digamma_{1}(\widehat{K})}{\Lambda(\widehat{F}, \widehat{H}) \digamma_{1}\left(K_{n+1}\right)}\right)+G\left(\frac{\digamma_{1}\left(K_{n+1}\right) \digamma_{2}(\widehat{S})}{\digamma_{1}(\widehat{K}) \digamma_{2}\left(S_{n+1}\right)}\right)
\end{aligned}
$$




$$
\begin{aligned}
& +G\left(\frac{\digamma_{3}(\widehat{H}) \digamma_{2}\left(S_{n-m_{3}+1}\right)}{\digamma_{3}\left(H_{n+1}\right) \digamma_{2}(\widehat{S})}\right) \\
& \left.+G\left(\frac{\Lambda\left(F_{n+1}, \widehat{H}\right) \digamma_{3}\left(H_{n}\right)}{\Lambda\left(F_{n+1}, H_{n}\right) \digamma_{3}(\widehat{H})}\right)\right]-\varepsilon e^{-\mu_{2} \tau_{2}-\mu_{3} \tau_{3}} \Lambda(\widehat{F}, \widehat{H})\left[G\left(\frac{\Lambda(\widehat{F}, \widehat{H})}{\Lambda\left(F_{n+1}, \widehat{H}\right)}\right)\right. \\
& +G\left(\frac{\Lambda\left(F_{n-m_{2}+1}, H_{n-m_{2}}\right) \digamma_{2}(\widehat{S})}{\Lambda(\widehat{F}, \widehat{H}) \digamma_{2}\left(S_{n+1}\right)}\right)+G\left(\frac{\digamma_{3}(\widehat{H}) \digamma_{2}\left(S_{n-m_{3}+1}\right)}{\digamma_{3}\left(H_{n+1}\right) \digamma_{2}(\widehat{S})}\right) \\
& \left.+G\left(\frac{\Lambda\left(F_{n+1}, \widehat{H}\right) \digamma_{3}\left(H_{n}\right)}{\Lambda\left(F_{n+1}, H_{n}\right) \digamma_{3}(\widehat{H})}\right)\right] \\
& +\frac{d\left(a+\lambda \digamma_{5}(\widehat{Z})\right)}{\theta} \digamma_{3}(\widetilde{H})\left(\mathcal{R}_{1}^{Y} / \mathcal{R}_{2}^{Z}-1\right) \digamma_{4}\left(Y_{n+1}\right) \\
& +\gamma \Lambda(\widehat{F}, \widehat{H})\left(1-\frac{\Lambda\left(F_{n+1}, \widehat{H}\right)}{\Lambda\left(F_{n+1}, H_{n}\right)}\right)\left(\frac{\Lambda\left(F_{n+1}, H_{n}\right)}{\Lambda\left(F_{n+1}, \widehat{H}\right)}-\frac{\digamma_{3}\left(H_{n}\right)}{\digamma_{3}(\widehat{H})}\right) .
\end{aligned}
$$

Using Conditions $\mathrm{C} 1-\mathrm{C} 4$, we get that the first and last terms of Eq. (56) are less than or equal to zero. Moreover, if $\mathcal{R}_{1}^{Y} / \mathcal{R}_{2}^{Z} \leq 1$, we get $\Delta \mathcal{M}_{n} \leq 0$, and thus $\mathcal{M}_{n}$ is a monotone decreasing sequence. Since $\mathcal{M}_{n} \geq 0$, then there is a $\operatorname{limit}_{n \rightarrow \infty} \lim _{n} \geq 0$. Therefore, $\lim _{n \rightarrow \infty} \Delta \mathcal{M}_{n}=0$, which implies that $\lim _{n \rightarrow \infty} F_{n}=\widehat{F}, \lim _{n \rightarrow \infty} K_{n}=\widehat{K}, \lim _{n \rightarrow \infty} S_{n}=\widehat{S}$, $\lim _{n \rightarrow \infty} H_{n}=\widehat{H}$, and $\lim _{n \rightarrow \infty}\left(\mathcal{R}_{1}^{Y} / \mathcal{R}_{2}^{Z}-1\right) Y_{n+1}=0$. We have two cases as follows:

- $\mathcal{R}_{1}^{Y} / \mathcal{R}_{2}^{Z}=1$, from Eq. (13)

$$
0=\varepsilon e^{-\mu_{2} \tau_{2}} \Lambda(\widehat{F}, \widehat{H})+m \digamma_{1}(\widehat{K})-a \digamma_{2}(\widehat{S})-\lambda \digamma_{2}(\widehat{S}) \lim _{n \rightarrow \infty} \digamma_{5}\left(Z_{n+1}\right)
$$

and this gives $\lim _{n \rightarrow \infty} Z_{n}=\widehat{Z}$. Moreover, from Eq. (14) we have

$$
0=\theta e^{-\mu_{3} \tau_{3}} \digamma_{2}(\widehat{S})-c \digamma_{3}(\widehat{H})-d \digamma_{3}(\widehat{H}) \lim _{n \rightarrow \infty} \digamma_{4}\left(Y_{n+1}\right),
$$

then we get $\lim _{n \rightarrow \infty} Y_{n}=0$.

- $\mathcal{R}_{1}^{Y} / \mathcal{R}_{2}^{Z}<1, \lim _{n \rightarrow \infty} Y_{n}=0$. From Eq. (57) we get $\lim _{n \rightarrow \infty} Z_{n}=\widehat{Z}$. Then we get that $\widehat{Q}$ is G.A.S.

Proof of Theorem 5 Define $\mathcal{V}_{n}\left(F_{n}, K_{n}, S_{n}, H_{n}, Y_{n}, Z_{n}\right)$ :

$$
\begin{aligned}
\mathcal{V}_{n}= & \frac{1}{\phi(h)}\left[\gamma\left(F_{n}-\widetilde{F}-\int_{\widetilde{F}}^{F_{n}} \frac{\Lambda(\widetilde{F}, \widetilde{H})}{\Lambda(\varsigma, \widetilde{H})} d \varsigma\right)+\frac{m e^{-\mu_{3} \tau_{3}}}{\alpha+m}\left(K_{n}-\widetilde{K}-\int_{\widetilde{K}}^{K_{n}} \frac{\digamma_{1}(\widetilde{K})}{\digamma_{1}(\varsigma)} d \varsigma\right)\right. \\
& +e^{-\mu_{3} \tau_{3}}\left(S_{n}-\widetilde{S}-\int_{\widetilde{S}}^{S_{n}} \frac{\digamma_{2}(\widetilde{S})}{\digamma_{2}(\varsigma)} d \varsigma\right) \\
& +\frac{\left(a+\lambda \digamma_{5}(\widetilde{Z})\right)}{\theta}\left(H_{n}-\widetilde{H}-\int_{\widetilde{H}}^{H_{n}} \frac{\digamma_{3}(\widetilde{H})}{\digamma_{3}(\varsigma)} d \varsigma\right) \\
& +\frac{d\left(a+\lambda \digamma_{5}(\widetilde{Z})\right)}{q \theta}\left(Y_{n}-\widetilde{Y}-\int_{\widetilde{Y}}^{Y_{n}} \frac{\digamma_{4}(\widetilde{Y})}{\digamma_{4}(\varsigma)} d \varsigma\right) \\
& \left.+\frac{\lambda e^{-\mu_{3} \tau_{3}}}{g}\left(Z_{n}-\widetilde{Z}-\int_{\widetilde{Z}}^{Z_{n}} \frac{\digamma_{5}(\widetilde{Z})}{\digamma_{5}(\varsigma)} d \varsigma\right)\right] \\
& +\frac{\left(c+d \digamma_{4}(\widetilde{Y})\right)}{\theta}\left(a+\lambda \digamma_{5}(\widetilde{Z})\right) \digamma_{3}(\widetilde{H}) G\left(\frac{\digamma_{3}\left(H_{n}\right)}{\digamma_{3}(\widetilde{H})}\right)
\end{aligned}
$$




$$
\begin{aligned}
& +\frac{m(1-\varepsilon) e^{-\mu_{1} \tau_{1}-\mu_{3} \tau_{3}}}{\alpha+m} \Lambda(\widetilde{F}, \widetilde{H}) \sum_{j=n-m_{1}}^{n-1} G\left(\frac{\Lambda\left(F_{j+1}, H_{j}\right)}{\Lambda(\widetilde{F}, \widetilde{H})}\right) \\
& +\varepsilon e^{-\mu_{2} \tau_{2}-\mu_{3} \tau_{3}} \Lambda(\widetilde{F}, \widetilde{H}) \sum_{j=n-m_{2}}^{n-1} G\left(\frac{\Lambda\left(F_{j+1}, H_{j}\right)}{\Lambda(\widetilde{F}, \widetilde{H})}\right)+\gamma \Lambda(\widetilde{F}, \widetilde{H}) \sum_{j=n-m_{3}}^{n-1} G\left(\frac{\digamma_{2}\left(S_{j+1}\right)}{\digamma_{2}(\widetilde{S})}\right)
\end{aligned}
$$

Clearly, $\mathcal{V}_{n}\left(F_{n}, K_{n}, S_{n}, H_{n}, Y_{n}, Z_{n}\right)>0$ for all $F_{n}, K_{n}, S_{n}, H_{n}, Y_{n}, Z_{n}>0$ and $\mathcal{V}_{n}(\widetilde{F}, \widetilde{K}, \widetilde{S}, \widetilde{H}, \widetilde{Y}$, $\widetilde{Z})=0$. We compute $\Delta \mathcal{V}_{n}=\mathcal{V}_{n+1}-\mathcal{V}_{n}$ as follows:

$$
\begin{aligned}
& \Delta \mathcal{V}_{n}=\frac{1}{\phi(h)}\left[\gamma\left(F_{n+1}-\widetilde{F}-\int_{\widetilde{F}}^{F_{n+1}} \frac{\Lambda(\widetilde{F}, \widetilde{H})}{\Lambda(\varsigma, \widetilde{H})} d \varsigma\right)\right. \\
& +\frac{m e^{-\mu_{3} \tau_{3}}}{\alpha+m}\left(K_{n+1}-\widetilde{K}-\int_{\widetilde{K}}^{K_{n+1}} \frac{\digamma_{1}(\widetilde{K})}{\digamma_{1}(\varsigma)} d \varsigma\right) \\
& +e^{-\mu_{3} \tau_{3}}\left(S_{n+1}-\widetilde{S}-\int_{\widetilde{S}}^{S_{n+1}} \frac{\digamma_{2}(\widetilde{S})}{\digamma_{2}(\varsigma)} d \varsigma\right) \\
& +\frac{\left(a+\lambda \digamma_{5}(\widetilde{Z})\right)}{\theta}\left(H_{n+1}-\widetilde{H}-\int_{\widetilde{H}}^{H_{n+1}} \frac{\digamma_{3}(\widetilde{H})}{\digamma_{3}(\varsigma)} d \varsigma\right) \\
& +\frac{d\left(a+\lambda \digamma_{5}(\widetilde{Z})\right)}{q \theta}\left(Y_{n+1}-\tilde{Y}-\int_{\widetilde{Y}}^{Y_{n+1}} \frac{\digamma_{4}(\tilde{Y})}{\digamma_{4}(\varsigma)} d \varsigma\right) \\
& \left.+\frac{\lambda e^{-\mu_{3} \tau_{3}}}{g}\left(Z_{n+1}-\widetilde{Z}-\int_{\tilde{Z}}^{Z_{n+1}} \frac{\digamma_{5}(\widetilde{Z})}{\digamma_{5}(\varsigma)} d \varsigma\right)\right] \\
& +\frac{\left(c+d \digamma_{4}(\tilde{Y})\right)}{\theta}\left(a+\lambda \digamma_{5}(\widetilde{Z})\right) \digamma_{3}(\widetilde{H}) G\left(\frac{\digamma_{3}\left(H_{n+1}\right)}{\digamma_{3}(\widetilde{H})}\right) \\
& +\frac{m(1-\varepsilon) e^{-\mu_{1} \tau_{1}-\mu_{3} \tau_{3}}}{\alpha+m} \Lambda(\widetilde{F}, \widetilde{H}) \sum_{j=n-m_{1}+1}^{n} G\left(\frac{\Lambda\left(F_{j+1}, H_{j}\right)}{\Lambda(\widetilde{F}, \widetilde{H})}\right) \\
& +\varepsilon e^{-\mu_{2} \tau_{2}-\mu_{3} \tau_{3}} \Lambda(\widetilde{F}, \widetilde{H}) \sum_{j=n-m_{2}+1}^{n} G\left(\frac{\Lambda\left(F_{j+1}, H_{j}\right)}{\Lambda(\widetilde{F}, \widetilde{H})}\right) \\
& +\gamma \Lambda(\widetilde{F}, \widetilde{H}) \sum_{j=n-m_{3}+1}^{n} G\left(\frac{\digamma_{2}\left(S_{j+1}\right)}{\digamma_{2}(\widetilde{S})}\right) \\
& -\frac{1}{\phi(h)}\left[\gamma\left(F_{n}-\widetilde{F}-\int_{\widetilde{F}}^{F_{n}} \frac{\Lambda(\widetilde{F}, \widetilde{H})}{\Lambda(\varsigma, \widetilde{H})} d \varsigma\right)+\frac{m e^{-\mu_{3} \tau_{3}}}{\alpha+m}\left(K_{n}-\widetilde{K}-\int_{\widetilde{K}}^{K_{n}} \frac{\digamma_{1}(\widetilde{K})}{\digamma_{1}(\varsigma)} d \varsigma\right)\right. \\
& +e^{-\mu_{3} \tau_{3}}\left(S_{n}-\widetilde{S}-\int_{\widetilde{S}}^{S_{n}} \frac{\digamma_{2}(\widetilde{S})}{\digamma_{2}(\varsigma)} d \varsigma\right)+\frac{\left(a+\lambda \digamma_{5}(\widetilde{Z})\right)}{\theta}\left(H_{n}-\widetilde{H}-\int_{\widetilde{H}}^{H_{n}} \frac{\digamma_{3}(\widetilde{H})}{\digamma_{3}(\varsigma)} d \varsigma\right) \\
& +\frac{d\left(a+\lambda \digamma_{5}(\tilde{Z})\right)}{q \theta}\left(Y_{n}-\widetilde{Y}-\int_{\widetilde{Y}}^{Y_{n}} \frac{\digamma_{4}(\tilde{Y})}{\digamma_{4}(\varsigma)} d \varsigma\right) \\
& \left.+\frac{\lambda e^{-\mu_{3} \tau_{3}}}{g}\left(Z_{n}-\widetilde{Z}-\int_{\widetilde{Z}}^{Z_{n}} \frac{\digamma_{5}(\widetilde{Z})}{\digamma_{5}(\varsigma)} d \varsigma\right)\right] \\
& -\frac{\left(c+d \digamma_{4}(\widetilde{Y})\right)}{\theta}\left(a+\lambda \digamma_{5}(\widetilde{Z})\right) \digamma_{3}(\widetilde{H}) G\left(\frac{\digamma_{3}\left(H_{n}\right)}{\digamma_{3}(\widetilde{H})}\right) \\
& -\frac{m(1-\varepsilon) e^{-\mu_{1} \tau_{1}-\mu_{3} \tau_{3}}}{\alpha+m} \Lambda(\widetilde{F}, \widetilde{H}) \sum_{j=n-m_{1}}^{n-1} G\left(\frac{\Lambda\left(F_{j+1}, H_{j}\right)}{\Lambda(\widetilde{F}, \widetilde{H})}\right)
\end{aligned}
$$




$$
\begin{aligned}
& -\varepsilon e^{-\mu_{2} \tau_{2}-\mu_{3} \tau_{3}} \Lambda(\widetilde{F}, \widetilde{H}) \sum_{j=n-m_{2}}^{n-1} G\left(\frac{\Lambda\left(F_{j+1}, H_{j}\right)}{\Lambda(\widetilde{F}, \widetilde{H})}\right)-\gamma \Lambda(\widetilde{F}, \widetilde{H}) \sum_{j=n-m_{3}}^{n-1} G\left(\frac{\digamma_{2}\left(S_{j+1}\right)}{\digamma_{2}(\widetilde{S})}\right), \\
& \Delta \mathcal{V}_{n}=\frac{1}{\phi(h)}\left[\gamma\left(F_{n+1}-F_{n}-\int_{F_{n}}^{F_{n+1}} \frac{\Lambda(\widetilde{F}, \widetilde{H})}{\Lambda(\varsigma, \widetilde{H})} d \varsigma\right)\right. \\
& +\frac{m e^{-\mu_{3} \tau_{3}}}{\alpha+m}\left(K_{n+1}-K_{n}-\int_{K_{n}}^{K_{n+1}} \frac{\digamma_{1}(\widetilde{K})}{\digamma_{1}(\varsigma)} d \varsigma\right) \\
& +e^{-\mu_{3} \tau_{3}}\left(S_{n+1}-S_{n}-\int_{S_{n}}^{S_{n+1}} \frac{\digamma_{2}(\widetilde{S})}{\digamma_{2}(\varsigma)} d \varsigma\right) \\
& +\frac{\left(a+\lambda \digamma_{5}(\widetilde{Z})\right)}{\theta}\left(H_{n+1}-H_{n}-\int_{H_{n}}^{H_{n}+1} \frac{\digamma_{3}(\tilde{H})}{\digamma_{3}(\varsigma)} d \varsigma\right) \\
& +\frac{d\left(a+\lambda \digamma_{5}(\widetilde{Z})\right)}{q \theta}\left(Y_{n+1}-Y_{n}-\int_{Y_{n}}^{Y_{n+1}} \frac{\digamma_{4}(\widetilde{Y})}{\digamma_{4}(\varsigma)} d \varsigma\right) \\
& \left.+\frac{\lambda e^{-\mu_{3} \tau_{3}}}{g}\left(Z_{n+1}-Z_{n}-\int_{Z_{n}}^{Z_{n+1}} \frac{\digamma_{5}(\widetilde{Z})}{\digamma_{5}(\varsigma)} d \varsigma\right)\right] \\
& +\frac{\left(c+d \digamma_{4}(\widetilde{Y})\right)}{\theta}\left(a+\lambda \digamma_{5}(\widetilde{Z})\right) \digamma_{3}(\widetilde{H})\left[G\left(\frac{\digamma_{3}\left(H_{n+1}\right)}{\digamma_{3}(\widetilde{H})}\right)-G\left(\frac{\digamma_{3}\left(H_{n}\right)}{\digamma_{3}(\widetilde{H})}\right)\right] \\
& +\frac{m(1-\varepsilon) e^{-\mu_{1} \tau_{1}-\mu_{3} \tau_{3}}}{\alpha+m} \Lambda(\widetilde{F}, \widetilde{H})\left(\sum_{j=n-m_{1}+1}^{n} G\left(\frac{\Lambda\left(F_{j+1}, H_{j}\right)}{\Lambda(\widetilde{F}, \widetilde{H})}\right)\right. \\
& \left.-\sum_{j=n-m_{1}}^{n-1} G\left(\frac{\Lambda\left(F_{j+1}, H_{j}\right)}{\Lambda(\widetilde{F}, \widetilde{H})}\right)\right) \\
& +\varepsilon e^{-\mu_{2} \tau_{2}-\mu_{3} \tau_{3}} \Lambda(\widetilde{F}, \widetilde{H})\left(\sum_{j=n-m_{2}+1}^{n} G\left(\frac{\Lambda\left(F_{j+1}, H_{j}\right)}{\Lambda(\widetilde{F}, \widetilde{H})}\right)-\sum_{j=n-m_{2}}^{n-1} G\left(\frac{\Lambda\left(F_{j+1}, H_{j}\right)}{\Lambda(\widetilde{F}, \widetilde{H})}\right)\right) \\
& +\gamma \Lambda(\widetilde{F}, \widetilde{H})\left(\sum_{j=n-m_{3}+1}^{n} G\left(\frac{\digamma_{2}\left(S_{j+1}\right)}{\digamma_{2}(\widetilde{S})}\right)-\sum_{j=n-m_{3}}^{n-1} G\left(\frac{\digamma_{2}\left(S_{j+1}\right)}{\digamma_{2}(\widetilde{S})}\right)\right) .
\end{aligned}
$$

Using inequalities (45) and (46) by replacing $F^{*}, H^{*}, \rho^{*}$ with $\widetilde{F}, \widetilde{H}, \widetilde{\rho}$, we obtain

$$
\begin{aligned}
\Delta \mathcal{V}_{n} \leq & \frac{1}{\phi(h)}\left[\gamma\left(1-\frac{\Lambda(\widetilde{F}, \widetilde{H})}{\Lambda\left(\digamma_{n+1}, \widetilde{H}\right)}\right)\left(F_{n+1}-F_{n}\right)+\frac{m e^{-\mu_{3} \tau_{3}}}{\alpha+m}\left(1-\frac{\digamma_{1}(\widetilde{K})}{\digamma_{1}\left(K_{n+1}\right)}\right)\left(K_{n+1}-K_{n}\right)\right. \\
& +e^{-\mu_{3} \tau_{3}}\left(1-\frac{\digamma_{2}(\widetilde{S})}{\digamma_{2}\left(S_{n+1}\right)}\right)\left(S_{n+1}-S_{n}\right) \\
& +\frac{\left(a+\lambda \digamma_{5}(\widetilde{Z})\right)}{\theta}\left(1-\frac{\digamma_{3}(\widetilde{H})}{\digamma_{3}\left(H_{n+1}\right)}\right)\left(H_{n+1}-H_{n}\right) \\
& +\frac{d\left(a+\lambda \digamma_{5}(\widetilde{Z})\right)}{q \theta}\left(1-\frac{\digamma_{4}(\widetilde{Y})}{\digamma_{4}\left(Y_{n+1}\right)}\right)\left(Y_{n+1}-Y_{n}\right) \\
& \left.+\frac{\lambda e^{-\mu_{3} \tau_{3}}}{g}\left(1-\frac{\digamma_{5}(\widetilde{Z})}{\digamma_{5}\left(Z_{n+1}\right)}\right)\left(Z_{n+1}-Z_{n}\right)\right] \\
& +\frac{\left(c+d \digamma_{4}(\widetilde{Y})\right)}{\theta}\left(a+\lambda \digamma_{5}(\widetilde{Z})\right) \digamma_{3}(\widetilde{H})\left[\frac{\digamma_{3}\left(H_{n+1}\right)}{\digamma_{3}(\widetilde{H})}-\frac{\digamma_{3}\left(H_{n}\right)}{\digamma_{3}(\widetilde{H})}+\ln \left(\frac{\digamma_{3}\left(H_{n}\right)}{\digamma_{3}\left(H_{n+1}\right)}\right)\right]
\end{aligned}
$$




$$
\begin{aligned}
& +\frac{m(1-\varepsilon) e^{-\mu_{1} \tau_{1}-\mu_{3} \tau_{3}}}{\alpha+m} \Lambda(\widetilde{F}, \widetilde{H})\left[\frac{\Lambda\left(F_{n+1}, H_{n}\right)}{\Lambda(\widetilde{F}, \widetilde{H})}-\frac{\Lambda\left(F_{n-m_{1}+1}, H_{n-m_{1}}\right)}{\Lambda(\widetilde{F}, \widetilde{H})}\right. \\
& \left.+\ln \left(\frac{\Lambda\left(F_{n-m_{1}+1}, H_{n-m_{1}}\right)}{\Lambda\left(F_{n+1}, H_{n}\right)}\right)\right] \\
& +\varepsilon e^{-\mu_{2} \tau_{2}-\mu_{3} \tau_{3}} \Lambda(\widetilde{F}, \widetilde{H})\left[\frac{\Lambda\left(F_{n+1}, H_{n}\right)}{\Lambda(\widetilde{F}, \widetilde{H})}-\frac{\Lambda\left(F_{n-m_{2}+1}, H_{n-m_{2}}\right)}{\Lambda(\widetilde{F}, \widetilde{H})}\right. \\
& \left.+\ln \left(\frac{\Lambda\left(F_{n-m_{2}+1}, H_{n-m_{2}}\right)}{\Lambda\left(F_{n+1}, H_{n}\right)}\right)\right] \\
& +\gamma \Lambda(\widetilde{F}, \widetilde{H})\left[\frac{\digamma_{2}\left(S_{n+1}\right)}{\digamma_{2}(\widetilde{S})}-\frac{\digamma_{2}\left(S_{n-m_{3}+1}\right)}{\digamma_{2}(\widetilde{S})}+\ln \left(\frac{\digamma_{2}\left(S_{n-m_{3}+1}\right)}{\digamma_{2}\left(S_{n+1}\right)}\right)\right] .
\end{aligned}
$$

From Eqs. (11)-(16) we have

$$
\begin{aligned}
& \Delta \mathcal{V}_{n} \leq \gamma\left(1-\frac{\Lambda(\widetilde{F}, \widetilde{H})}{\Lambda\left(F_{n+1}, \widetilde{H}\right)}\right)\left(\Theta\left(F_{n+1}\right)-\Lambda\left(F_{n+1}, H_{n}\right)\right) \\
& +\frac{m e^{-\mu_{3} \tau_{3}}}{\alpha+m}\left(1-\frac{\digamma_{1}(\widetilde{K})}{\digamma_{1}\left(K_{n+1}\right)}\right)\left[(1-\varepsilon) e^{-\mu_{1} \tau_{1}} \Lambda\left(F_{n-m_{1}+1}, H_{n-m_{1}}\right)\right. \\
& \left.-(\alpha+m) \digamma_{1}\left(K_{n+1}\right)\right] \\
& +e^{-\mu_{3} \tau_{3}}\left(1-\frac{\digamma_{2}(\widetilde{S})}{\digamma_{2}\left(S_{n+1}\right)}\right)\left[\varepsilon e^{-\mu_{2} \tau_{2}} \Lambda\left(F_{n-m_{2}+1}, H_{n-m_{2}}\right)+m \digamma_{1}\left(K_{n+1}\right)-a \digamma_{2}\left(S_{n+1}\right)\right. \\
& \left.-\lambda \digamma_{2}\left(S_{n+1}\right) \digamma_{5}\left(Z_{n+1}\right)\right] \\
& +\frac{\left(a+\lambda \digamma_{5}(\widetilde{Z})\right)}{\theta}\left(1-\frac{\digamma_{3}(\widetilde{H})}{\digamma_{3}\left(H_{n+1}\right)}\right)\left[\theta e^{-\mu_{3} \tau_{3}} \digamma_{2}\left(S_{n-m_{3}+1}\right)-c \digamma_{3}\left(H_{n+1}\right)\right. \\
& \left.-d \digamma_{3}\left(H_{n+1}\right) \digamma_{4}\left(Y_{n+1}\right)\right] \\
& +\frac{d\left(a+\lambda \digamma_{5}(\widetilde{Z})\right)}{q \theta}\left(1-\frac{\digamma_{4}(\widetilde{Y})}{\digamma_{4}\left(Y_{n+1}\right)}\right)\left[q \digamma_{3}\left(H_{n+1}\right) \digamma_{4}\left(Y_{n+1}\right)-\eta \digamma_{4}\left(Y_{n+1}\right)\right] \\
& +\frac{\lambda e^{-\mu_{3} \tau_{3}}}{g}\left(1-\frac{\digamma_{5}(\widetilde{Z})}{\digamma_{5}\left(Z_{n+1}\right)}\right)\left[g \digamma_{2}\left(S_{n+1}\right) \digamma_{5}\left(Z_{n+1}\right)-\xi \digamma_{5}\left(Z_{n+1}\right)\right] \\
& +\frac{\left(c+d \digamma_{4}(\widetilde{Y})\right)}{\theta}\left(a+\lambda \digamma_{5}(\widetilde{Z})\right) \digamma_{3}(\widetilde{H})\left[\frac{\digamma_{3}\left(H_{n+1}\right)}{\digamma_{3}(\widetilde{H})}-\frac{\digamma_{3}\left(H_{n}\right)}{\digamma_{3}(\widetilde{H})}+\ln \left(\frac{\digamma_{3}\left(H_{n}\right)}{\digamma_{3}\left(H_{n+1}\right)}\right)\right] \\
& +\frac{m(1-\varepsilon) e^{-\mu_{1} \tau_{1}-\mu_{3} \tau_{3}}}{\alpha+m} \Lambda(\widetilde{F}, \widetilde{H})\left[\frac{\Lambda\left(F_{n+1}, H_{n}\right)}{\Lambda(\widetilde{F}, \widetilde{H})}-\frac{\Lambda\left(F_{n-m_{1}+1}, H_{n-m_{1}}\right)}{\Lambda(\widetilde{F}, \widetilde{H})}\right. \\
& \left.+\ln \left(\frac{\Lambda\left(F_{n-m_{1}+1}, H_{n-m_{1}}\right)}{\Lambda\left(F_{n+1}, H_{n}\right)}\right)\right] \\
& +\varepsilon e^{-\mu_{2} \tau_{2}-\mu_{3} \tau_{3}} \Lambda(\widetilde{F}, \widetilde{H})\left[\frac{\Lambda\left(F_{n+1}, H_{n}\right)}{\Lambda(\widetilde{F}, \widetilde{H})}-\frac{\Lambda\left(F_{n-m_{2}+1}, H_{n-m_{2}}\right)}{\Lambda(\widetilde{F}, \widetilde{H})}\right. \\
& \left.+\ln \left(\frac{\Lambda\left(F_{n-m_{2}+1}, H_{n-m_{2}}\right)}{\Lambda\left(F_{n+1}, H_{n}\right)}\right)\right] \\
& +\gamma \Lambda(\widetilde{F}, \widetilde{H})\left[\frac{\digamma_{2}\left(S_{n+1}\right)}{\digamma_{2}(\widetilde{S})}-\frac{\digamma_{2}\left(S_{n-m_{3}+1}\right)}{\digamma_{2}(\widetilde{S})}+\ln \left(\frac{\digamma_{2}\left(S_{n-m_{3}+1}\right)}{\digamma_{2}\left(S_{n+1}\right)}\right)\right], \\
& \Delta \mathcal{V}_{n} \leq \gamma\left(1-\frac{\Lambda(\widetilde{F}, \widetilde{H})}{\Lambda\left(F_{n+1}, \widetilde{H}\right)}\right)\left(\Theta\left(F_{n+1}\right)-\Theta(\widetilde{F})\right)+\gamma \Theta(\widetilde{F})\left(1-\frac{\Lambda(\widetilde{F}, \widetilde{H})}{\Lambda\left(F_{n+1}, \widetilde{H}\right)}\right)
\end{aligned}
$$




$$
\begin{aligned}
& +\gamma \frac{\Lambda(\widetilde{F}, \widetilde{H})}{\Lambda\left(F_{n+1}, \widetilde{H}\right)} \Lambda\left(F_{n+1}, H_{n}\right)-\frac{m(1-\varepsilon) e^{-\mu_{1} \tau_{1}-\mu_{3} \tau_{3}}}{\alpha+m} \Lambda\left(F_{n-m_{1}+1}, H_{n-m_{1}}\right) \frac{\digamma_{1}(\widetilde{K})}{\digamma_{1}\left(K_{n+1}\right)} \\
& +m e^{-\mu_{3} \tau_{3}} \digamma_{1}(\widetilde{K})-\varepsilon e^{-\mu_{2} \tau_{2}-\mu_{3} \tau_{3}} \Lambda\left(F_{n-m_{2}+1}, H_{n-m_{2}}\right) \frac{\digamma_{2}(\widetilde{S})}{\digamma_{2}\left(S_{n+1}\right)} \\
& -m e^{-\mu_{3} \tau_{3}} \digamma_{1}\left(K_{n+1}\right) \frac{\digamma_{2}(\widetilde{S})}{\digamma_{2}\left(S_{n+1}\right)} \\
& -a e^{-\mu_{3} \tau_{3}} \digamma_{2}\left(S_{n+1}\right)+\lambda e^{-\mu_{3} \tau_{3}} \digamma_{2}(\widetilde{S}) \digamma_{5}\left(Z_{n+1}\right)+a e^{-\mu_{3} \tau_{3}} \digamma_{2}(\widetilde{S}) \\
& +\left(a+\lambda \digamma_{5}(\widetilde{Z})\right) e^{-\mu_{3} \tau_{3}} \digamma_{2}\left(S_{n-m_{3}+1}\right) \\
& -\left(a+\lambda \digamma_{5}(\widetilde{Z})\right) e^{-\mu_{3} \tau_{3}} \digamma_{2}\left(S_{n-m_{3}+1}\right) \frac{\digamma_{3}(\widetilde{H})}{\digamma_{3}\left(H_{n+1}\right)}+\frac{\left(a+\lambda \digamma_{5}(\widetilde{Z})\right) c}{\theta} \digamma_{3}(\widetilde{H}) \\
& +\frac{d\left(a+\lambda \digamma_{5}(\widetilde{Z})\right)}{q \theta} \digamma_{3}(\widetilde{H}) \digamma_{4}\left(Y_{n+1}\right)-\frac{d\left(a+\lambda \digamma_{5}(\widetilde{Z})\right) \eta}{q \theta} \digamma_{4}\left(Y_{n+1}\right) \\
& +\frac{d\left(a+\lambda \digamma_{5}(\widetilde{Z})\right) \eta}{q \theta} \digamma_{4}(\widetilde{Y})-\lambda e^{-\mu_{3} \tau_{3}} \digamma_{5}(\widetilde{Z}) \digamma_{2}\left(S_{n+1}\right)-\frac{\lambda e^{-\mu_{3} \tau_{3}} \xi}{g} \digamma_{5}\left(Z_{n+1}\right) \\
& +\frac{\lambda e^{-\mu_{3} \tau_{3} \xi}}{g} \digamma_{5}(\widetilde{Z}) \\
& -\frac{\left(c+d \digamma_{4}(\tilde{Y})\right)}{\theta}\left(a+\lambda \digamma_{5}(\widetilde{Z})\right) \digamma_{3}\left(H_{n}\right) \\
& +\frac{\left(c+d \digamma_{4}(\tilde{Y})\right)}{\theta}\left(a+\lambda \digamma_{5}(\widetilde{Z})\right) \digamma_{3}(\widetilde{H}) \ln \left(\frac{\digamma_{3}\left(H_{n}\right)}{\digamma_{3}\left(H_{n+1}\right)}\right) \\
& +\frac{m(1-\varepsilon) e^{-\mu_{1} \tau_{1}-\mu_{3} \tau_{3}}}{\alpha+m} \Lambda(\widetilde{F}, \widetilde{H}) \ln \left(\frac{\Lambda\left(F_{n-m_{1}+1}, H_{n-m_{1}}\right)}{\Lambda\left(F_{n+1}, H_{n}\right)}\right) \\
& +\varepsilon e^{-\mu_{2} \tau_{2}-\mu_{3} \tau_{3}} \Lambda(\widetilde{F}, \tilde{H}) \ln \left(\frac{\Lambda\left(F_{n-m_{2}+1}, H_{n-m_{2}}\right)}{\Lambda\left(F_{n+1}, H_{n}\right)}\right) \\
& +\gamma \Lambda(\widetilde{F}, \widetilde{H})\left(\frac{\digamma_{2}\left(S_{n+1}\right)}{\digamma_{2}(\widetilde{S})}-\frac{\digamma_{2}\left(S_{n-m_{3}+1}\right)}{\digamma_{2}(\widetilde{S})}+\ln \left(\frac{\digamma_{2}\left(S_{n-m_{3}+1}\right)}{\digamma_{2}\left(S_{n+1}\right)}\right)\right) \text {. }
\end{aligned}
$$

Using the conditions of $\widetilde{Q}$

$$
\begin{aligned}
& \Theta(\widetilde{F})=\Lambda(\widetilde{F}, \widetilde{H}), \\
& (1-\varepsilon) e^{-\mu_{1} \tau_{1}} \Lambda(\widetilde{F}, \widetilde{H})=(\alpha+m) \digamma_{1}(\widetilde{K}), \\
& \varepsilon e^{-\mu_{2} \tau_{2}-\mu_{3} \tau_{3}} \Lambda(\widetilde{F}, \widetilde{H})+m e^{-\mu_{3} \tau_{3}} \digamma_{1}(\widetilde{K})=\gamma \Lambda(\widetilde{F}, \widetilde{H})=\left(a+\lambda \digamma_{5}(\widetilde{Z})\right) e^{-\mu_{3} \tau_{3}} \digamma_{2}(\widetilde{S}), \\
& \theta e^{-\mu_{3} \tau_{3}} \digamma_{2}(\widetilde{S})=\left(c+d \digamma_{4}(\widetilde{Y})\right) \digamma_{3}(\widetilde{H}), \\
& \digamma_{2}(\widetilde{S})=\frac{\xi}{g}, \quad \digamma_{3}(\widetilde{H})=\frac{\eta}{q},
\end{aligned}
$$

we get

$$
\begin{aligned}
\Delta \mathcal{V}_{n} \leq & \gamma\left(1-\frac{\Lambda(\widetilde{F}, \widetilde{H})}{\Lambda\left(F_{n+1}, \widetilde{H}\right)}\right)\left(\Theta\left(F_{n+1}\right)-\Theta(\widetilde{F})\right)+\gamma \Lambda(\widetilde{F}, \widetilde{H})\left(1-\frac{\Lambda(\widetilde{F}, \widetilde{H})}{\Lambda\left(F_{n+1}, \widetilde{H}\right)}\right) \\
& +\gamma \Lambda(\widetilde{F}, \widetilde{H}) \frac{\Lambda\left(F_{n+1}, H_{n}\right)}{\Lambda\left(F_{n+1}, \widetilde{H}\right)}
\end{aligned}
$$




$$
\begin{aligned}
& -\frac{m(1-\varepsilon) e^{-\mu_{1} \tau_{1}-\mu_{3} \tau_{3}}}{\alpha+m} \Lambda(\widetilde{F}, \widetilde{H}) \frac{\Lambda\left(F_{n-m_{1}+1}, H_{n-m_{1}}\right) \digamma_{1}(\widetilde{K})}{\Lambda(\widetilde{F}, \widetilde{H}) \digamma_{1}\left(K_{n+1}\right)} \\
& +\frac{m(1-\varepsilon) e^{-\mu_{1} \tau_{1}-\mu_{3} \tau_{3}}}{\alpha+m} \Lambda(\widetilde{F}, \widetilde{H}) \\
& -\varepsilon e^{-\mu_{2} \tau_{2}-\mu_{3} \tau_{3}} \Lambda(\widetilde{F}, \widetilde{H}) \frac{\Lambda\left(F_{n-m_{2}+1}, H_{n-m_{2}}\right) \digamma_{2}(\widetilde{S})}{\Lambda(\widetilde{F}, \widetilde{H}) \digamma_{2}\left(S_{n+1}\right)} \\
& -\frac{m(1-\varepsilon) e^{-\mu_{1} \tau_{1}-\mu_{3} \tau_{3}}}{\alpha+m} \Lambda(\widetilde{F}, \widetilde{H}) \frac{\digamma_{1}\left(K_{n+1}\right) \digamma_{2}(\widetilde{S})}{\digamma_{1}(\widetilde{K}) \digamma_{2}\left(S_{n+1}\right)}+\gamma \Lambda(\widetilde{F}, \widetilde{H}) \\
& -\gamma \Lambda(\widetilde{F}, \widetilde{H}) \frac{\digamma_{3}(\widetilde{H}) \digamma_{2}\left(S_{n-m_{3}+1}\right)}{\digamma_{3}\left(H_{n+1}\right) \digamma_{2}(\widetilde{S})} \\
& +\gamma \Lambda(\widetilde{F}, \widetilde{H})-\gamma \Lambda(\widetilde{F}, \widetilde{H}) \frac{\digamma_{3}\left(H_{n}\right)}{\digamma_{3}(\widetilde{H})} \\
& +\gamma \Lambda(\widetilde{F}, \widetilde{H}) \ln \left(\frac{\digamma_{3}\left(H_{n}\right)}{\digamma_{3}\left(H_{n+1}\right)}\right) \\
& +\frac{m(1-\varepsilon) e^{-\mu_{1} \tau_{1}-\mu_{3} \tau_{3}}}{\alpha+m} \Lambda(\widetilde{F}, \widetilde{H}) \ln \left(\frac{\Lambda\left(F_{n-m_{1}+1}, H_{n-m_{1}}\right)}{\Lambda\left(F_{n+1}, H_{n}\right)}\right) \\
& +\varepsilon e^{-\mu_{2} \tau_{2}-\mu_{3} \tau_{3}} \Lambda(\widetilde{F}, \tilde{H}) \ln \left(\frac{\Lambda\left(F_{n-m_{2}+1}, H_{n-m_{2}}\right)}{\Lambda\left(F_{n+1}, H_{n}\right)}\right) \\
& +\gamma \Lambda(\widetilde{F}, \tilde{H}) \ln \left(\frac{\digamma_{2}\left(S_{n-m_{3}+1}\right)}{\digamma_{2}\left(S_{n+1}\right)}\right) \\
& =\gamma\left(1-\frac{\Lambda(\widetilde{F}, \widetilde{H})}{\Lambda\left(F_{n+1}, \widetilde{H}\right)}\right)\left(\Theta\left(F_{n+1}\right)-\Theta(\widetilde{F})\right) \\
& +\frac{m(1-\varepsilon) e^{-\mu_{1} \tau_{1}-\mu_{3} \tau_{3}}}{\alpha+m} \Lambda(\widetilde{F}, \widetilde{H})\left[5-\frac{\Lambda(\widetilde{F}, \widetilde{H})}{\Lambda\left(F_{n+1}, \widetilde{H}\right)}\right. \\
& -\frac{\Lambda\left(F_{n-m_{1}+1}, H_{n-m_{1}}\right) \digamma_{1}(\widetilde{K})}{\Lambda(\widetilde{F}, \widetilde{H}) \digamma_{1}\left(K_{n+1}\right)}-\frac{\digamma_{1}\left(K_{n+1}\right) \digamma_{2}(\widetilde{S})}{\digamma_{1}(\widetilde{K}) \digamma_{2}\left(S_{n+1}\right)} \\
& -\frac{\digamma_{3}(\widetilde{H}) \digamma_{2}\left(S_{n-m_{3}+1}\right)}{\digamma_{3}\left(H_{n+1}\right) \digamma_{2}(\widetilde{S})}-\frac{\Lambda\left(F_{n+1}, \widetilde{H}\right) \digamma_{3}\left(H_{n}\right)}{\Lambda\left(F_{n+1}, H_{n}\right) \digamma_{3}(\widetilde{H})} \\
& \left.+\ln \left(\frac{\Lambda\left(F_{n-m_{1}+1}, H_{n-m_{1}}\right) \digamma_{2}\left(S_{n-m_{3}+1}\right) \digamma_{3}\left(H_{n}\right)}{\Lambda\left(F_{n+1}, H_{n}\right) \digamma_{2}\left(S_{n+1}\right) \digamma_{3}\left(H_{n+1}\right)}\right)\right] \\
& +\varepsilon e^{-\mu_{2} \tau_{2}-\mu_{3} \tau_{3}} \Lambda(\widetilde{F}, \widetilde{H})\left[4-\frac{\Lambda(\widetilde{F}, \widetilde{H})}{\Lambda\left(F_{n+1}, \widetilde{H}\right)}-\frac{\Lambda\left(F_{n-m_{2}+1}, H_{n-m_{2}}\right) \digamma_{2}(\widetilde{S})}{\Lambda(\widetilde{F}, \widetilde{H}) \digamma_{2}\left(S_{n+1}\right)}\right. \\
& -\frac{\digamma_{3}(\widetilde{H}) \digamma_{2}\left(S_{n-m_{3}+1}\right)}{\digamma_{3}\left(H_{n+1}\right) \digamma_{2}(\widetilde{S})} \\
& \left.-\frac{\Lambda\left(F_{n+1}, \widetilde{H}\right) \digamma_{3}\left(H_{n}\right)}{\Lambda\left(F_{n+1}, H_{n}\right) \digamma_{3}(\widetilde{H})}+\ln \left(\frac{\Lambda\left(F_{n-m_{2}+1}, H_{n-m_{2}}\right) \digamma_{2}\left(S_{n-m_{3}+1}\right) \digamma_{3}\left(H_{n}\right)}{\Lambda\left(F_{n+1}, H_{n}\right) \digamma_{2}\left(S_{n+1}\right) \digamma_{3}\left(H_{n+1}\right)}\right)\right] \\
& +\gamma \Lambda(\widetilde{F}, \widetilde{H})\left[-1+\frac{\Lambda\left(F_{n+1}, \widetilde{H}\right) \digamma_{3}\left(H_{n}\right)}{\Lambda\left(F_{n+1}, H_{n}\right) \digamma_{3}(\widetilde{H})}+\frac{\Lambda\left(F_{n+1}, H_{n}\right)}{\Lambda\left(F_{n+1}, \widetilde{H}\right)}-\frac{\digamma_{3}\left(H_{n}\right)}{\digamma_{3}(\widetilde{H})}\right], \\
& \Delta \mathcal{V}_{n} \leq \gamma\left(1-\frac{\Lambda(\widetilde{F}, \widetilde{H})}{\Lambda\left(F_{n+1}, \widetilde{H}\right)}\right)\left(\Theta\left(F_{n+1}\right)-\Theta(\widetilde{F})\right) \\
& -\frac{m(1-\varepsilon) e^{-\mu_{1} \tau_{1}-\mu_{3} \tau_{3}}}{\alpha+m} \Lambda(\widetilde{F}, \widetilde{H})\left[G\left(\frac{\Lambda(\widetilde{F}, \widetilde{H})}{\Lambda\left(F_{n+1}, \widetilde{H}\right)}\right)\right.
\end{aligned}
$$




$$
\begin{aligned}
& +G\left(\frac{\Lambda\left(F_{n-m_{1}+1}, H_{n-m_{1}}\right) \digamma_{1}(\widetilde{K})}{\Lambda(\widetilde{F}, \widetilde{H}) \digamma_{1}\left(K_{n+1}\right)}\right)+G\left(\frac{\digamma_{1}\left(K_{n+1}\right) \digamma_{2}(\widetilde{S})}{\digamma_{1}(\widetilde{K}) \digamma_{2}\left(S_{n+1}\right)}\right) \\
& +G\left(\frac{\digamma_{3}(\widetilde{H}) \digamma_{2}\left(S_{n-m_{3}+1}\right)}{\digamma_{3}\left(H_{n+1}\right) \digamma_{2}(\widetilde{S})}\right) \\
& \left.+G\left(\frac{\Lambda\left(F_{n+1}, \widetilde{H}\right) \digamma_{3}\left(H_{n}\right)}{\Lambda\left(F_{n+1}, H_{n}\right) \digamma_{3}(\widetilde{H})}\right)\right]-\varepsilon e^{-\mu_{2} \tau_{2}-\mu_{3} \tau_{3}} \Lambda(\widetilde{F}, \widetilde{H})\left[G\left(\frac{\Lambda(\widetilde{F}, \widetilde{H})}{\Lambda\left(F_{n+1}, \widetilde{H}\right)}\right)\right. \\
& +G\left(\frac{\Lambda\left(F_{n-m_{2}+1}, H_{n-m_{2}}\right) \digamma_{2}(\widetilde{S})}{\Lambda(\widetilde{F}, \widetilde{H}) \digamma_{2}\left(S_{n+1}\right)}\right)+G\left(\frac{\digamma_{3}(\widetilde{H}) \digamma_{2}\left(S_{n-m_{3}+1}\right)}{\digamma_{3}\left(H_{n+1}\right) \digamma_{2}(\widetilde{S})}\right) \\
& \left.+G\left(\frac{\Lambda\left(F_{n+1}, \widetilde{H}\right) \digamma_{3}\left(H_{n}\right)}{\Lambda\left(F_{n+1}, H_{n}\right) \digamma_{3}(\widetilde{H})}\right)\right] \\
& +\gamma \Lambda(\widetilde{F}, \widetilde{H})\left(1-\frac{\Lambda\left(F_{n+1}, \widetilde{H}\right)}{\Lambda\left(F_{n+1}, H_{n}\right)}\right)\left(\frac{\Lambda\left(F_{n+1}, H_{n}\right)}{\Lambda\left(F_{n+1}, \widetilde{H}\right)}-\frac{\digamma_{3}\left(H_{n}\right)}{\digamma_{3}(\widetilde{H})}\right) .
\end{aligned}
$$

Using Conditions C1-C4, we get that the first and last terms of Eq. (59) are less than or equal to zero. Thus, $\mathcal{V}_{n}$ is a monotone decreasing sequence. Since $\mathcal{V}_{n} \geq 0$, then there is a limit $\lim _{n \rightarrow \infty} \mathcal{V}_{n} \geq 0$. Therefore, $\lim _{n \rightarrow \infty} \Delta \mathcal{V}_{n}=0$, which implies that $\lim _{n \rightarrow \infty} F_{n}=\widetilde{F}$, $\lim _{n \rightarrow \infty} K_{n}=\widetilde{K}, \lim _{n \rightarrow \infty} S_{n}=\widetilde{S}, \lim _{n \rightarrow \infty} H_{n}=\widetilde{H}$. From Eqs. (13) and (14) we have

$$
\begin{aligned}
& 0=\varepsilon e^{-\mu_{2} \tau_{2}} \Lambda(\widetilde{F}, \widetilde{H})+m \digamma_{1}(\widetilde{K})-a \digamma_{2}(\widetilde{S})-\lambda \digamma_{2}(\widetilde{S}) \lim _{n \rightarrow \infty} \digamma_{5}\left(Z_{n+1}\right), \\
& 0=\theta e^{-\mu_{3} \tau_{3}} \digamma_{2}(\widetilde{S})-c \digamma_{3}(\widetilde{H})-d \digamma_{2}(\widetilde{H}) \lim _{n \rightarrow \infty} \digamma_{4}\left(Y_{n+1}\right),
\end{aligned}
$$

then $\lim _{n \rightarrow \infty} Y_{n}=\widetilde{Y}$ and $\lim _{n \rightarrow \infty} Z_{n}=\widetilde{Z}$. Then we get $\widetilde{Q}$ is G.A.S.

\section{Acknowledgements}

This project was funded by the Deanship of Scientific Research (DSR), King Abdulaziz University, Jeddah, under grant No. (D-434-130-1441). The authors, therefore, gratefully acknowledge the DSR technical and financial support.

Funding

Not applicable.

\section{Availability of data and materials}

Not applicable.

\section{Competing interests}

The authors declare that they have no competing interests.

\section{Authors' contributions}

All authors contributed equally to the writing of this paper. All authors read and approved the final manuscript.

\section{Author details}

'Department of Mathematics, Faculty of Science, King Abdulaziz University, Jeddah, Saudi Arabia. ${ }^{2}$ Department of Mathematics, Faculty of Science, Taif University, Taif, Saudi Arabia.

\section{Publisher's Note}

Springer Nature remains neutral with regard to jurisdictional claims in published maps and institutional affiliations.

Received: 4 December 2019 Accepted: 20 January 2020 Published online: 03 February 2020

\section{References}

1. Nowak, M.A., Bangham, C.R.M.: Population dynamics of immune responses to persistent viruses. Science $272,74-79$ (1996)

2. Perelson, A.S. Nelson, P.W: Mathematical analysis of HIV-1 dynamics in vivo. SIAM Rev. 41, 3-44 (1999)

3. Callaway, D.S., Perelson, A.S.: HIV-1 infection and low steady state viral loads. Bull. Math. Biol. 64, 29-64 (2002)

4. Neumann, A.U., Lam, N.P., Dahari, H., Gretch, D.R., Wiley, T.E., Layden, T.J., Perelson, A.S.: Hepatitis C viral dynamics in vivo and the antiviral efficacy of interferon-alpha therapy. Science 282, 103-107 (1998) 
5. Zhang, $\mathrm{S} ., \mathrm{Xu}, \mathrm{X}$ : Dynamic analysis and optimal control for a model of hepatitis $\mathrm{C}$ with treatment. Commun. Nonlinear Sci. Numer. Simul. 46, 14-25 (2017)

6. Wang, K., Fan, A., Torres, A.: Global properties of an improved hepatitis B virus model. Nonlinear Anal., Real World Appl. 11,3131-3138 (2010)

7. Yousfi, N., Hattaf, K., Tridane, A.: Modeling the adaptive immune response in HBV infection. J. Math. Biol. 63, 933-957 (2011)

8. Elaiw, A.M., Almalki, S.E., Hobiny, A.D.: Global properties of saturated chikungunya virus dynamics models with cellular infection and delays. Adv. Differ. Equ. 2019, 476 (2019)

9. Perera, S., Perera, S.S.N.: Mathematical modeling and analysis of innate and humoral immune responses to dengue infections. Int. J. Biomath. 12(7), Article ID 1950077 (2019)

10. Li, M.Y., Shu, H.: Global dynamics of a mathematical model for HTLV-l infection of CD4+ T cells with delayed CTL response. Nonlinear Anal., Real World Appl. 13, 1080-1092 (2012)

11. Shi, X., Zhou, X., Song, X.: Dynamical behavior of a delay virus dynamics model with CTL immune response. Nonlinear Anal., Real World Appl. 11, 1795-1809 (2010)

12. Pang, J., Cui, J.: Analysis of a hepatitis B viral infection model with immune response delay. Int. J. Biomath. 10(2), Article ID 1750020 (2017)

13. Shu, H., Wang, L., Watmough, J.: Global stability of a nonlinear viral infection model with infinitely distributed intracellular delays and CTL immune responses. SIAM J. Appl. Math. 73(3), 1280-1302 (2013)

14. Murase, A., Sasaki, T., Kajiwara, T.: Stability analysis of pathogen-immune interaction dynamics. J. Math. Biol. 51, 247-267 (2005)

15. Elaiw, A.M., AlShamrani, N.H.: Global stability of humoral immunity virus dynamics models with nonlinear infection rate and removal. Nonlinear Anal., Real World Appl. 26, 161-190 (2015)

16. Wang, S., Zou, D.: Global stability of in host viral models with humoral immunity and intracellular delays. Appl. Math. Model. 36, 1313-1322 (2012)

17. $\mathrm{Xu}$, J., Zhou, Y., Li, Y., Yang, Y.: Global dynamics of a intracellular infection model with delays and humoral immunity. Math. Methods Appl. Sci. 39(18), 5427-5435 (2016)

18. Perelson, A.S., Neumann, A.U., Markowitz, M., Leonard, J.M., Ho, D.D.: HIV-1 dynamics in vivo: virion clearance rate, infected cell life-span, and viral generation time. Science 271, 1582-1586 (1996)

19. Herz, A., Bonhoeffer, S., Anderson, R.M., May, R.M., Nowak, M.A.: Viral dynamics in vivo: limitations on estimates of intracellular delay and virus decay. Proc. Natl. Acad. Sci. 93, 7247-7251 (1996)

20. Elaiw, A.M., Elnahary, E.K., Raezah, A.A.: Effect of cellular reservoirs and delays on the global dynamics of HIV. Adv. Differ. Equ. 2018, 85 (2018)

21. Elaiw, A.M., Raezah, A.A., Azoz, S.A.: Stability of delayed HIV dynamics models with two latent reservoirs and immune impairment. Adv. Differ. Equ. 2018, 414 (2018)

22. Elaiw, A.M., AlShamrani, N.H.: Stability of a general delay-distributed virus dynamics model with multi-staged infected progression and immune response. Math. Methods Appl. Sci. 40(3), 699-719 (2017)

23. Elaiw, A.M., Raezah, A.A.: Stability of general virus dynamics models with both cellular and viral infections and delays. Math. Methods Appl. Sci. 40(16), 5863-5880 (2017)

24. Nelson, P.W., Murray, J.D., Perelson, A.S.: A model of HIV-1 pathogenesis that includes an intracellular delay. Math. Biosci. 163, 201-215 (2000)

25. Elaiw, A.M., AlShamrani, N.H.: Stability of a general adaptive immunity virus dynamics model with multi-stages of infected cells and two routes of infection. Math. Methods Appl. Sci. (2019). https://doi.org/10.1002/mma.5923

26. Elaiw, A.M., Almuallem, N.A.: Global properties of delayed-HIV dynamics models with differential drug efficacy in cocirculating target cells. Appl. Math. Comput. 265, 1067-1089 (2015)

27. Elaiw, A.M., Almuallem, N.A.: Global dynamics of delay-distributed HIV infection models with differential drug efficacy in cocirculating target cells. Math. Methods Appl. Sci. 39, 4-31 (2016)

28. Xu, R., Ma, Z:: An HBV model with diffusion and time delay. J. Theor. Biol. 257, 499-509 (2009)

29. Zhang, Y., Xu, Z.: Dynamics of a diffusive HBV model with delayed Beddington-DeAngelis response. Nonlinear Anal., Real World Appl. 15, 118-139 (2014)

30. Elaiw, A.M., Elnahary, E.K.: Analysis of general humoral immunity HIV dynamics model with HAART and distributed delays. Mathematics 7(2), Article ID 157 (2019)

31. Elaiw, A.M., Almatrafi, A., Hobiny, A.D., Hattaf, K.: Global properties of a general latent pathogen dynamics model with delayed pathogenic and cellular infections. Discrete Dyn. Nat. Soc. 2019, Article ID 9585497 (2019)

32. Zhao, Y., Xu, Z.: Global dynamics for a delayed hepatitis C virus, infection model. Electron. J. Differ. Equ. 2014(132), 1 (2014)

33. Wodarz, D.: Hepatitis C virus dynamics and pathology: the role of CTL and antibody responses. J. Gen. Virol. 84, 1743-1750 (2003)

34. Wang, J., Pang, J., Kuniya, T., Enatsu, Y.: Global threshold dynamics in a five-dimensional virus model with cell-mediated, humoral immune responses and distributed delays. Appl. Math. Comput. 241(15), 298-316 (2014)

35. Yan, Y., Wang, W.: Global stability of a five-dimensional model with immune responses and delay. Discrete Contin. Dyn. Syst., Ser. B 17, 401-416 (2012)

36. Wang, X., Liu, S.: A class of delayed viral models with saturation infection rate and immune response. Math. Methods Appl. Sci. 36, 125-142 (2013)

37. Chun, T.-W., Stuyver, L., Mizell, S.B., Ehler, L.A., Mican, J.A.M., Baseler, M., Lloyd, A.L., Nowak, M.A., Fauci, A.S.: Presence of an inducible HIV-1 latent reservoir during highly active antiretroviral therapy. Proc. Natl. Acad. Sci. 94, 13193-13197 (1997)

38. Wong, J.K., Hezareh, M., Gunthard, H.F., Havlir, D.V., Ignacio, C.C., Spina, C.A., Richman, D.D.: Recovery of replication-competent HIV despite prolonged suppression of plasma viremia. Science 278, 1291-1295 (1997)

39. Elaiw, A.M., AlShamrani, N.H.: Stability of an adaptive immunity pathogen dynamics model with latency and multiple delays. Math. Methods Appl. Sci. 36, 125-142 (2018)

40. Korobeinikov, A.: Global asymptotic properties of virus dynamics models with dose-dependent parasite reproduction and virulence and non-linear incidence rate. Math. Med. Biol. 26(3), 225-239 (2009). https://doi.org/10.1093/imammb/dqp006 
41. Mickens, R.E.: Nonstandard Finite Difference Models of Differential Equations. World Scientific, Singapore (1994)

42. Korpusik, A.: A nonstandard finite difference scheme for a basic model of cellular immune response to viral infection. Commun. Nonlinear Sci. Numer. Simul. 43, 369-384 (2017)

43. Xu, J., Geng, Y., Hou, J.: A nonstandard finite difference scheme for a delayed and diffusive viral infection model with general nonlinear incidence rate. Comput. Math. Appl. 74, 1782-1798 (2017)

44. Yang, Y., Xinsheng, M., Yahui, L.: Global stability of a discrete virus dynamics model with Holling type-ll infection function. Math. Methods Appl. Sci. 39, 2078-2082 (2016)

45. Geng, Y., Xu, J., Hou, J.: Discretization and dynamic consistency of a delayed and diffusive viral infection model. Appl. Math. Comput. 316, 282-295 (2018)

46. Xu, J., Hou, J., Geng, Y., Zhang, S.: Dynamic consistent NSFD scheme for a viral infection model with cellular infection and general nonlinear incidence. Adv. Differ. Equ. 2018, 108 (2018)

47. Wang, J., Teng, Z., Miao, H.: Global dynamics for discrete-time analog of viral infection model with nonlinear incidence and CTL immune response. Adv. Differ. Equ. 2016, Article ID 143 (2016). https://doi.org/10.1186/s13662-016-0862-y

48. Xu, J., Geng, Y.: Stabilty preserving NSFD scheme for a delayed viral infection model with cell-to-cell transmission and general nonlinear incidence. J. Differ. Equ. Appl. 23(5), 893-916 (2017)

49. Elaiw, A.M., Alshaikh, M.A.: Stability analysis of a general discrete-time pathogen infection model with humoral immunity. J. Differ. Equ. Appl. 25(8), 1149-1172 (2019)

50. Elaiw, A.M., Alshaikh, M.A.: Stability of discrete-time HIV dynamics models with three categories of infected CD4 ${ }^{+}$ T-cells. Adv. Differ. Equ. 2019, 407 (2019)

51. Elaiw, A.M., Alshaikh, M.A.: Global stability of discrete pathogen infection model with humoral immunity and cell-to-cell transmission. Chaos Solitons Fractals 130, 109458 (2020)

52. Bellomo, N., Painter, K.J., Tao, Y., Winkler, M.: Occurrence versus absence of taxis-driven instabilities in a May-Nowak model for virus infection. SIAM J. Appl. Math. 79(5), 1990-2010 (2019)

53. Perelson, A.S. Nelson, PW: Mathematical analysis of HIV-1 dynamics in vivo. SIAM Rev. 41(1), 3-44 (1999)

54. Leenheer, P.D., Smith, H.L.: Virus dynamics: a global analysis. SIAM J. Appl. Math. 63(4), 1313-1327 (2003)

55. Nowak, M.A., Bangham, C.R.M.: Population dynamics of immune responses to persistent viruses. Science 272, 74-79 (1996)

56. Song, X., Neumann, A.: Global stability and periodic solution of the viral dynamics. J. Math. Anal. Appl. 329, 281-297 (2007)

57. Elaiw, A.M., Almalki, S.E., Hobiny, A.D.: Global dynamics of humoral immunity Chikungunya virus with two routes of infection and Holling type-II. J. Math. Comput. Sci. 19(2), 65-73 (2019)

58. Huang, G., Ma, W., Takeuchi, Y.: Global properties for virus dynamics model with Beddington-DeAngelis functional response. Appl. Math. Lett. 22(11), 1690-1693 (2009)

59. Kang, J., Miao, H., Chen, X., Xu, J.B., Huang, D.: Global stability of a diffusive and delayed virus dynamics model with Crowley-Martin incidence function and CTL immune response. Adv. Differ. Equ. 2017, 324 (2017)

60. Adak, D., Bairagi, N.: Bifurcation analysis of a multidelayed HIV model in presence of immune response and understanding of in-host viral dynamics. Math. Methods Appl. Sci. 36, 1-17 (2019). https://doi.org/10.1002/mma.5645

61. Mickens, R.E.: Application of Nonstandard Finite Difference Scheme. World Scientific, Singapore (2000)

62. Mickens, R.E.: Calculation of denominator functions for nonstandard finite difference schemes for differential equations satisfying apositivity condition. Numer. Methods Partial Differ. Equ. 23, 672-691 (2007)

63. Gibelli, L., Elaiw, A., Alghamdi, M.A., Althiabi, A.M.: Heterogeneous population dynamics of active particles: progression, mutations, and selection dynamics. Math. Models Methods Appl. Sci. 27, 617-640 (2017)

64. Elaiw, A.M., AlAgha, A.D.: Global dynamics of reaction-diffusion oncolytic M1 virotherapy with immune response. Appl. Math. Comput. 367, Article ID 124758 (2020)

65. Elaiw, A.M., AlAgha, A.D.: Global dynamics of a general diffusive HBV infection model with capsids and adaptive immune response. Adv. Differ. Equ. 2019, 519 (2019)

66. McCluskey, C.C., Yang, Y.: Global stability of a diffusive virus dynamics model with general incidence function and time delay. Nonlinear Anal., Real World Appl. 25, 64-78 (2015)

67. Shi, P., Dong, L.: Dynamical behaviors of a discrete HIV-1 virus model with bilinear infective rate. Math. Methods Appl. Sci. 37, 2271-2280 (2014)

68. Hattaf, K., Yousfi, N.: Global properties of a discrete viral infection model with general incidence rate. Math. Methods Appl. Sci. 39, 998-1004 (2016)

\section{Submit your manuscript to a SpringerOpen ${ }^{\circ}$ journal and benefit from:}

- Convenient online submission

- Rigorous peer review

- Open access: articles freely available online

- High visibility within the field

- Retaining the copyright to your article

Submit your next manuscript at $\boldsymbol{\triangleright}$ springeropen.com 